\title{
Hitzeinduzierte Radikalbildung während der thermischen Holzmodifizierung
}

\author{
Dissertation \\ zur Erlangung des mathematisch-naturwissenschaftlichen Doktorgrades \\ "Doctor rerum naturalium" \\ der Georg-August-Universität Göttingen \\ im Promotionsprogramm Materialforschung Holz (MaFo Holz) \\ der Georg-August University School of Science (GAUSS) \\ vorgelegt von \\ Nonna Holstein \\ aus Sewastopol
}

Göttingen, 2020 


\section{Betreuungsausschuss}

Prof. Dr. Philipp Vana, MBA Institut für Physikalische Chemie Georg-August-Universität Göttingen

Prof. Dr. Holger Militz

Holzbiologie und Holzprodukte

Georg-August-Universität Göttingen

Prof. Dr. Carsten Mai

Holzbiologie und Holzprodukte

Georg-August-Universität Göttingen

\section{Mitglieder der Prüfungskommission}

\section{Referent}

Prof. Dr. Philipp Vana, MBA Institut für Physikalische Chemie

Georg-August-Universität Göttingen

\section{Korreferent}

Prof. Dr. Carsten Mai Holzbiologie und Holzprodukte

Georg-August-Universität Göttingen

Weitere Mitglieder der Prüfungskommission

Prof. Dr. Holger Militz

Holzbiologie und Holzprodukte

Georg-August-Universität Göttingen

Prof. Dr. Carsten Mai

Holzbiologie und Holzprodukte

Georg-August-Universität Göttingen

Prof. Dr. Gisela Ohms

Naturwissenschaften und Technik

HAWK Göttingen

Jun. Prof. Dr. Kai Zhang Holztechnologie und Holzchemie

Georg-August-Universität Göttingen

apl. Prof. Dr. Thomas Zeuch Institut für Physikalische Chemie

Georg-August-Universität Göttingen

Tag der mündlichen Prüfung: 14. Februar 2020 
Meiner Familie 



\section{Inhaltsverzeichnis}

Abstract 1

Zusammenfassung 3

Einleitung $\quad 5$

$\begin{array}{lll}\text { Kapitel } 1 & \text { Theoretische Grundlagen } & 7\end{array}$

$\begin{array}{lll}1.1 & \text { Struktur der Zellwand } & 7\end{array}$

1.2 Chemie des Holzes 8

1.2.1 Cellulose 8

$\begin{array}{lll}1.2 .2 & \text { Hemicellulosen } & 10\end{array}$

$\begin{array}{ll}1.2 .3 \text { Lignin } & 11\end{array}$

1.2.4 Extraktstoffe 14

1.3 Gewinnung vom Lignin 14

1.4 Thermische Modifikation des Holzes 15

1.5 Chemische Holzveränderungen durch die thermische Modifizierung 16

$\begin{array}{ll}1.6 \text { Radikalische Polymerisation } & 16\end{array}$

Kapitel 2 Thermische Modifizierung des Holzes 19

2.1 Auswirkung der TM auf das Holz 19

2.1.1 Veränderungen auf dem chemischen Niveau im TMT 20

2.1.2 Massenverlust (MS) 30

2.1.3 Wasserkontaktwinkel (WCA) 32

2.2 Stabile freie Radikale im TMT 33

2.2.1 Auswertung von ESR-Spektren 33

2.2.2 Bildung von stabilen freien Radikalen in TMT 35 
2.3 Fazit 40

Kapitel 3 Hitzeinduzierte Strukturänderungen im Lignin 43

3.1 Untersuchte Ligninarten 43

3.2 FT-IR-Spektroskopie 44

3.2.1 IR-Spektroskopie für das technische Lignin 45

3.2.2 IR-Spektroskopie für das extrahierte Lignin 49

3.2.3 IR-Spektroskopie für das synthetisierte Lignin

Modell aus dem Coniferylalkohol 51

3.3 NMR-Spektroskopie $\quad 53$

3.3.1 Unterschiede in den NMR-Spektren zwischen dem Organosolv und dem Indulin 53

3.3.2 NMR Analyse des thermisch behandelten

Indulin Lignins 56

3.3.3 NMR Analyse des thermisch behandelten

Organosolv Lignins $\quad 58$

3.3.4 NMR Analyse des synthetisierten Lignin

Modells aus dem Coniferylalkohol 59

3.4 Gelpermeationschromatographie (GPC) des Lignins 62

3.5 Dynamische Differenzkalorimetrie (DSC) des Lignins 64

3.6 Massenverlust des Lignins nach thermischer Behandlung 66

$\begin{array}{lll}3.7 & \text { Massenspektrometrie (MS) } & 67\end{array}$

3.8 Elektronenspinresonanz Spektroskopie (ESR) des Lignins 68

3.9 Fazit $\quad 72$

Kapitel 4 Behandlung des TMT Pulvers durch eine radikalische Polymerisation 73

4.1 Radikalische Polymerisation des TMT Pulvers in der Substanz ohne Initiator $\quad 73$

$\begin{array}{lll}\text { 4.1.1 TGA Analyse } & 74\end{array}$

$\begin{array}{lll}\text { 4.1.2 ATR-IR Analyse } & 78\end{array}$

4.1.3 Wasserkontaktwinkel (WCA) 82

4.1.4 Dynamische Wasserdampfsorption (DVS) 83

4.2 Radikalische Polymerisation des TMT Pulvers in der Lösung ohne Initiator 86 
4.3 ESR Messungen während der Polymerisationsreaktion 91

4.4 Fazit 94

Kapitel 5 Schlussbemerkungen und Ausblick 97

Kapitel 6 Experimenteller Teil 101

6.1 Geräte und Analysemethoden 101

6.1.1 Gelpermeationschromatographie (GPC) 101

6.1.2 Kernspinresonanz Spektroskopie (NMR) 101

6.1.3 Fourier-Transform-Infrarotspektroskopie (FT-IR) 102

6.1.4 Elektronenspinresonanz Spektroskopie (ESR) 103

6.1.5 Massenspektrometrie (MS) 103

6.1.6 Dynamische Differenzkalorimetrie (DSC) 104

6.1.7 Thermogravimetrische Analyse (TGA) 104

6.1.8 Wasserkontaktwinkel (WCA) 104

6.1.9 Dynamische Wasserdampfsorption (DVS) 104

6.1.10 Thermische Modifikation des Holzes (TMT) 105

6.2 Verwendete Chemikalien und Materialien 106

6.2.1 Holzmaterialien 106

6.2.2 Lignin 106

6.2.3 Monomere 106

6.2.4 Andere Chemikalien 107

6.3 Experimentelle Vorgehensweisen 107

6.3.1 Probenpräparation für TMT 107

6.3.2 Probenpräparation für ESR-Messung 107

6.3.3 Thermische Modifikation des Holzes (TMT) 108

6.3.4 Massenverlust der Holzproben (ML) 108

6.3.5 Bestimmung der chemischen Zusammensetzung $\begin{array}{ll}\text { von TMT } & 109\end{array}$

6.3.6 Synthese eines Lignin Modells aus Coniferylalkohol 110

6.3.7 Ligninreinigung 111

6.3.8 Thermische Behandlung des Lignins und

Bestimmung des Massenverlusts (ML) 111

6.3.9 Behandlung des TMT Pulvers mit Styrol

durch radikalische Polymerisation in der Substanz 112 
6.3.10 Behandlung des TMT Pulvers mit Styrol

durch elementare radikalische Polymerisation im

Lösungsmittel

6.3.11 Behandlung des TMT Pulvers mit NaStS

durch elementare readikalische Polymerisation im

Lösungsmittel

6.3.12 Behandlung des TMT Pulvers mit Styrol

durch elementare radikalische Polymerisation in Substanz während ESR-Messung

Anhang

Abkürzungsverzeichnis

Danksagung

Lebenslauf

Literaturverzeichnis 



\section{Abstract}

In this thesis the hardwood and the softwood were thermally modified to investigate the effect of heat on the chemical structure of the wood and the heat induced radical formation during wood modification. Different analytical methods were used to investigate in the thermally modified timber (TMT).

The dependence of the ration of wood components in the thermally modified wood to the modification parameters was investigated. This change in the structure explains the investigated properties of the wood, such as improved fire resistance or improved hydrophobicity.

The main point of this thesis was the investigation of free stable radicals in the thermally modified wood. It was studied via electron spin resonance (ESR) spectroscopy. It was affirmed that the radical concentration in the thermally modified timber correlates with the modification parameters. The relative humidity $(\mathrm{RH})$, the type of wood and the time after the modification process also influence the radical concentration in TMT.

Since the wood is very complex material, the complexity of the wood made it difficult to consider of the results of the analysis. This was explained by the synergistic degradation reactions of the wood components. Thermogravimetric analysis (TGA) showed the significant differences between the results for the wood and its isolated components. Therefore, the system has been simplified and only lignin studied, as the most radicalcontaining component in TMT.

Three types of lignin were thermally treated - the technical lignin, the extracted lignin from the wood and the synthesized lignin model from the coniferyl alcohol. Therefore, lignin was characterized by a variety of analysis methods. The results showed the heat induced changes in the lignin structure.

The $\mathrm{OCH}_{3}$-groups, the phenolic $\mathrm{OH}$-groups and the $\beta-\mathrm{O}-4$ bonds are broken as the treatment temperature increases.

The presented results confirm the significant differences between the structural changes of hardwood and softwood, also many similar heatinduced decomposition processes in the both type of wood. It was proved that new intramolecular bonds in the structure form under heat. This increases the molecular weight and the degree of crosslinking in TMT. 
The stability of free radicals was explained by the delocalized electron state in the highly conjugated lignin structure ant the limited mobility of the substituents. Despite the increased stability the free radicals can be used profitably by the radical polymerization with styrene. The successful radical polymerization in bulk of the thermally modified wood powder was carried without the initiator. The numerous analytical methods have confirmed that the samples with the higher radical concentration are well suited for this purpose.

The presented results provide an extensive overview of the influence of the thermal modification on changes in the chemical structure of wood and on heat-induced radical formation in the wood. 


\section{Zusammenfassung}

Im Rahmen dieser Arbeit wurde Laub- und Nadelholz thermisch modifiziert, um die Auswirkung der Hitze auf die chemische Struktur des Holzes, genauso wie die hitzeinduzierte Radikalbildung während der Holzmodifizierung mit Hilfe von unterschiedlichen Analysemethoden zu untersuchen.

So wurde gezeigt, dass das Verhältnis der Holzkomponenten im thermisch modifizierten Holz von den Modifikationsparametern abhängig ist.

Diese Änderungen in der Struktur erklären die untersuchten Eigenschaften des Holzes, wie die spätere thermische Zersetzung oder die erhöhte Hydrophobizität.

Der Hauptpunkt dieser Arbeit lag in der Untersuchung der freien stabilen Radikalen im thermisch modifizierten Holz mit Hilfe der Elektronenspinresonanz Spektroskopie (ESR). Dass die Radikalkonzentration im TMT (Thermally Modified Timber) sehr eng mit den Modifikationsparametern korreliert, ${ }^{9}$ konnte bestätigt werden.

Auch die relative Luftfeuchtigkeit, die Holzart und die Zeit nach dem Modifikationsprozess beeinflussen die Radikalkonzentration im TMT.

Die Betrachtung den Analyseergebnissen wurde durch die Komplexität des Holzes erschwert. Dies wurde durch die synergistischen Abbaureaktionen der Holzkomponenten erklärt. ${ }^{58}$ Die thermogravimetrische Analyse (TGA) zeigte den signifikanten Unterschied zwischen den Ergebnissen für das $\mathrm{Holz}$ und seinen isolierten Komponenten. Aus diesem Grund wurde das System vereinfacht und das Lignin, die häufigste radikalenthaltende Komponente im TMT, separat untersucht.

Dafür wurden drei Ligninarten - das technische Lignin, das aus dem Holz extrahirte Lignin und das aus dem Coniferylalkohol synthetisierte Lignin Modell - thermisch behandelt und mit Hilfe der vielfältigen Analysemethoden charakterisiert und die hitzeinduzierten Strukturänderungen gezeigt.

Mit der steigenden Behandlungstemperatur werden die $\mathrm{OCH}_{3}-\mathrm{Gruppen}$ phenolische $\mathrm{OH}-\mathrm{Gruppen}$ und die $\beta-\mathrm{O}-4$-Bindungen in der Struktur stark gebrochen. 
Signifikante Unterschiede zwischen den Strukturänderungen des Laubholzes und des Nadelholzes, aber auch genauso viele ähnliche hitzeinduzierte Zersetzungsprozesse wurden gezeigt und erklärt. So wurde bewiesen, dass sich die neuen intramolekularen Verknüpfungen in der Struktur unter der Hitze bilden. Dadurch werden die Molmasse und der Vernetzungsgrad im TMT vergrößert.

Trotz der höheren Stabilität der freien Radikalen im TMT, die durch den delokalisierten Elektronenzustand in der hoch konjugierten Ligninstruktur und durch die begrenzte Beweglichkeit der Substituenten erklärt wurde, ${ }^{72,73}$ wurde gezeigt, dass sich diese Radikale mittels einer radikalischen Polymerisation mit Styrol gewinnbringend einsetzen lassen. Eine erfolgreiche radikalische Polymerisation des thermisch modifizierten Holzpulvers in der Substanz ohne den Initiator wurde durchgeführt. Die zahlreichen Analysemethoden haben bestätigt, dass die Proben mit der höheren Radikalkonzentration gut dafür geeignet sind.

Die vorgestellten Ergebnisse geben einen umfangreichen Überblick über den Einfluss der thermischen Modifikation auf die Veränderungen der chemischen Struktur des Holzes und auf die hitzeinduzierte Radikalbildung im Holz. 


\section{Einleitung}

Aufgrund von Umweltbedenken besteht ein Bedarf neue Technologien zu entwickeln, bei denen nachwachsende Ressourcen nachhaltig genutzt werden sollen. Das Interesse an der thermischen Holzmodifizierung ist vor Kurzem erneut sehr groß geworden. Diese Prozesse verbessern die Holzeigenschaften und erzeugen ein Material, dass am Ende des Produktionslebenszyklus ohne Schädigungen für die Umwelt entsorgt werden kann. ${ }^{1}$

Die Notwendigkeit, unsere Ressourcen nachhaltig zu nutzen, ist heute besonders bewusst geworden, obwohl das Konzept der Nachhaltigkeit schon längst bekannt ist. Seit über einem Jahrhundert werden die thermisch induzierten verbesserten Eigenschaften des Holzes untersucht. 2,3,6 Trotzdem hat sich die thermische Modifizierung des Holzes industriell und kommerziell erst in den letzten zwanzig Jahren durchgesetzt. Die verbreitetsten Methoden stammen dabei aus Finnland, Frankreich und den Niederlanden. , $^{1,4}$

Das Ziel der thermischen Modifizierung ist die Verbesserung der Eigenschaften des Holzes, wie z. B. die Dimensionsstabilität durch die Verringerung der Wasseraufnahme, die Verbesserung der Witterungsbeständigkeit, genauso wie die höhere Pilzresistenz des Holzes. Das Material wird nur durch die Anwendung von Hitze und ohne chemische und toxische Stoffe hergestellt. $6,7,8$

Die Verbesserung der Eigenschaften des Holzes kann durch seine Komponenten erklärt werden. So führt die thermische Modifizierung des Holzes zu dem Abbau der hydrophilen Hemicellulose. ${ }^{50}$ Dieses macht TMT hydrophober und erhöht z. B. die Dimensionsstabilität. Durch das Verständnis der chemischen Veränderungen im molekularen Niveau können die gewünschten Eigenschaften des Holzes modifiziert werden.

In dieser Arbeit sollen die hitzeinduzierten Veränderungen im molekularen Niveau zwischen dem Laubholz und dem Nadelholz durch unterschiedliche Methoden studiert und deren Abhängigkeit von den Parametern der Modifizierung analysiert werden. Im Mittelpunkt steht die hitzeinduzierte Radikalbildung im TMT (englisch Thermally Modified Timber). Dies wird mit Hilfe von der häufig für die Charakterisierung der freien Radikalen angewendeten Methode untersucht - der Elektronenspinresonanz (ESR) Spektroskopie. 
Die freien stabilen Radikalen, die sich im Holz nach der thermischen Modifizierung bilden, sind von den Modifikationsparametern abhängig. Eine gründliche Untersuchung der Korrelation zwischen der Radikalkonzentration und den Modifikationsparametern kann in der Zukunft für die Bestimmung der Behandlungsqualität vom TMT benutzt werden. ${ }^{9}$

Da das Holz hochmolekular und sehr komplex ist und aus mehreren Komponenten besteht, deren Zersetzungsprozesse sich unter der Hitze gegenseitig beeinflussen, ${ }^{1,8}$ sollte das ganze System vereinfacht werden. Damit können die Zersetzungsmechanismen und die Radikalbildung im TMT leichter interpretiert werden. Deswegen sollten in dieser Arbeit auch die hitzeinduzierten Veränderungen in der Struktur des Lignins untersucht werden. Dafür werden vielfältige Methoden ausgesucht wie z. B. IR-, NMR-, ESR-Spektroskopie, GPC, DSC und viele andere. Auch hier wird das Lignin aus dem Laubholz und dem Nadelholz analysiert.

Durch das Verständnis der bei der thermischen Modifizierung des Holzes auftretenden Radikalreaktionen und des Stabilitätscharakters der Radikalen sollen verschiedene Möglichkeiten untersucht werden, um die freie Radikale im TMT nützlich $\mathrm{zu}$ machen und weiter in einer radikalischen Polymerisation anzuwenden. Daraufhin wird das TMT Pulver mit unterschiedlichen Modifikationsparametern mittels einer radikalischen Polymerisation eingesetzt und mit unterschiedlichen Methoden analysiert 


\section{Kapitel 1 Theoretische Grundlagen}

Seit Jahrtausenden ist Holz ein sehr beliebtes Baumaterial. ${ }^{10}$ Dank modernen Methoden ist es möglich, die Eigenschaften des Holzes durch Veränderungen im molekularen Niveau zu verbessern.

\subsection{Struktur der Zellwand}

In einem mikroskopischen Bereich besteht das Holz aus zahlreichen Zellen. ${ }^{8}$ Die Mittellamelle (CML) verbindet die benachbarten Zellen als Kittsubstanz miteinander und besteht aus Pektin und Lignin. ${ }^{8}$

Die Zellwand einer Holzzelle wird aus Schichten oder Lamellen verschiedener Dicke mit unterschiedlicher Orientierung und unterschiedlichem Mengenanteil von Cellulosefibrillen, Hemicellulose und Lignin aufgebaut. ${ }^{1}$ In der Abb. 1.1 ist eine schematische Darstellung eines Zellwandmodell mit folgenden Schichten gezeichnet: Die Mittellamelle (CML), die Primärwand (P) und die Sekundärwand (S).

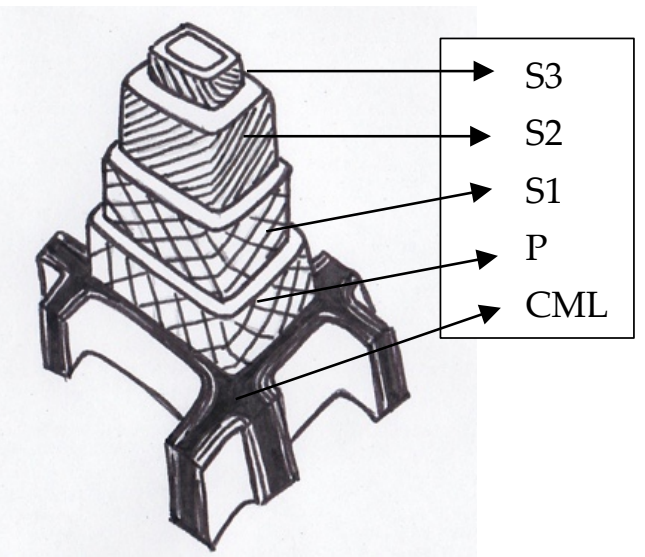

Abb. 1.1: Schematisches Modell einer Zellwand mit der Mikrofibrillenorientierung und den folgenden Schichten: Die Mittellamelle (CML), die Primärwand $(P)$ und die Sekundärwand (S), die aus drei weiteren Schichten S1, S2 und S3 besteht (nachgezeichnet aus C.A.S. Hill). ${ }^{1}$

Die Primärwand ist eine dünne, schwer erkennbare Schicht mit zerstreuter Fibrillenorientierung und mit einem hohen Ligninanteil. ${ }^{8}$ Die sekundäre Schichte hat drei unterschiedlichen Arten der 
Fibrillenordnung mit einem hohen Anteil von Cellulosefibrillen und ist in drei weiteren Schichten unterteilt. Die dünne äußere Sekundärwand (S1) ist eine Übergangslamelle und grenzt an die Primärwand. ${ }^{11}$ Sie enthält Mikrofibrillen, die mit einem Winkel zur Zell-Längsachse von $70^{\circ}$ bis $90^{\circ}$ orientiert sind. ${ }^{11}$ Die zentrale Sekundärwand (S2) ist ein Hauptteil der Zellwand und nimmt das größte Volumen der Zellwand, infolgedessen hat diese Schichte einen großen Einfluss auf die Eigenschaften der Zelle und damit auch auf die Eigenschaften vom Holz. ${ }^{1}$ In dieser Schicht bilden sich Cellulosemikrofibrillen mit dem Winkel zur Längsachse von $10^{\circ}$ bis $30^{\circ} .8$ Die innere Sekundärwand (S3), auch als Tertiärwand genannt, grenzt an den Zellhohlraum an und enthält die Mikrofibrillen mit dem Winkel von $60^{\circ}$ bis $90^{\circ} .8,12$

\subsection{Chemie des Holzes}

Auf einer makroskopischen Ebene ist Holz ein Faserverbundwerkstoff. ${ }^{8}$ Neben Wasser besteht Holz aus vier Hauptbestandteilen. Der größte Anteil gehört zur Cellulose mit $45 \%$ bis 55\%. Danach kommt die Hemicellulose mit $24 \%$ bis $40 \%$. Der drittgrößte Bestandteil ist Lignin mit $18 \%$ bis 35\%. Zum Schluss kommen die Extraktstoffe mit 3\% bis 10\%. ${ }^{13}$ Der Anteil der Komponenten variiert, je nach Baumart und nach Wachstumsbedingungen wie dem Standort des Baums oder dem Klima. Die Zusammensetzung der chemischen Komponenten beeinflusst stark die mechanischen Eigenschaften des Holzes. ${ }^{14}$

\subsubsection{Cellulose}

Eine von den häufigsten organischen Verbindungen, die bei allen Pflanzen das Zellwandgerüst bildet, ist die Cellulose. ${ }^{15}$ Die kleinste sich wiederholende Einheit der Cellulose ist die Cellobiosyl-Einheit, die aus zwei 1,4- $\beta$-D-Glucopyranosen zusammenverknüpft und eine Länge von $1,03 \mathrm{~nm}$ enthält (s. Schema 1.1, oben). ${ }^{16}$ Die Cellobiosyl-Einheiten bilden die Glucanketten, die neben der glycosidischen Hauptvalenzbindungen auch durch intramolekularen Wasserstoffbrücken zusätzlich verknüpft sind (s. Schema 1.1, unten). ${ }^{8}$ Durch intermolekulare Wasserstoffbrücken 
zwischen den Glucanketten bilden sich die Mikrofibrillen, die sich zu Fibrillen zusammenschließen. 8,15,16

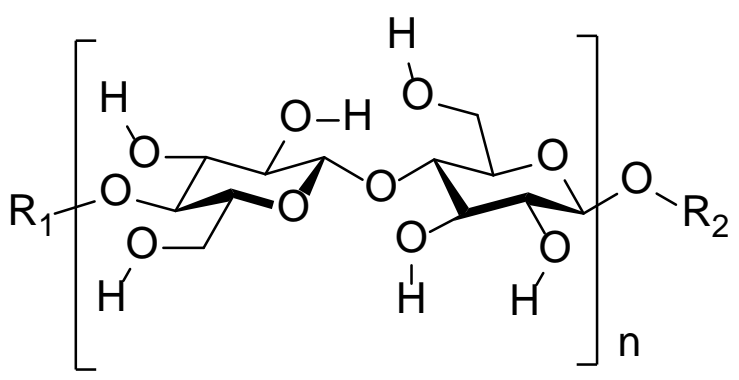

$1,03 \mathrm{~nm}$

intramolekulare

Wasserstoffbrücken

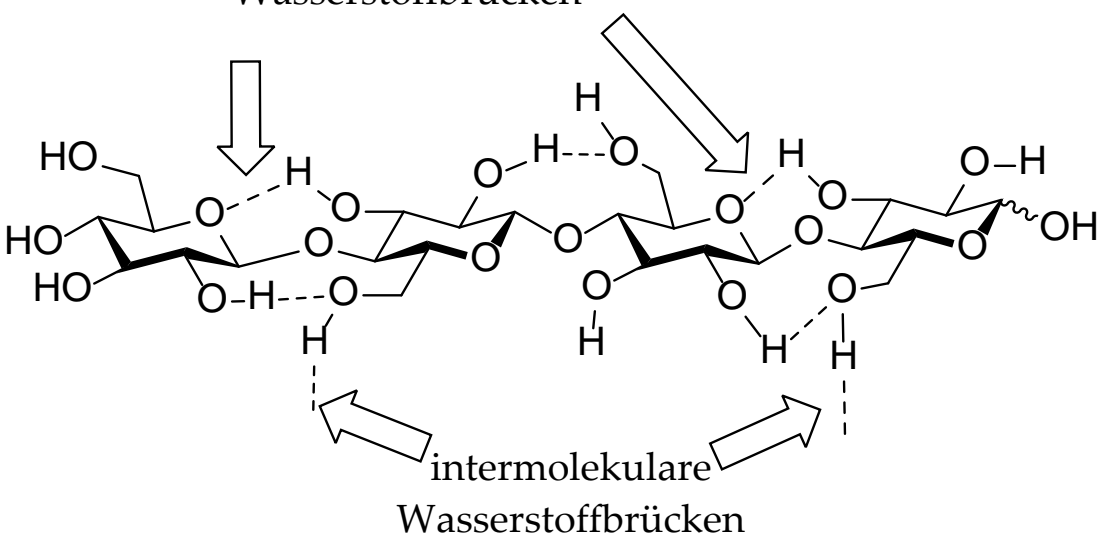

Schema 1.1: Chemische Struktur einer Cellobiosyl-Einheit (oben) und chemische Struktur einer Glucankette mit den intra- und den intermolekularen Wasserstoffbrücken (unten).

Der Polymerisationsgrad (DP) der Cellulose, der die Anzahl der vereinigten Einheiten in der makromolekularen Kette beschreibt, liegt zwischen 300 und 10000 und hängt sowohl von der Herkunft als auch von der Behandlung des Rohmaterials ab. ${ }^{8,17}$

Cellulosen liegen im Holz im Kristallinen und Amorphen Zustand vor. $50 \%$ bis $60 \%$ der Cellulose sind im Kristallinen Bereich. Mit dem Kristallitätsgrad sinkt die Löslichkeit und die Reaktivität der Cellulose. Die Cellulose ist hauptsächlich verantwortlich für die hohe Zugfestigkeit von 120 bis $140 \mathrm{GPa}$, dies ist 10 bis 1000-mal stärker als meisten synthetischen Thermoplasten. ${ }^{10,18,19}$ 


\subsubsection{Hemicellulosen}

Hemicellulosen bestehen aus Polysacchariden mit dem geringen Polymerisationsgrad (DP) von 100 bis 200. Im Gegensatz zur Cellulose bildet die Hemicellulose kurze Kette, diese aus Zucker wie Xylose, Glucose, Mannose, Galactose, Arabinose, Galacturonsäre und Glucuronsäure bestehen (s. Schema 1.2).10,20<smiles>OC(O)C(O)C(O)O</smiles>

$\beta$-D-Xylose

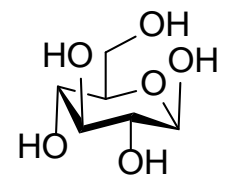

$\beta^{\text {-D-Glucose }}$

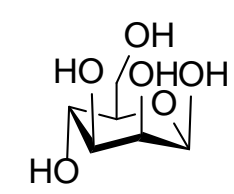

$\beta$-D-Mannose

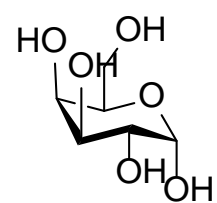

a-D-Galactose

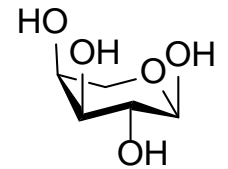

$\alpha_{\text {-L-Arabinopyranose }}$

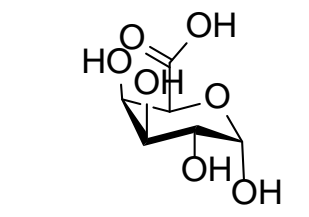

Q-D-Galacturonsäure

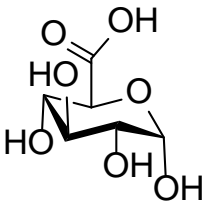

Q-D-Glucuronsäure<smiles>O=C(O)C(O)C(O)C(O)C(O)O</smiles>

$\beta$-D-Glucuronsäure

Schema 1.2: Chemische Strukturen der Monosaccharide.

Die vereinfachten Hauptketten sind Xylane und Glucomannane. Die Xylane sind die lineare, unverzweigte Ketten mit 1-4 glycosidisch gebundenen Anhydroxylosen und Glucomannane sind die lineare Ketten mit 1-4 glycosidisch gebundenen Anhydroglucosen und Anhydromannosen. ${ }^{8}$ Der Unterschied zwischen dem Laubholz und dem Nadelholz liegt an dem unterschiedlichen Verhältnis der Hauptketten und an der unterschiedlichen Verzweigung der Ketten. Das Laubholz enthaltet bis 30\% Xylane und nur 3-5\% unverzweigten Glucomannane mit dem Glucoseanteil zum Mannangehalt 1 zu 1,5. Wohingegen enthaltet Nadelholz nur 10 bis 15\% Xylane und 5 bis 10\% Glucomannane mit dem dreifachen Mannangehalt zum Gluscoseantei. ${ }^{21}$

Hemicellulosen sind verzweigt. Dies führt zu einer amorphen Struktur und erklärt den stark hydroskopischen Charakter, die höhere Reaktivität und geringere thermische Stabilität im Vergleich zur Cellulose oder dem Lignin. ${ }^{8,22}$ Dies ist der Hauptgrund für das Quell- oder Schwindverhalten 
und die Viskoelastizität des Holzes. ${ }^{8}$ Die Cellulosemikrofibrillen werden durch Hemicellulose eingehüllt und somit mit dem Lignin verknüpft. ${ }^{8}$

\subsubsection{Lignin}

Lignin ist ein amorphes, dreidimensionales, hydrophobes Biopolymer und das größte aromatische Makromolekül der Erde. ${ }^{23}$ Lignin wird durch eine enzymatisch initiierte Dehydrierungspolymerisation aus drei MonomerVorstufen aufgebaut. Dies sind Cumarylalkohol (auch als p-Hydroxylphenyl oder weitergehend als H-Lignin Einheit), Coniferylalkohol (auch als Guaiacyl oder G-Lignin Einheit) und Sinapylalkohol (auch als Syringyl oder S-Lignin Einheit), welche im Schema 1.3 dargestellt sind. ${ }^{24}$<smiles>OC/C=C/c1ccc(O)cc1</smiles>

Cumarylalkohol p-Hydroxyphenyl, $\mathrm{H}$<smiles>COc1cc(/C=C/CO)ccc1O</smiles>

Coniferylalkohol Guaiacyl, G<smiles>COc1cc(/C=C/CO)cc(OC)c1O</smiles>

Sinapylalkohol
Syringyl, $\mathrm{S}$

Schema 1.3: Chemische Strukturen der Lignin Einheiten: Cumarylalkohol (H-Lignin Einheit), Coniferylalkohol (G-Lignin Einheit) und Sinapylalkohol (S-Lignin Einheit).

Diese Monomer-Einheiten bestehen aus einem Phenol, einer Propanylkette in der para-Position und einer primären, aliphatischen OH-Gruppe. Lignin Einheiten werden durch unterschiedliche Substituenten definiert (s. Schema 1.3). Allgemein werden Lignine in drei Klassen unterteilt: G-Lignine, GS-Lignine und HGS-Lignine. Die ersten beiden Klassen sind in den Bäumen und die letzte Klasse in Gräsern zu finden. Das Nadelholz hat G-Lignin, der aus 90\% G-Bausteinen besteht und das Laubholz besteht aus GS-Ligninen, wobei der Anteil der S-Einheiten deutlich höher ist als den Anteil der G-Einheiten. Deswegen hat das Laubholz mehr $\mathrm{OCH}_{3}-$ Gruppen als das Nadelholz. Diese blockieren die möglichen reaktiven Zentren, dies zu weniger Quervernetzungen führt. ${ }^{25}$ 
Das Nadelholz enthält mehr Lignin als das Laubholz. ${ }^{8,26}$

Schema 1.4 zeigt, wie die Alkohole enzymatisch zu Phenoxylradikalen oxidiert werden. Diese Radikale werden durch die Mesomerie stabilisiert. ${ }^{27}$

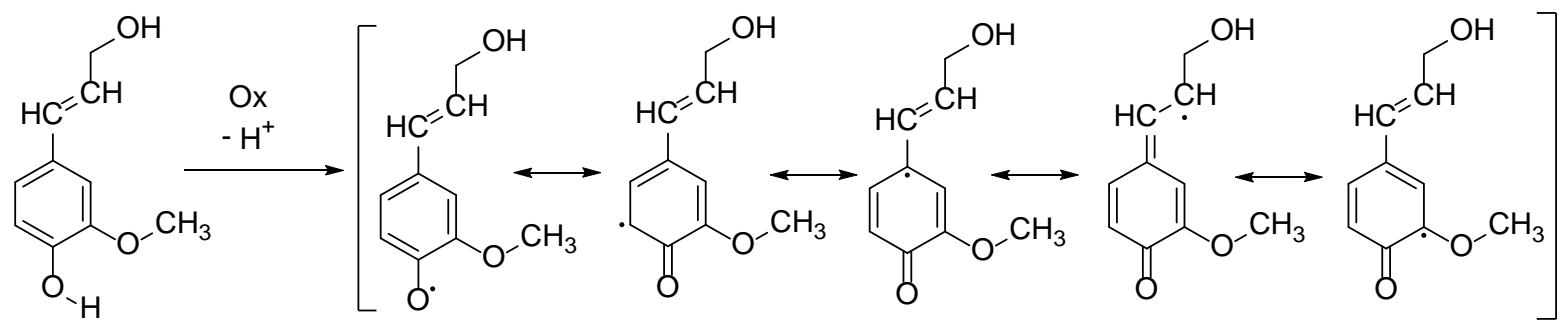

Schema 1.4: Schematische Darstellung die Mesomeriestabilisierung des Phenoxyradikals in der Coniferylalkohols.

Diverse Bindungstypen gibt es zwischen den Monomer-Einheiten eines Lignins. Im Schema 1.5 sind einige davon dargestellt.

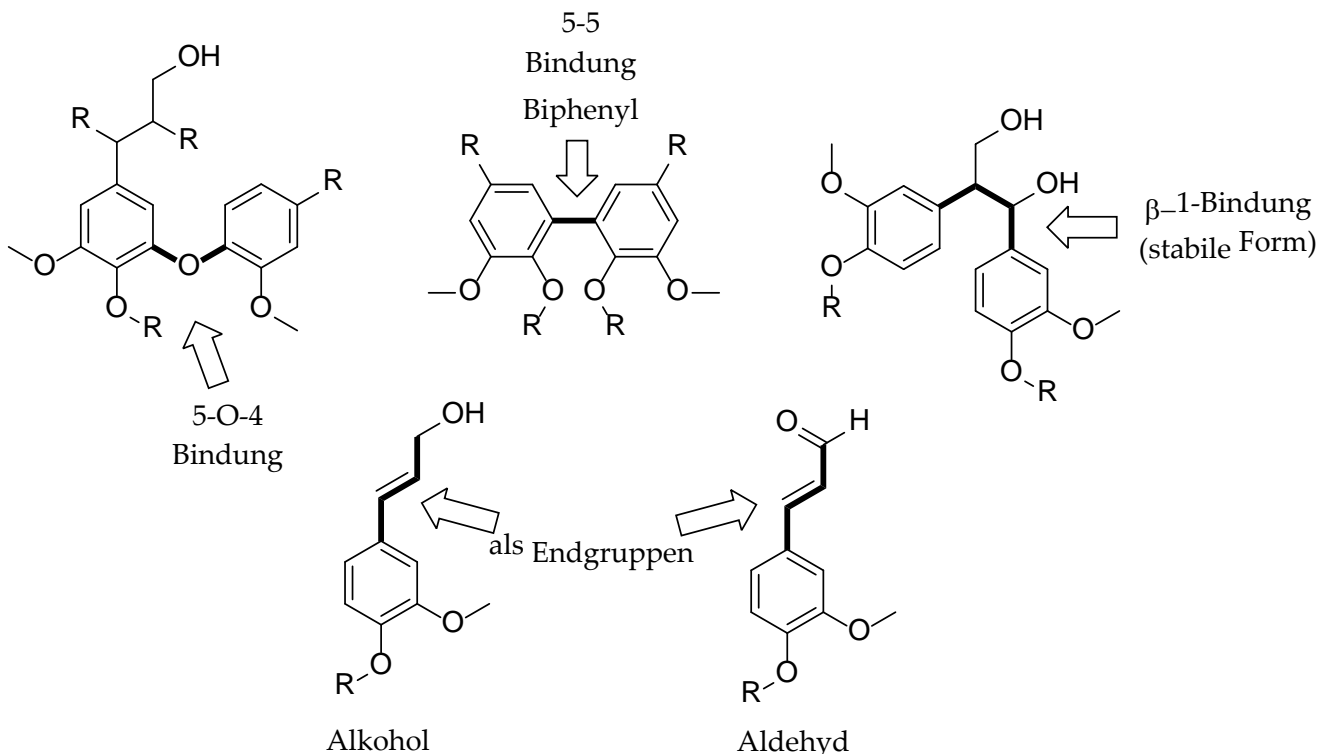

Schema 1.5: Schematische Darstellung von den Verknüpfungen in Lignin.

Die häufigste Bindung im Lignin ist die $\beta-\mathrm{O}-4-$ Bindung (auch als $\beta$-Arylether Bindung genannt). Die chemische Struktur einer $\beta-\mathrm{O}-4-$ Bindung eines Lignins ist im Schema 1.6 dargestellt. 


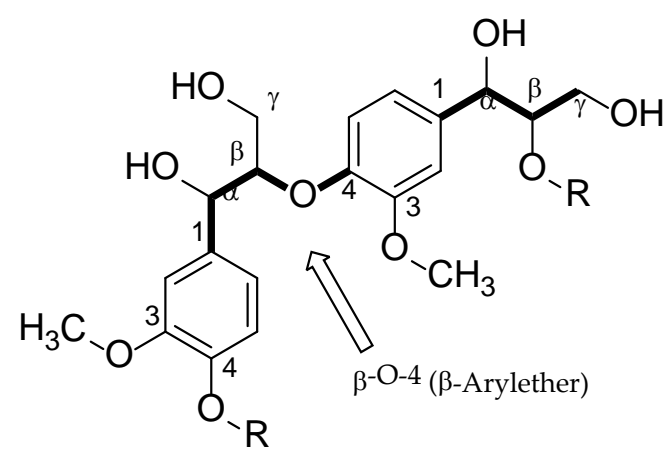

Schema 1.6: Schematische Darstellung $\beta$-O-4 Bindung.

Bis zu $60 \%$ der Monomeren eines G-Lignins sind durch diese Bindung miteinander verknüpft. Eine größere Variationsbreite bieten die GS-Lignine. ${ }^{8}$ Deswegen hat das Ligninmakromalekül eine sehr komplexe Struktur. So zeigt das Schema 1.7 einen kleinen Ausschnitt einer Ligninstruktur aus dem Nadelholz. ${ }^{28}$<smiles>[R]OC(COc1ccc(C(O)C(CO)Oc2ccc([R])cc2OC)cc1)C(O)c1cc(OC)c(OC(CO)C(O)c2ccc(OC(CO)C(O)c3cc(OC)c4c(c3)-c3cc(C(O)C(CO)Oc5ccc(/C=C/CO)cc5OC)cc(OC)c3OC(c3ccc(O)c(OC)c3)C(CO)O4)c(Oc3ccc(C(O)C(CO)Oc4ccc(C5Oc6c(OC)cc(C(O)C(O)CO)cc6C5CO)cc4OC)cc3)c2)c(OC)c1</smiles>

Schema 1.7: Ausschnitt des Lignins aus dem Nadelholz. ${ }^{28}$ 


\subsubsection{Extraktstoffe}

Nächte wichtiger Hauptbestandteil des Holzes sind die Extraktstoffe. Dazu gehören die organischen und anorganischen Verbindungen: Wachse, Fette, Phenole, Isopreonide und Mineralstoffe, wie z. B. Calcium oder Magnesium, die innerhalb der Hohlräume in den Zelllumina eingelagert sind. ${ }^{8}$ Manche Inhaltsstoffe befinden sich in speziellen Harz- und LatexGängen.

Die physiologisch aktive Stoffwechselzwischenproddukte wie Fette, Zucker oder Stärke sind ein Teil von den primären Inhaltsstoffen. Die sekundären Inhaltsstoffe werden während der premortalen Biosynthese als Endprodukte freigesetzt und funktionieren als eine Abwehrt von Mikroorganismen. ${ }^{8,29}$

\subsection{Gewinnung vom Lignin}

Jährlich fallen etwa 50 Millionen Tonnen industrielles Lignin in Form von Ablaugen beim Aufschluss vom Holz. ${ }^{30}$ Davon werden nicht mehr als 2\% als Grundlage für die weitere Produktion verwendet und der Großteil zur Wärmegewinnung oder, um die anorganischen Aufschlusschemikalien zurückzugewinnen, verbrannt. ${ }^{31,32}$ Lignin wird in der Produktion von Klebern, Agrochemikalien oder thermoplastischen Kunststoffen verwendet. ${ }^{33,34}$

In Abhängigkeit des Gewinnungsverfahrens werden unterschiedlichen Ligninarten erzeugt.

Häufig industriel verwendende Verfahren um Lignin zu gewinnen sind der Kraft-Prozess (auch als Sulfatverfahren genannt) und Sulfitverfahren. Der Großteil des Lignins wird mit dem alkalischen Aufschluss dargestellt. ${ }^{35}$ Das Holzmaterial wird mit Natronlauge und Natriumsulfid mehrere Stunden unter dem Druck bearbeitet. Das Sulfid Anion greift an das Lignin an. Dabei wird Lignin gespaltet. ${ }^{24}$

Organosolv Prozess wird auch verwendet, um das Lignin zu gewinnen. Dabei wird ein organisches Lösungsmittel, z. B. Aceton, Methanol, Ethanol oder Essigsäure, bei der höheren Temperaturen zwischen $140{ }^{\circ} \mathrm{C}$ und 180 ${ }^{\circ} \mathrm{C}$ eingesetzt, um das Lignin zu lösen. Dank niedrigen Siedetemperaturen der verwendeten Lösungsmittel wird Lignin leicht 
zurückgewonnen. ${ }^{36}$ Nachteil beiden Verfahren für diese Arbeit liegt daran, dass die $\beta-\mathrm{O}-4$-Bindungen im Lignin durch diese Prozesse gebrochen werden. ${ }^{37}$ Dabei wird oft das Laubholz im Organosolv Prozess verwendet, weil die S-Lignin-Einheiten weniger Quervernetzungen ausbilden. Dies führt zu weniger Sekundärreaktionen während der Produktion. ${ }^{8}$

\subsection{Thermische Modifikation des Holzes}

Eine thermische Modifikation des Holzes ist eine effektive Methode, um die Eigenschaften des Holzes zu verbessern. ${ }^{41}$ Dank ihrer nicht toxischen und dadurch umweltfreundlichen Prozesse weckt die thermische Modifikation großes Interesse in der Forschungs- und in der Industriewelt.

Die Haupteffekte, die durch die thermische Modifikation erreicht werden, sind die Verbesserung der Dimensionsstabilität des Holzes, die Reduktion der Hygroskopizität, die Erhöhung des Widerstandes gegen mikrobiologische Angriffe, jedoch mit Abnahme der mechanischen Festigkeit und die gleichmäßige Verdunklung des Materials. ${ }^{38,39,40,41}$

Die Eigenschaften vom thermisch modifizierten Holz hängen stark von den Behandlungsparametern $a b .{ }^{58}$ Verwendete Behandlungs-temperaturen liegen zwischen $160{ }^{\circ} \mathrm{C}$ und $240{ }^{\circ} \mathrm{C}$. Die unterschiedliche Betriebsatmosphären, wie z. B Dampf, Vakuum oder Stickstoff, ist möglich $\mathrm{zu}$ variieren. Die Modifikation wird entweder im Reaktor mit einem geschlossenen System mit Druck oder einem offenen System ohne Druck durchgeführt. Zeit der Behandlung gehört ebenfalls zu den wichtigen Prozessvariablen. ${ }^{42,43}$ Durch die vielfältigen kontrollierten Behandlungsparametern werden gewünschte Eigenschaften des Holzes erreicht.

In den letzten Jahrzehnten wurden viele thermische Modifizierungsmethoden entwickelt. Häufig verwendete kommerzielle Prozesse sind ThermoWood ${ }^{\circledR},{ }^{44}$ FirmoLin $^{\circledR},{ }^{45}$ PLATO $^{\circledR}$ Wood, ${ }^{46}$ Wood Treatment Technology (WTT) ${ }^{47}$ und TERMOVUOTO. ${ }^{48}$

Die Jahresproduktion in Europa liegt ca. bei $400000 \mathrm{~m}^{3} .{ }^{49}$ Auf Grund seiner Eigenschaften wie höhere Pilzresistenz oder die Dimensionsstabilität wird thermisch modifiziertes Holz oft für Holz in der Außeranwendung, wie 
Terrassen, Fassaden oder Gartenmöbeln verwendet.

\subsection{Chemische Holzveränderungen durch die thermische Modifizierung}

Eigenschaften des thermisch modifizierten Holzes werden mit Hilfe von chemischen Veränderungen nach der Wärmebehandlung erklärt. Das Erhitzen vom Holz führt zu den Abbauprozessen auf dem molekularen Niveau. Die signifikanten Veränderungen in der chemischen Zusammensetzung des Holzes können schon ab $120^{\circ} \mathrm{C}$ quantifiziert werden. ${ }^{56}$

Bei der thermischen Behandlung wird die Hemicellulose schon bei der relativ niedrigen Temperatur stark abgebaut, wobei Essigsäure, Ameisensäure, Methan und Kohlenstoffdioxid abgespalten werden. ${ }^{50}$ So wird die Pilzresistenz und die Dimensionsstabilität verbessert, weil die Hemicellulose sehr hydrophil ist. ${ }^{8}$ Die Essigsäure wirkt weiter als ein Katalysator, beschleunigt die weiteren Abbauprozessen und bildet Furfural, Formaldehyd und andere Aldehyde. ${ }^{60}$

Danach folgen die Zersetzungsprozesse des amorphen Bereichs der Cellulose. Die Cellulose wird weniger beeinflusst durch die Hitze als Hemicellulose und die Abbauprozesse dauern länger. ${ }^{60}$

Schließlich wird die thermisch stabilste Komponente Lignin durch hydrolytische Spaltung, Oxidationsreaktion abgebaut und durch Kondensationsreaktion stark verändert. ${ }^{8}$ Dies führt $\mathrm{zu}$ dem Festigkeitsverlust, dem Massenverlust sowie zu der Verdunkelung des Holzes. 51,52

\subsection{Radikalische Polymerisation}

Eine radikalische Polymerisation ist eine Kettenreaktion, die aus drei Hauptschritten besteht: Initiierungs-, Wachstums- und Abbruchschritt. Reaktionsgleichungen der radikalischen Polymerisation sind im Schema 1.8 dargestellt. 
Startreaktion (a) radikalischer Polymerisation ist die Erzeugung von Initiator-Radikalen. Da die Spaltung der Monomeren in Radikale bei sehr hohen Temperaturen oder durch kurzwellige Strahlung ( $\gamma$-Strahlen) möglich ist, wird die Polymerisationsreaktion meist durch Zugabe von Initiatoren mit deutlich milden Bedingungen (UV-Licht, $<100^{\circ} \mathrm{C}$ ) gestartet. ${ }^{53}$ Wichtige Starter sind Diacylperoxide und Azo-Verbindungen. Zum Starten der Reaktion wird der Initiator in der Substanz oder dem gelösten Monomer eingesetzt und der Prozess wird photochemisch oder thermisch in Gang gebracht.

Dies führt zur Bildung vom Radikal am Initiator (I) und weitere Polymerisation Initiierung durch eine Addition (b) mit einem Monomer (M) an die reaktive Doppelbindung.

In dem nächsten Propagation Schritt (c) erzeugt die Addition des Folgeradikals an ein weiteres Monomer ein dimeres Radikal. ${ }^{49}$ Die Kette $(\mathrm{R} \bullet$ ) wächst mit einem anderen Radikal am Kettenende.

Zum Schluss folgt die Terminierung (d), die durch eine Kombination oder eine Disproportionierung zum Polymer (P) erfolgt. Die Kombination ist die Verknüpfung zweier aktiver Oligo- oder Makroradikale zu einem inerten Polymer. ${ }^{49}$ Bei der Disproportionierung entstehen ein gesättigtes und ein ungesättigtes Polymere.
(a) Initiator

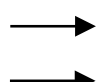
$2 \mathrm{I} \bullet$
(b) $\mathrm{I} \bullet+M$
$\mathrm{R}_{1} \bullet$
(c) $\mathrm{R}_{\mathrm{i}} \bullet+\mathrm{M}$
$\mathrm{R}_{\mathrm{i}+1} \bullet$
(d) $R_{\mathrm{i}} \bullet+R_{\mathrm{j}} \bullet \longrightarrow$
$P_{i+j}$
$\mathrm{R}_{\mathrm{i}} \bullet+\mathrm{R}_{\mathrm{j}} \bullet \longrightarrow$
$P_{i}+P_{j}$

Schema 1.8: Reaktionsgleichungen einer radikalischen Polymerisation mit dem Initiierungsschritt (a), dem Kettenstart (b), der Propagation (c) und der Terminierung (d) durch eine Kombination oder Disproportionierung. 



\section{Kapitel 2 Thermische Modifizierung des Holzes}

Seit Langem wird die thermische Modifikation angewendet, um die Eigenschaften des Holzes zu verbessern. Tiemann ${ }^{54}$ war einer von den Ersten, der vor hundert Jahren über die Wirkung der Hochtemperaturbehandlung auf die physikalischen Eigenschaften von Holz berichtet hat. Industriell wird die thermische Modifikation aber erst seit Ende des zwanzigsten Jahrhunderts genutzt und macht seither große Fortschritte in Europa und USA.43,44 Sehr viele Fragen in den Modifikationsprozessen bleiben aber immer noch offen. Deswegen ist es notwendig, diese Prozesse auch weiter zu untersuchen, um die Methode zu verbessern.

Kapitel 2 befasst sich mit Laboruntersuchungen und den Ergebnissen zur thermischen Modifikation (TM) des Nadelholzes (Kiefer) und Laubholzes (Buche). Alle Prüfkörper werden zuerst in der Fakultät für Forstwissenschaften und Waldökologie in einem Behandlungsreaktor mittels eines offenen Systems beim Atmosphärendruck und einem geschlossenen System mit dem Druck von 5·105 Pa thermisch modifiziert. Genauer sind die Modifikationsprozesse in dem Abs. 6.3 .3 beschrieben. Die Modifikationstemperatur liegt zwischen $180^{\circ} \mathrm{C}$ und $220^{\circ} \mathrm{C}$. In diesem Bereich sind die Veränderungen auf dem makromolekularen Niveau deutlich zu sehen.

\subsection{Auswirkung der TM auf das Holz}

Aufgrund thermisch induzierter chemischer Veränderungen der makromolekularen Komponenten des Holzes werden die physikalischen und biologischen Eigenschaften verändert.50 Über die verbesserten physikalischen Eigenschaften wurde im Kapitel 1 eingegangen.

In dieser Arbeit sind die Veränderungen zwischen dem Laubholz und dem Nadelholz nach der thermischen Behandlung studiert und dessen Abhängigkeit von den Parametern der Modifikation analysiert.

\subsubsection{Veränderungen auf dem chemischen Niveau im TMT}

Wenn das Holz bei höheren Temperaturen behandelt wird, treten die chemischen Änderungen innerhalb der makromolekularen Struktur ein. Eine thermische Zersetzung des Holzes kann schon unter $100{ }^{\circ} \mathrm{C}$ 
beginnen. ${ }^{55} \mathrm{Ab}$ einer kritischen Temperatur von $120{ }^{\circ} \mathrm{C}$ haben Kollmann et al. erste Veränderungen in der chemischen Zusammensetzung des Holzes quantifiziert. ${ }^{56}$ Die signifikanten Veränderungen liegen bei deutlich höheren Temperaturen ab $230{ }^{\circ} \mathrm{C}$. Einige Studien sind bekannt, in denen die chemischen Veränderungen im Holz während der thermischen Behandlung durch Isolierung der Holzbestandteile untersucht wurden. ${ }^{58}$ Die Ergebnisse sind recht variabel und stark abhängig von den Methoden der Herstellung der Prüfkörper sowie von den experimentellen Parametern. ${ }^{57}$

Die thermogravimetrische Analyse (TGA) ist eine hochpräzise Methode, um die Masseänderung der Prüfkörper in Abhängigkeit von der Temperatur und der Zeit $\mathrm{zu}$ bestimmen und ist für das thermisch modifizierte Holz gut geeignet. Die Masseänderungen werden als eine Temperatur- oder auch als eine Zeitfunktion auf einem Thermogramm (TG) aufgetragen, wobei jeder Masseverlust als eine TG-Stufe gezeichnet wird. Dank erster Ableitung des Messsignals (dTG) erscheinen alle TG-Stufen als separate Peaks. So werden auch kleine und schlecht aufgelöste TG-Stufen besser erkannt. Die Thermogramme und ihre Ableitungen für thermisch modifizierte Buche bzw. Kiefer (mit den Modifikationsparametern $T_{\bmod } 180^{\circ} \mathrm{C}, 200^{\circ} \mathrm{C}, 220^{\circ} \mathrm{C}$, nicht modifizierte Probe als Referenz und $p 5$ bar) sind in den Abb. 2.3-2.4 zusammengefasst.

Ergebnisse von isolierten Holzbestandteilen, wie Lignin, Cellulose und Hemicellulose, (Abb. 2.1-2.2) unterscheiden sich stark von den Ergebnissen des Holzes (Abb. 2.3-2.4). Dieses wird durch synergistische Abbaureaktionen der Komponenten erklärt. Unter Temperaturerhöhung starten konkurrierende Prozesse, die zu endothermen und exothermen Ereignissen gleichzeitig führen. ${ }^{58}$

Wie es in den Abb. 2.1-2.2 gezeigt ist, beginnt das Lignin (sowohl Organosolv als auch Indulin) als Erstes seine Masse zu verlieren. Diese Prozesse sind aber sehr langsam, so dass die signifikante Massenänderungen erst ab $260{ }^{\circ} \mathrm{C}$ zu sehen ist. Dafür verliert $\mathrm{D}(+)-X y l o s e$, als Baustein von Hemicellulose, den größten Massenanteil als Erste und deutlich schneller als Lignin. Abbauprozesse starten schon 


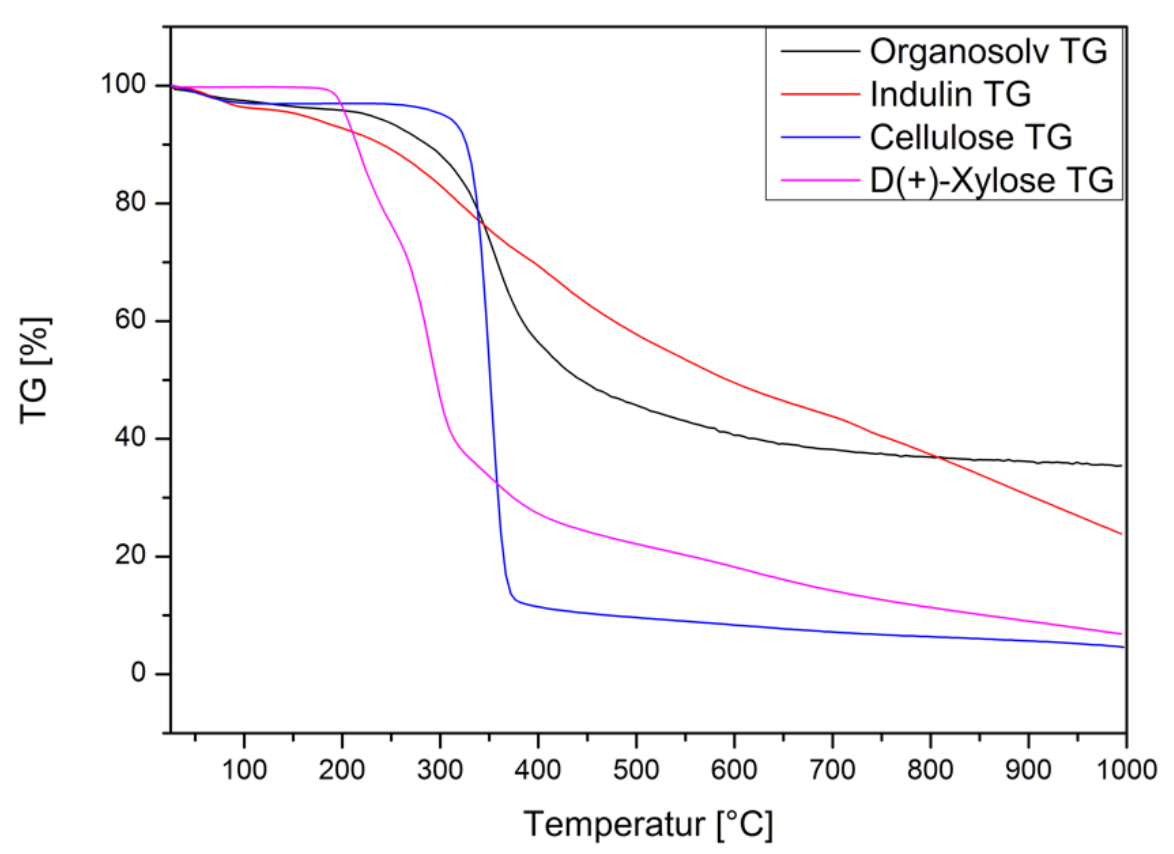

Abb. 2.1: Thermogramm für isolierte Bestandteile des Holzes: Organosolv (schwarz), Indulin (rot), Cellulose (blau) und $\mathrm{D}(+)-X y l o s e ~(l i l a)$.

ab ca. $165^{\circ} \mathrm{C}$. Der erste Peak des Massenverlusts liegt bei $217^{\circ} \mathrm{C}$ und der schnellste Massenverlust ist beim zweiten Peak bei $292^{\circ} \mathrm{C}$ (s. Abb. 2.2).

Während der Abbauprozesse der Hemicellulose werden Essigsäure, Ameisensäure, Methanol und nicht kondensierbare Gase (hauptsächlich $\mathrm{CO}_{2}$ ) freigesetzt. ${ }^{59}$ Die Essigsäure dient weiter als Katalysator und beschleunigt die Bildung von Furfural, Formaldehyde und andere Aldehyde. ${ }^{60}$

Cellulose ist im niedrigen Temperaturbereich bis ca. $250^{\circ} \mathrm{C}$ relativ stabil, verliert ihre Masse aber zwischen $270{ }^{\circ} \mathrm{C}$ und $390^{\circ} \mathrm{C}$ schlagartig.

Auf jedem Thermogramm für thermisch modifizierte Buche bzw. Kiefer (s. Abb. 2.3-2.4) ist nur eine TG-Stufe zu erkennen. Der Massenverlust liegt zwischen $230{ }^{\circ} \mathrm{C}$ und $450{ }^{\circ} \mathrm{C}$. Und in dem Temperaturbereich bei ca. $100{ }^{\circ} \mathrm{C}$ befindet sich ein kleiner nicht relevanter Massenverlust von Wasser unter $2 \%$. 


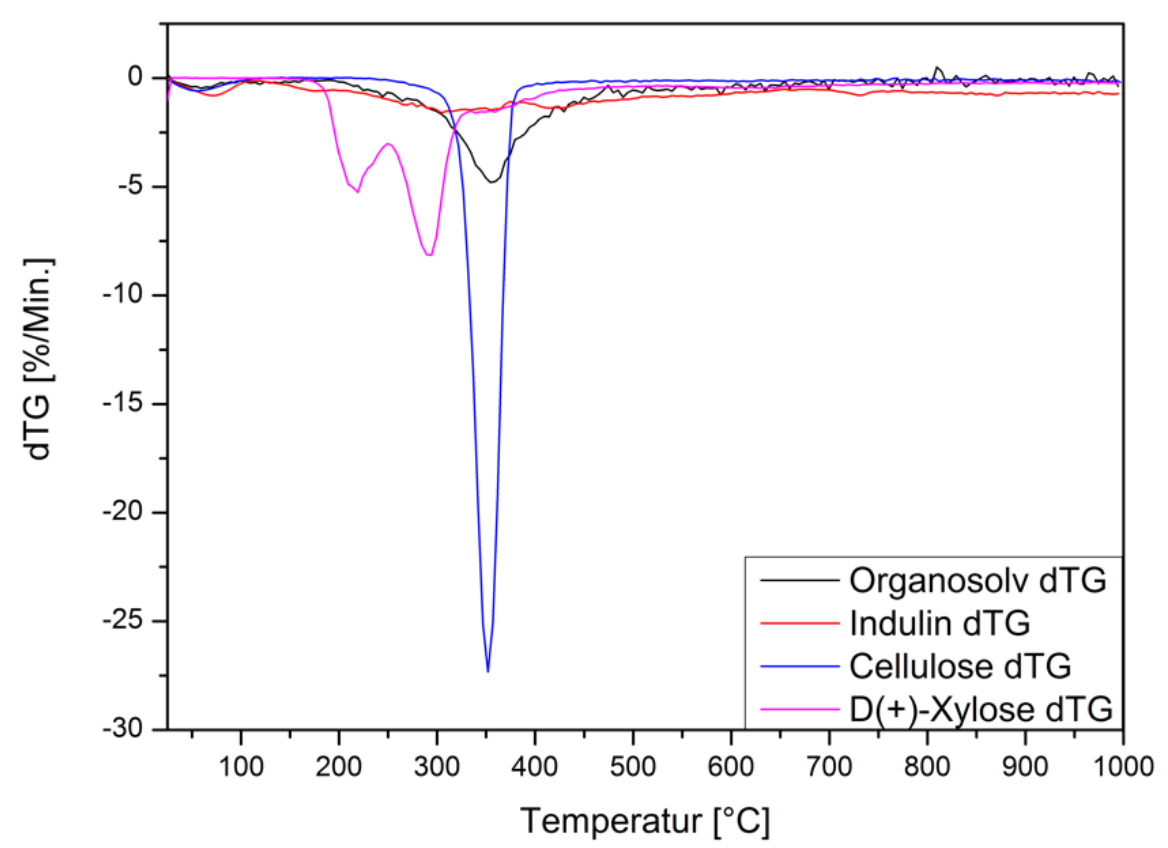

Abb. 2.2: Erste Ableitung vom Thermogramm für isolierte Bestandteile des Holzes: Organosolv (schwarz), Indulin (rot), Cellulose (blau) und D(+)-Xylose (lila).

Thermisch modifiziertes Holz baut sich später bei höheren Temperaturen $\mathrm{ab}$ als nicht modifiziertes Holz (s. Tab. 2.1). Beispielsweise ist der Massenverlust von $5 \%$ für die Referenz aus der Buche bei $260{ }^{\circ} \mathrm{C}$ und für die thermisch modifizierte Buche mit $T_{\bmod }=220^{\circ} \mathrm{C}$ (B220) bei $290^{\circ} \mathrm{C}$ (s. Abb. 2.3 oben). Des Weiteren wird modifiziertes Holz langsamer abgebaut und die Zersetzungstemperaturdifferenz zwischen Referenzprobe und modifizierte Probe wird nur größer. Demnach startet die thermische Zersetzung des thermisch modifizierten Holzes später als bei dem nicht modifizierten Holz.

In den Abb. 2.3 - 2.4 sind die Unterschiede im thermischen Abbauverhalten zwischen dem Laub- und dem Nadelholz bzw. zwischen der Buche und der Kiefer zu erkennen. Die Referenzprobe aus der Buche beginnt sich fast $10^{\circ} \mathrm{C}$ früher zu zersetzen als die Referenzprobe aus der Kiefer. Dennoch dauern die Zersetzungsprozesse in der thermisch modifizierten Buche länger als in der thermisch modifizierten Kiefer. 

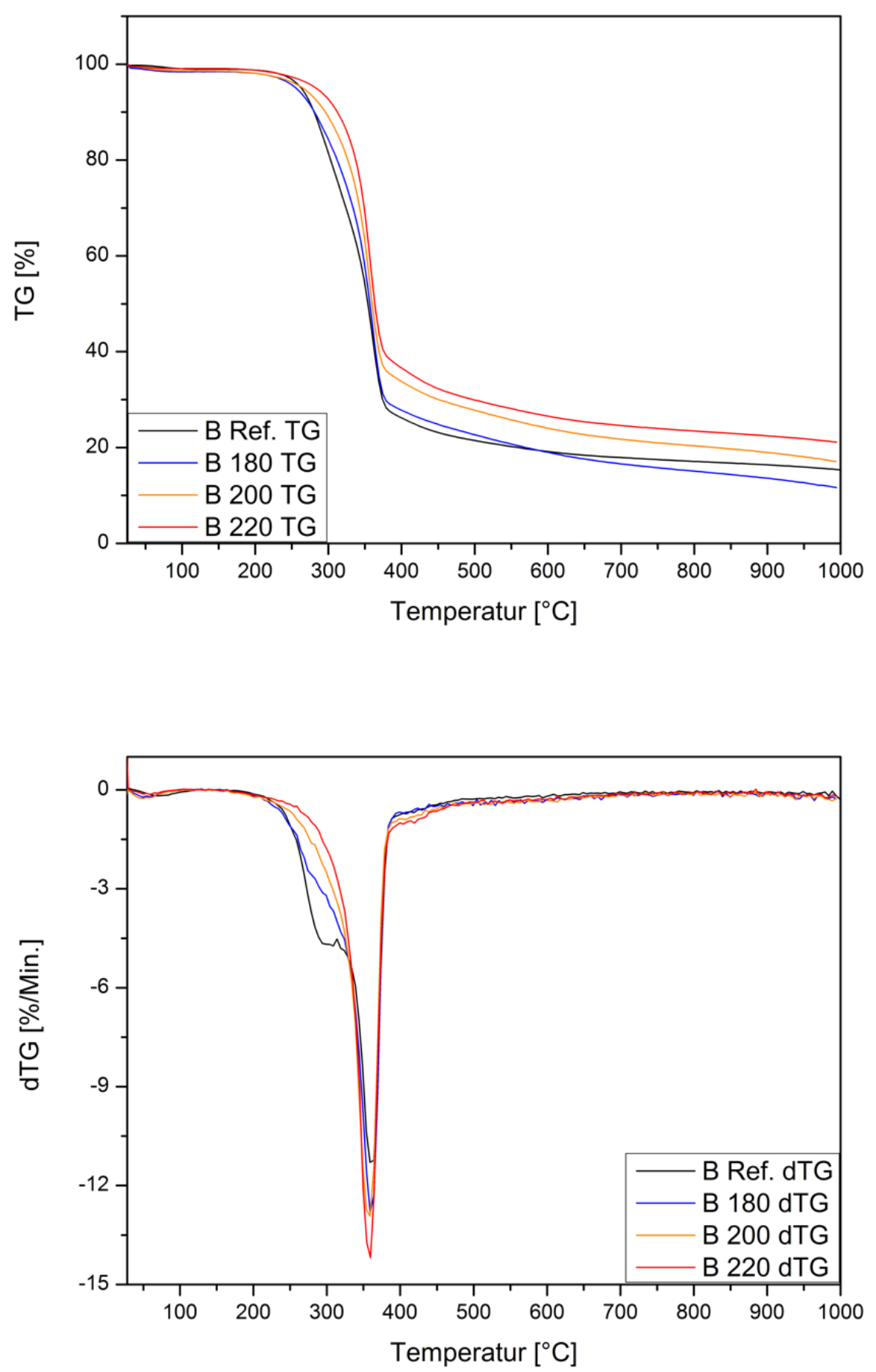

Abb. 2.3: Thermogramm (oben) und die erste Ableitung des Thermogramms (unten) für die Referenz Buche (schwarz) und die thermisch modifizierte Buche unter folgenden Modifikationsparametern: Druck 5 bar und Modifikationstemperaturen $180{ }^{\circ} \mathrm{C}$ (blau), $200{ }^{\circ} \mathrm{C}$ (orange) sowie $220^{\circ} \mathrm{C}$ (rot). 

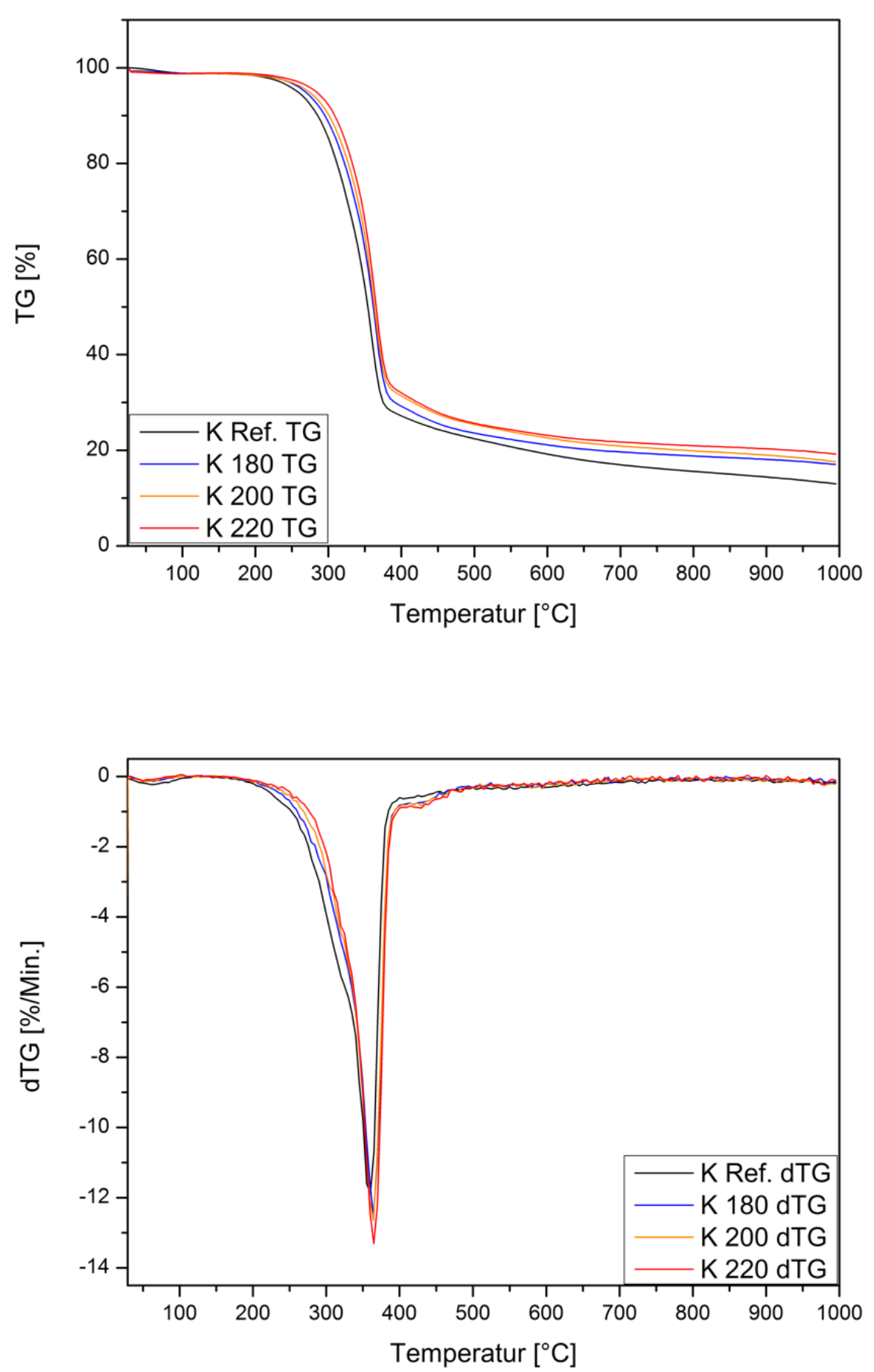

Abb. 2.4: Thermogramm (oben) und die erste Ableitung vom Thermogramm (unten) für die Referenz Kiefer (schwarz) und die thermisch modifizierte Kiefer mit den folgenden Modifikationsparametern: Druck 5 bar; Modifikationstemperaturen $180{ }^{\circ} \mathrm{C}$ (blau), $200{ }^{\circ} \mathrm{C}$ (orange) sowie $220^{\circ} \mathrm{C}$ (rot). 
Tab. 2.1: Anfangszersetzungstemperaturen ( $\left.T_{\text {Anf }}\right)$ und maximale schnellste Abbautemperaturen $\left(T_{\max }\right)$ von der unbehandelten Buche bzw. der Kiefer (hier als Referenz Probe bezeichnet) und von der thermisch modifizierten Buche bzw. der Kiefer mit den Modifikationstemperaturen von $180^{\circ} \mathrm{C}, 200^{\circ} \mathrm{C}$ und $220^{\circ} \mathrm{C}$ und dem Modifikationsdruck von 5 bar.

\begin{tabular}{|c|c|c|}
\hline Probe & $\boldsymbol{T}_{\max }\left[{ }^{\circ} \mathbf{C}\right]$ & $\boldsymbol{T}_{\text {Anf }}\left[{ }^{\circ} \mathbf{C}\right]$ \\
\hline Buche Referenz & 363 & 230 \\
\hline Buche $180^{\circ} \mathrm{C}$ & 360 & 230 \\
\hline Buche $200^{\circ} \mathrm{C}$ & 358 & 254 \\
\hline Buche $220^{\circ} \mathrm{C}$ & 358 & 285 \\
\hline Kiefer Referenz & 360 & 239 \\
\hline Kiefer $180{ }^{\circ} \mathrm{C}$ & 364 & 246 \\
\hline Kiefer $20{ }^{\circ} \mathrm{C}$ & 364 & 256 \\
\hline Kiefer $220^{\circ} \mathrm{C}$ & 365 & 272 \\
\hline
\end{tabular}

Diese Änderungen können durch ein Verhältnis der Komponenten im Holz und ihren Zersetzungen während der thermischen Modifikation erklärt werden. Es ist bekannt, dass die Cellulose mit ca. $45 \%$ den größten Anteil im nicht modifizierten Holz hat; die Hemicellulose hat zwischen $20 \%$ und $40 \%$; der Lignin-Anteil liegt zwischen $18 \%$ und $35 \%$ und die Extraktstoffe haben die niedrigsten Werte von 2\% bis 10\%. Der Anteil der Holzkomponenten ist von der Baumart, dem Klima und dem Ort im Stamm des Baumes abhängig. ${ }^{.1,62}$

In dieser Arbeit wird aber das Verhältnis der Holzkomponenten nach der thermischen Modifikation untersucht. Die Methode dafür ist im Abs. 6.3.5 beschrieben. Die verwendete Methode war ursprünglich für nicht modifiziertes Holz beabsichtigt, aber, wie Experimente ${ }^{113}$ von Wentzel et al. gezeigt haben, dieses Verfahren auch für thermisch modifiziertes Holz funktioniert. Als Modifikationsparameter werden $T_{\bmod } 180^{\circ} \mathrm{C}, 200{ }^{\circ} \mathrm{C}$ und $220^{\circ} \mathrm{C}$ und ein geschlossener Prozess mit Druck von 5 bar verwendet. Alle Werte des modifizierten Holzes sind mit den Werten der Referenzproben verglichen und in der Abb. 2.5 zusammengefasst. Wie erwartet, liegt der Extraktstoff-Anteil unter 5\%. In der Abb. 2.5 (oben) ist $\mathrm{zu}$ erkennen, dass der Lignin-Anteil mit der höheren Modifikationstemperatur steigt. Wie bereits oben gezeigt ist (s. Abb. 2.1), wird das Lignin deutlich langsamer abgebaut als die Hemicellulose oder die Cellulose. Deswegen steigt der Lignin-Anteil 

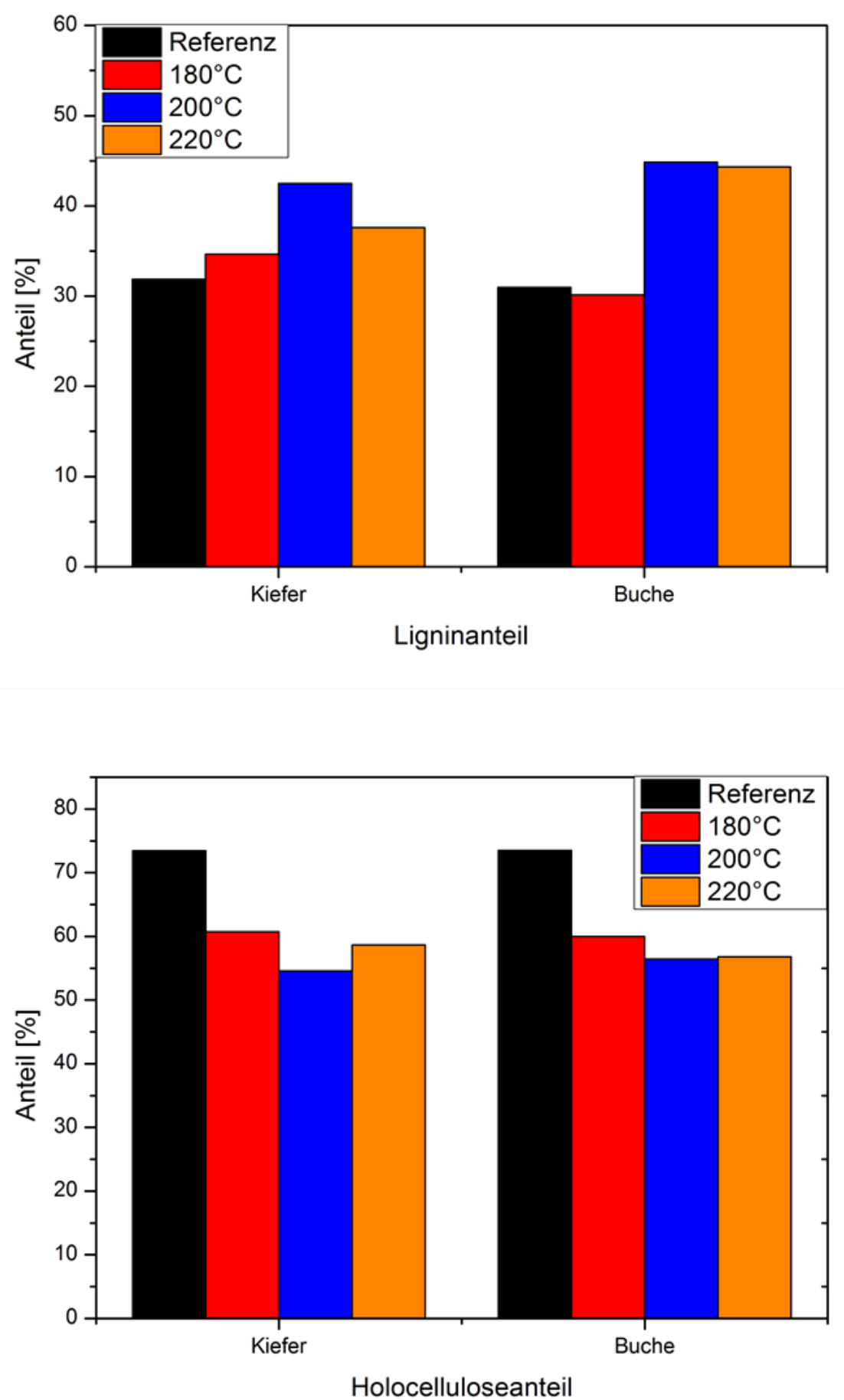

Abb. 2.5: Säulendiagramm vom Lignin-Anteil (oben) und dem Holocellulose-Anteil (unten) in \% für die thermisch modifizierte und extraktfreie Buche bzw. Kiefer mit den Modifikationsparametern: $p_{\bmod } 5 \mathrm{bar}$ und $T_{\bmod } 180^{\circ} \mathrm{C}$ (rot), $200^{\circ} \mathrm{C}$ (blau), $220^{\circ} \mathrm{C}$ (orange) sowie für die Referenz Buche bzw. Kiefer (schwarz). 
im Vergleich zum Verhältnis zu allen anderen Holzkomponenten. Interessanterweise ist der Wert für thermisch modifizierte Kiefer bei $200{ }^{\circ} \mathrm{C}$ erheblich größer als bei $220^{\circ} \mathrm{C}$.

Für die thermisch modifizierte Buche wird dieser Effekt praktisch nicht mehr beobachtet. Die Ergebnisse für die Holocellulose, wobei die Holocellulose eine Masse aus der Cellulose und der Hemicellulose ist, zeigen ein entsprechend umgekehrtes Bild (s. Abb. 2.5 unten). Der Holocellulose-Anteil für die thermisch modifizierte Kiefer bei $200{ }^{\circ} \mathrm{C}$ ist kleiner als bei $220^{\circ} \mathrm{C}$. Auch hier unterscheiden sich die Resultate für die thermisch modifizierte Buche bei $200{ }^{\circ} \mathrm{C}$ sowie bei $220^{\circ} \mathrm{C}$ kaum voneinander.

Daraus ergibt sich, dass die Holzkomponente (bzw. das Lignin) in der Kiefer während der thermischen Modifikation im Temperaturbereich zwischen $200{ }^{\circ} \mathrm{C}$ und $220^{\circ} \mathrm{C}$ sich schneller zersetzen als in der Buche im gleichen Temperaturbereich. Die TGA-Ergebnisse unterstützen diese Hypothese eindeutig.

Detaillierte Änderungen innerhalb der chemischen Struktur des TMT sind auf den FT-IR Spektren (s. die Abb. 2.6) besser zu erkennen. Die Banden sind nach Hao et al. zugeordnet und in der Tabelle 2.2 dargestellt. ${ }^{63}$ Die Normierung für das TMT ist sehr problematisch, weil jede Bande ihre Intensität unter der Hitze theoretisch ändern können. Trotzdem sind die FT-IR Spektren normiert, um den quantitativen Vergleich zu ermöglichen. Dafür sind die stabilsten Banden aus dem aromatischen Bereich für die Buche bei $1455 \mathrm{~cm}^{-1}$ (unten) und für die Kiefer bei $1450 \mathrm{~cm}^{-1}$ (unten) ausgesucht. Infolgedessen ist es potentiell möglich, dass die Spektren kleinere Ungenauigkeiten enthalten.

Eine von den breitesten und intensivsten Banden in dem Wellenzahlbereich zwischen $3590 \mathrm{~cm}^{-1}$ und $3110 \mathrm{~cm}^{-1}$ ist die $\mathrm{OH}-$ Valenzschwingung. Die Fläche der Bande für die Referenzprobe aus der Buche ist die Größte im Vergleich zur TM Buche; für die Buche mit $T_{\bmod } 180{ }^{\circ} \mathrm{C}$ und $200{ }^{\circ} \mathrm{C}$ sind keine große Veränderungen zuerkennen und nur für die Buche mit $T_{\text {mod }} 220^{\circ} \mathrm{C}$ wird die OH-Bande kleiner (s. Abb. 2.6, oben). Für die Referenzprobe aus der Kiefer und der TM Kiefer hat die $\mathrm{OH}-\mathrm{Schwingung}$ eine größere Differenz. Zuerst wird die Intensität durch die Verdampfung des Wassers aus dem Holz und dem Abbau der $\mathrm{OH}-\mathrm{Banden}$ kleiner (s. Abb. 2.6, unten). Danach steigt die Intensität der $\mathrm{OH}-\mathrm{Bande}$ aufgrund der Bildung der $\mathrm{OH}-$ enthaltenden Nebenprodukte $\mathrm{z}$. B. wegen der Zersetzung von $\mathrm{OCH}_{3}-$ Gruppen im Lignin. ${ }^{64}$ 

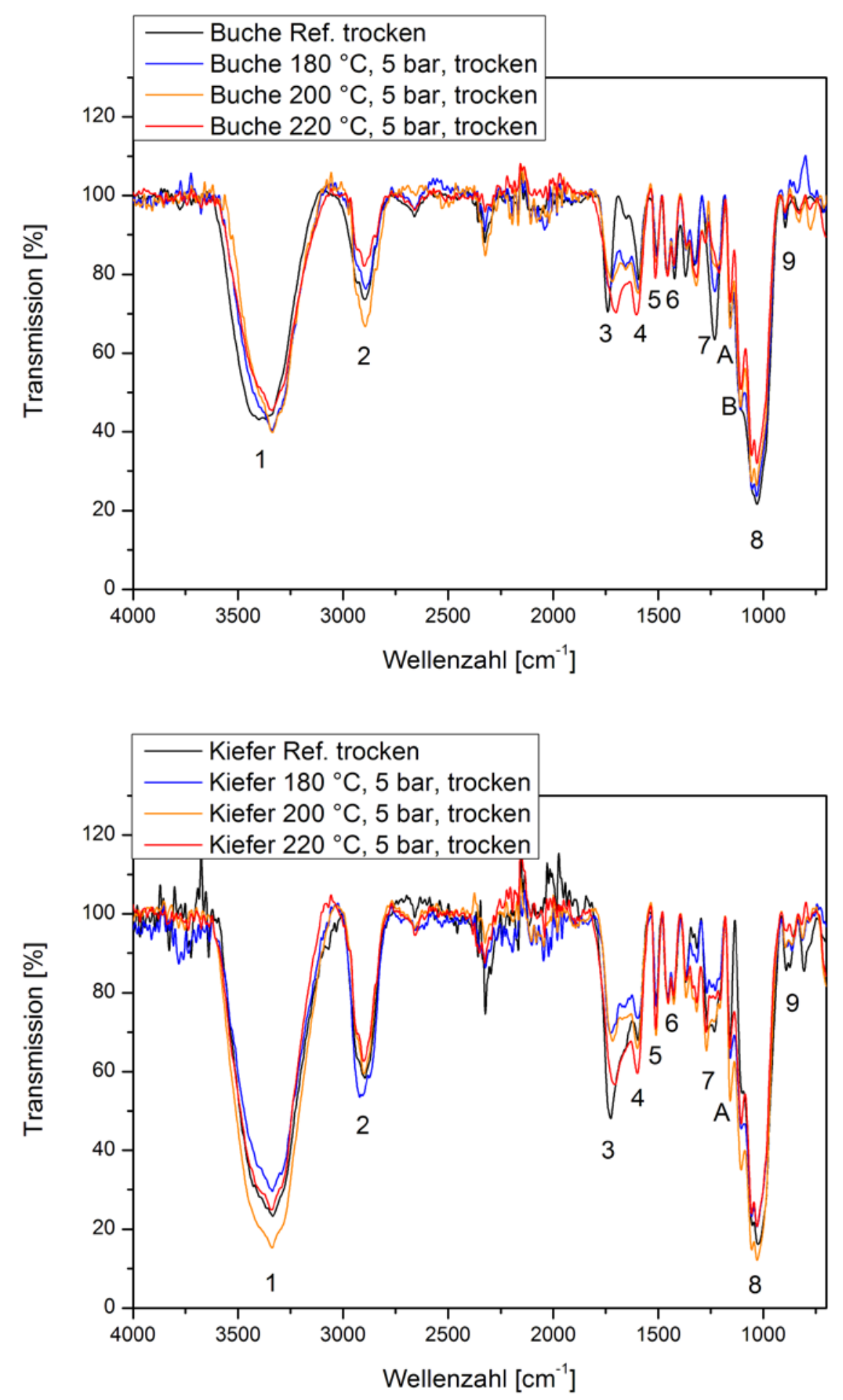

Abb. 2.6: ATR-IR Spektren für Buche (oben) bzw. Kiefer (unten), Die thermisch modifizierten Proben mit dem $p_{\bmod } 5$ bar und $T_{\bmod } 180^{\circ} \mathrm{C}$ (blau), $200{ }^{\circ} \mathrm{C}$ (orange) sowie $220^{\circ} \mathrm{C}$ (rot) sind mit der Referenz Probe (schwarz) verglichen. 
Anschließend wird die Bande wegen des Abbaus der OH-Gruppen unter höherer Temperatur wieder kleiner. Sehr intensive Bande mit der Wellenzahl bei $1740 \mathrm{~cm}^{-1}$ für die Buche und bei $1727 \mathrm{~cm}^{-1}$ für die Kiefer ist die $\mathrm{C}=\mathrm{O}$ Valenzschwingung in Ketonen, Aldehyden und Ester. Die $\mathrm{C}=\mathrm{O}$ Bande nimmt nach der Hitze zuerst stark ab, weil die Hemicellulose abgebaut wird. Danach steigt die $\mathrm{C}=\mathrm{O}$ Bande aufgrund des Abbaus der $\mathrm{OCH}_{3}-$ Gruppen und dadurch die Bildung der neuen $\mathrm{C}=\mathrm{O}$ Bindungen im Lignin wieder.

Tab. 2.2: Hauptbanden in ATR-IR Spektren für das Holz und ihre Zuordnung zu der Funktionalität.63

\begin{tabular}{|c|c|c|c|}
\hline $\begin{array}{l}\text { Wellenzahl } \\
{\left[\mathrm{cm}^{-1}\right]}\end{array}$ & Nr. & Funktionalität & Schwingungen \\
\hline $3590-3100$ & 1 & $\begin{array}{l}\text { OH in Alkohol, } \\
\text { Phenol, Säure }\end{array}$ & Valenzschwingung \\
\hline $2990-2800$ & 2 & $\begin{array}{c}\mathrm{CH}_{3}, \mathrm{CH}_{2}, \mathrm{CH} \\
\text { (Cellulose) }\end{array}$ & Valenzschwingung \\
\hline $1740-1727$ & 3 & $\begin{array}{c}\mathrm{C}=\mathrm{O} \text { in Ester, } \\
\text { Ketone, } \\
\text { Aldehyde }\end{array}$ & $\begin{array}{c}\text { nicht konjugierte } \\
\text { Valenzschwingung }\end{array}$ \\
\hline $1653-1504$ & $4-5$ & $\begin{array}{c}\mathrm{C}=\mathrm{C} \\
\text { (Lignin) }\end{array}$ & $\begin{array}{c}\text { konjugierte } \\
\text { Valenzschwingung }\end{array}$ \\
\hline $1455-1421$ & 6 & $\begin{array}{l}\mathrm{CH} \text { in arom. } \\
\text { Ring }\end{array}$ & Deformationsschwingung \\
\hline $1330-1230$ & 7 & $\begin{array}{l}\mathrm{CO} \text { in } \mathrm{G}-\text { und } \\
\text { S-Lignin } \\
\text { und } \mathrm{OH}\end{array}$ & Valenzschwingung \\
\hline 1140 & A & $\begin{array}{c}\mathrm{CH} \text { in G-Lignin } \\
\text { und } \mathrm{CO}\end{array}$ & \multirow{2}{*}{$\begin{array}{c}\mathrm{CH} \\
\text { Deformationsschwingung in } \\
\text { Lignin und } \\
\text { CO Valenzschwingung }\end{array}$} \\
\hline 1128 & B & $\begin{array}{l}\mathrm{CH} \text { in S-Lignin } \\
\text { und } \mathrm{CO}\end{array}$ & \\
\hline $1046-1023$ & 8 & $\mathrm{C}-\mathrm{O}-\mathrm{C}$ & Deformationsschwingung \\
\hline 840 & 9 & $\begin{array}{l}\mathrm{CH} \text { in } p \text {-Position } \\
\text { in (S-Lignin) }\end{array}$ & Deformationsschwingung \\
\hline
\end{tabular}


Die konjugierten $C=C$ Valenzschwingungen liegen zwischen $1653 \mathrm{~cm}^{-1}$ und $1504 \mathrm{~cm}^{-1}$. CH Deformationsschwingungen in Aromaten befinden sich zwischen $1455 \mathrm{~cm}^{-1}$ und $1421 \mathrm{~cm}^{-1}$. Die Bande mit der größten Absorption gehört zu den C-O-C Deformationsschwingungen und liegt zwischen $1046 \mathrm{~cm}^{-1}$ und $1023 \mathrm{~cm}^{-1}$.

Auch die IR Spektren unterstützen die oben genannte Hypothese- dass die Holzkomponente (bzw. das Lignin) in der Kiefer während der thermischen Modifikation im Temperaturbereich bei $200{ }^{\circ} \mathrm{C}$ sich schneller zersetzt als in der Buche.

Da das Holz ein sehr komplexes Material ist und aus mehreren Komponenten besteht, die sich während der thermischen Modifikation gegenseitig ständig beeinflussen, sind die FT-IR Resultate deutlich schwerer $\mathrm{zu}$ interpretieren. Aus diesem Grund wird das System vereinfacht und das Lignin im Kapitel 3 separat untersucht.

\subsubsection{Massenverlust (ML)}

Die Behandlung vom Holz durch die Hitze führt zu einem Massenverlust (ML). Dies ist von den Einwirkungsmethoden, der Temperatur, dem Druck und der Holzart abhängig. 65,66

Wie in der Abb. $2.7 \mathrm{zu}$ sehen, ist der Massenverlust im thermisch modifizierten Holz direkt proportional zu der Modifikationstemperatur und zum Modifikationsdruck. Im Bereich von den niedrigen Behandlungstemperaturen ist der Massenverlust sehr klein. Hauptsächlich wird dies durch den Verlust von den flüchtigen Stoffen und dem gebundenen Wasser erklärt. ${ }^{67}$ Größere Zersetzung von den makromolekularen Holzkomponenten und der entsprechende Massenverlust liegt in dem Bereich deutlich über $100^{\circ} \mathrm{C}$.

In der Tab. 2.3 sind die ML-Werte für die thermisch modifizierte Buche bzw. die Kiefer in der Abhängigkeit von der Behandlungstemperatur und dem Druck dargestellt. Es ist deutlich zu erkennen, die Buche hat den größeren Massenverlust als die Kiefer. Dieses wird durch den größeren Hemicellulose-Anteil in der Buche erklärt. Wie schon oben gezeigt wurde, wird die Hemicellulose schneller abgebaut als andere Komponenten (s. Abb. 2.1). Mit der Erhöhung des Druckes werden alle Zersetzungsprozesse beschleunigt und der Massenverlust wird immer größer. Nur die Werte in dem Temperaturbereich zwischen $180{ }^{\circ} \mathrm{C}$ und $200{ }^{\circ} \mathrm{C}$ für den Prozess unter dem atmosphärischen Druck haben einen 
anderen Trend. Hier ist ML in der Kiefer etwas größer als ML in der Buche.

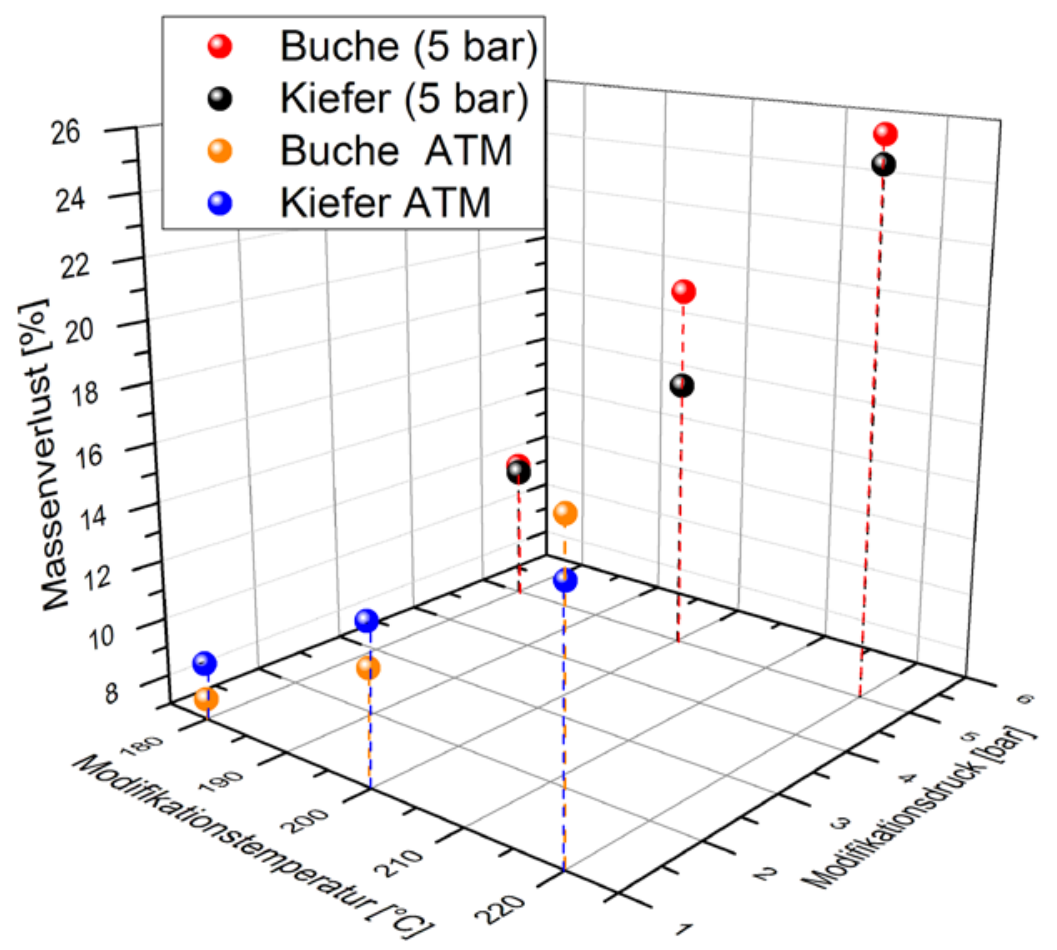

Abb. 2.7: 3D-Auftragung des Massenverlustes in \% gegen die Modifikationstemperatur in ${ }^{\circ} \mathrm{C}$ und gegen den Modifikationsdruck in bar für die Buche (rot) und die Kiefer (schwarz) aus dem geschlossenen Prozess mit dem Druck von 5 bar, und für die Buche (orange) und die Kiefer (blau) aus dem offenem Prozess.

Tab. 2.3: Massenverlust (ML) in \% für die Buche bzw. die Kiefer nach der thermischen Modifikation mit $T_{\bmod } 180^{\circ} \mathrm{C}, 200^{\circ} \mathrm{C}$ und $220^{\circ}$, für einen geschlossenen Prozess mit dem Druck von 5 bar und einen Prozess mit dem Druck von 1 bar.

\begin{tabular}{|c|c|c|c|}
\hline $\boldsymbol{T}_{\text {mod }}\left[{ }^{\circ} \mathbf{C}\right]$ & $p_{\text {mod }}[$ bar $]$ & $\begin{array}{c}\text { ML Buche } \\
{[\%]}\end{array}$ & $\begin{array}{c}\text { ML Kiefer } \\
{[\%]}\end{array}$ \\
\hline 180 & 5 & 12,0 & 11,8 \\
\hline 200 & 5 & 19,8 & 16,5 \\
\hline 220 & 5 & 25,8 & 24,9 \\
\hline 180 & 1 & 7,7 & 8,9 \\
\hline 200 & 1 & 10,9 & 12,4 \\
\hline 220 & 1 & 17,4 & 15,5 \\
\hline
\end{tabular}

Die Ergebnisse des Massenverlustes unterstützen auch die aufgestellte Hypothese - dass die Holzkomponente (bzw. das Lignin) in der Kiefer sich 
während der thermischen Modifikation im Temperaturbereich bei $200{ }^{\circ} \mathrm{C}$ schneller zersetzt als in der Buche.

\subsubsection{Wasserkontaktwinkel (WCA)}

Neben der thermischen Zersetzung und dem Massenverlust ist die Hydrophobizität des TMT auch eine wichtige Eigenschaft, die in dieser Arbeit untersucht wird.

Der Wasserkontaktwinkel (WCA) zeigt die Abhängigkeit der Hydrophobizität des thermisch modifizierten Holzes von den Modifikationsparametern (s. Abb. 2.8). Mit der steigenden Modifikationstemperatur wird die Hydrophobizität des Holzes deutlich erhöht. Die hydrophile Hemicellulose wird durch die Hitze stark abgebaut (s. Abb. 2.1). Dies führt zur niedrigen Adsorption des Wassers von der Oberfläche des Holzes. Die TM Kiefer ist hydrophober als die TM Buche, weil der hydrophile Hemicellulose-Anteil in der Kiefer geringer als in der Buche ist. ${ }^{8}$
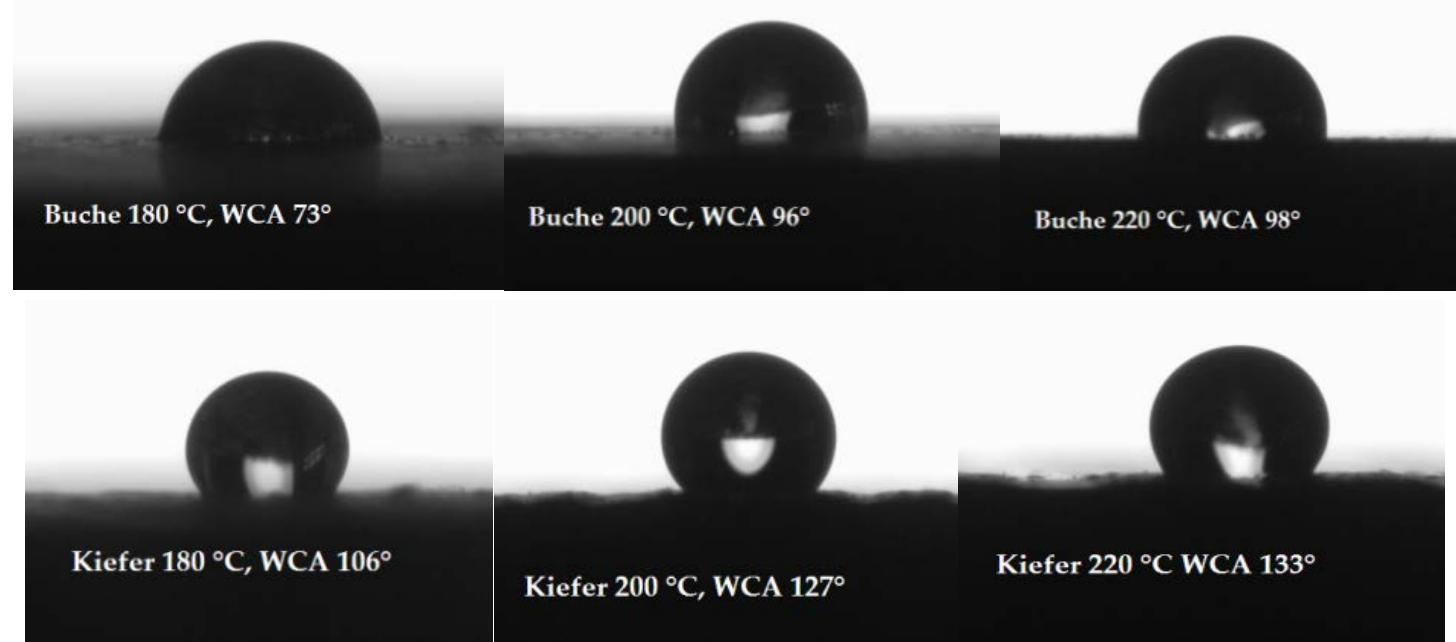

Abb. 2.8: Wasserkontaktwinkelmessungen von der thermisch modifizierten Buche (oben) mit $T_{\text {mod }} 180^{\circ} \mathrm{C}, 200^{\circ} \mathrm{C}, 220^{\circ} \mathrm{C}$ und $p_{\text {mod }} 5$ bar und von der thermisch modifizierten Kiefer (unten) mit $T_{\bmod } 180^{\circ} \mathrm{C}, 200^{\circ} \mathrm{C}, 220^{\circ} \mathrm{C}$ und $p_{\bmod } 5$ bar.

Das nicht modifizierte Holz ist sehr hydrophil und adsorbiert das Wasser sofort. Mit den steigenden Modifikationsgraden des Holzes bleibt das 
Wasser länger an der Oberfläche und wird langsamer adsorbiert. Für die Proben mit $T_{\bmod } 200{ }^{\circ} \mathrm{C}$ und $220^{\circ} \mathrm{C}$ bleibt das Wasser an der Oberfläche länger als 5 Minuten oder wird kaum adsorbiert.

\subsection{Stabile freie Radikale im TMT}

Eine der typischen charakteristischen Eigenschaften vom thermisch modifizierten Holz ist das Vorhandensein der freien Radikalen. Diese Radikale weisen eine sehr hohe Stabilität auf, so dass sie auch noch nach mehreren Monaten nachzuweisen sind (s. Abs. 2.2.2).

Elektronenspinresonanz (ESR) Spektroskopie ist eine häufig angewendete Untersuchungsmethode für die freien Radikalen. In dieser Arbeit ist sie die Hauptmethode und wird deswegen etwas genauer erklärt.

\subsubsection{Auswertung von ESR Spektren}

Ein Standard ESR Spektrum für eine Holzprobe ist auf der Abb. 2.9 dargestellt. In diesem Fall ist es das Spektrum für die TM Buche mit der Modifikationstemperatur $220^{\circ} \mathrm{C}$ und dem Modifikationsdruck 5 bar. Die Messung wird bei Raumtemperatur und in einem X-Bande Bereich mit der Frequenz von 9,42 GHz durchgeführt. Die ESR Messungen in dieser Arbeit zeigen, dass alle ESR Signale für das Holz sehr ähnlich sind. Dieses ist unabhängig von der Holzart und den Modifikationsbedingungen. Es ist immer ein sehr symmetrischer und relativ breiter Peak ohne Feinstruktur. Der Grund dafür ist, dass alle Holzproben als Festproben gemessen werden. Sie sind ziemlich schwer zu lösen oder sind komplett unlöslich. Die Komplexität der Interpretation des Spektrums und dadurch der Charakterisierung des Radikals liegt in der Tatsache, dass alle Signale von den verschiedenen Radikalen zu einem großen Peak summiert werden. Trotzdem liefert diese Methode die wichtige Information über die Konzentration der Radikalen und charakteristische Werte den g-Faktor, 

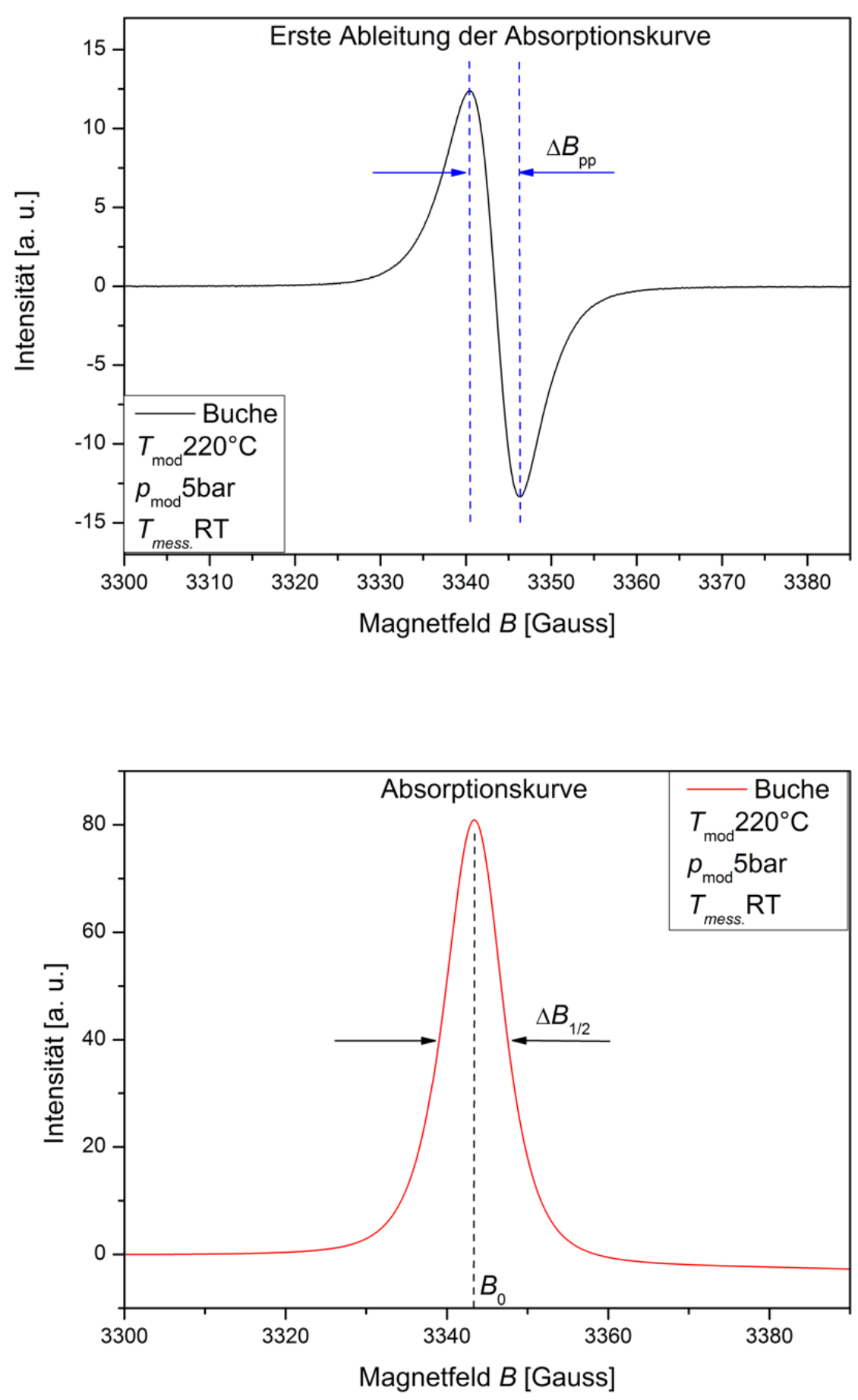

Abb. 2.9: Typisches ESR Spektrum für das Holz als eine Absorptionskurve (unten, rot) und ihre erste Ableitung (oben, schwarz) für die TM-Buche mit der Modifikationstemperatur $220^{\circ} \mathrm{C}$ und dem Modifikationsdruck 5 bar. Die Messung wurde bei Raumtemperatur und mit der Frequenz von 9,42 GHz durchgeführt. 
den Abstand zwischen den positiven und negativen Peak $\Delta B_{\mathrm{pp}}$, die Linienbreite auf halbe Höhe $\Delta B_{1 / 2}$ und die Fläche unter der Absorptionskurve.

Zur Bestimmung des g-Faktors wird die Gleichung 2.1 angewendet,

$$
g=\frac{h v}{\mu_{\mathrm{B}} B_{0}}
$$

wobei $h$ die Planck Konstante, $v$ die Frequenz, $\mu_{\mathrm{B}}$ Bohr Magneton Moment und $B_{0}$ magnetisches Feld am Maximum der Absorptionskurve sind. ${ }^{68}$ Das Signal zentriert sich auf ein Resonanzfeld mit dem $g$-Faktor von ca. 2,002. Dieser Wert entspricht dem $g$-Faktor für die organische freie Radikale. ${ }^{69}$

Das Absorptionssignal wird als erste Ableitung der Absorptionskurve angezeigt (s. Abb. 2.9, oben). Die wichtigste Information liefert die Fläche unter der Absorptionskurve (s. Abb. 2.9, unten). Sie ist proportional zu der Radikalkonzentration in der gemessenen Probe und wird durch ein doppeltes Integral der ersten Ableitung der Absorptionskurve berechnet, ${ }^{70}$

$$
\iint I \propto[R \cdot]
$$

wobei $I$ die Intensität der Absorptionskurve und [ $R$ •] die Konzentration der freien Radikalen in der Probe sind.

\subsubsection{Bildung von stabilen freien Radikalen in TMT}

Altgen et. al. haben in ihrer Arbeit bestimmt, die ESR Signalintensität der Radikalen in TMT korreliert sehr eng mit der Behandlungsintensität. ${ }^{71}$ Deswegen werden in dieser Arbeit die unterschiedlichen Faktoren untersucht, die die Bildung von stabilen freien Radikalen in TMT beeinflussen.

In der Abb. 2.10 ist es deutlich zu erkennen, die Parameter der thermischen Modifikation, wie $T_{\text {mod }}$ oder $p_{\text {mod, }}$ spielen eine große Rolle. 
So z. B. mit der steigenden Modifikationstemperatur bilden sich immer mehr freie Radikale, weil die Anzahl der gebrochenen Bindungen in der molekularen Struktur des Holzes unter der Hitze immer weiter steigt. Signifikante Unterschiede sind in dem Temperaturbereich ab $200^{\circ} \mathrm{C}$ und höher zu sehen. Wie schon in dem Abs. 2.1.1 bestimmt wurde, beginnt Lignin in diesem Temperaturbereich sich stark abzubauen. Das beweist, dass der größte Anteil von den freien stabilen Radikalen im TMT aus dem Lignin vorkommt. Die Stabilität der Radikalen wird durch den delokalisierten Elektronenzustand in der konjugierten Ligninstruktur und durch die begrenzte Beweglichkeit der Substituenten erklärt. ${ }^{72,73}$

Die Anzahl der freien stabilen Radikalen ist direkt proportional zum Modifikationsdruck (s. Abb. 2.10). Der Druck während der thermischen Modifikation beschleunigt die Zersetzungsprozesse, so dass die Intensität der Radikalen steigt.

Interessanterweise liefern die Holzarten auch unterschiedliche Ergebnisse. Wesentlich mehr freie Radikale bilden sich im Laubholz als im Nadelholz (s. Abb. 2.10).

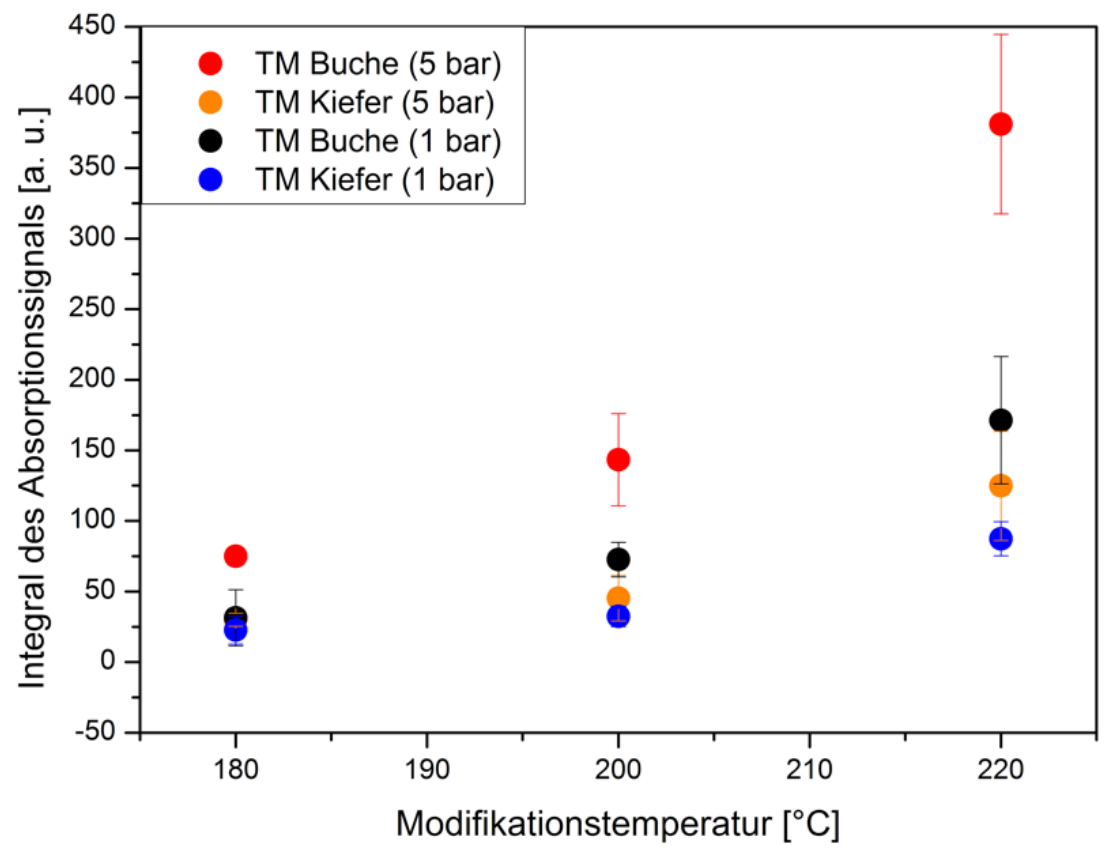

Abb. 2.10: Auftragung des Integrals des Absorptionssignals gegen die Modifikationstemperatur in ${ }^{\circ} \mathrm{C}$ für die Buche (rot) und die Kiefer (orange) aus dem geschlossenem Prozess mit dem Druck 5 bar, und für die Buche (schwarz) und die Kiefer (blau) aus dem offenem Prozess mit dem Druck 1 bar. 
Die größte Differenz liegt im Temperaturbereich bei $220^{\circ} \mathrm{C}$. Gerade in diesem Bereich wird das Lignin stark abgebaut (s. Abs. 2.1). Auch diese Ergebnisse werden durch den unterschiedlichen chemischen Aufbau der Buche und der Kiefer erklärt, genauer gesagt durch die unterschiedliche Ligninstruktur im Laub- bzw. im Nadelholz. Das Lignin aus der Buche enthält deutlich mehr Methoxy-Gruppen als das Lignin aus der Kiefer. ${ }^{74}$ Die Methoxy-Gruppen werden unter der steigenden Hitze bzw. dem Druck schneller abgebrochen und entstehen OH-Gruppen. (s. Kapitel 3).

Auch nicht modifizierte Holzproben (noTM) enthalten stabile freie Radikale (s. Abb. 2.11, blau und orange). Die Konzentration der Radikalen ist ähnlich wie für das TMHolz mit den niedrigen Modifikationstemperaturen unter $180^{\circ} \mathrm{C}$. Das Vorhandensein von freien Radikalen im nicht modifizierten Holz wird auch durch eine Biosynthese des Lignins erklärt. ${ }^{75}$ Durch eine Dehydrierung des Coniferylalkohols während der Polymerisation entsteht ein Dehydroconiferylalkohol-Radikal (s. Schema 2.1). ${ }^{76}$
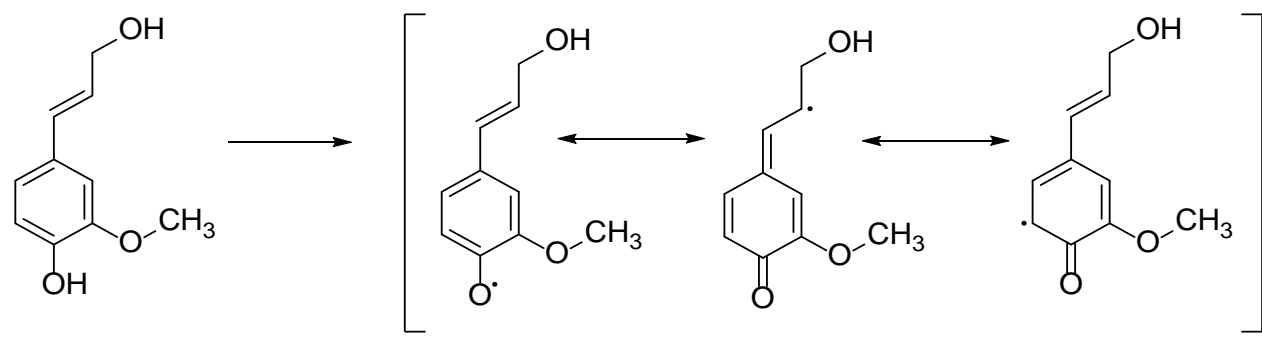

Schema 2.1: Entstehung eines Dehydroconiferylalkohol-Radikals durch eine Dehydrierung des Coniferylalkohols während der Polymerisationsreaktion.

Die Messreihen für die TM Buche und die TM Kiefer mit den unterschiedlichen relativen Luftfeuchtigkeiten ( $\mathrm{RH}$, englisch Relative Humidity) über längere Zeit zeigen sehr interessante Ergebnisse. Direkt nach der thermischen Modifikation $\left(T_{\bmod } 220^{\circ} \mathrm{C}, p_{\bmod } 5\right.$ bar) wurden die Holzproben über die längere Zeit in den Klimaschränken bei der Luftfeuchtigkeit 30\% und 85\% gelagert. Die ESR-Messreihen wurden 5 Wochen lang durchgeführt. Die Masse jeder Probe enthält $1 \mathrm{~g}$. In der Abb. 2.11 sind die Werte der Integralen des Absorptionssignals gegen die Zeit nach der thermischen Modifikation in der Abhängigkeit der relativen Luftfeuchtigkeit mit 30\% und $85 \%$ dargestellt, wobei 0 auf der X-Achse das Ende der Modifikationsprozesse ist. 

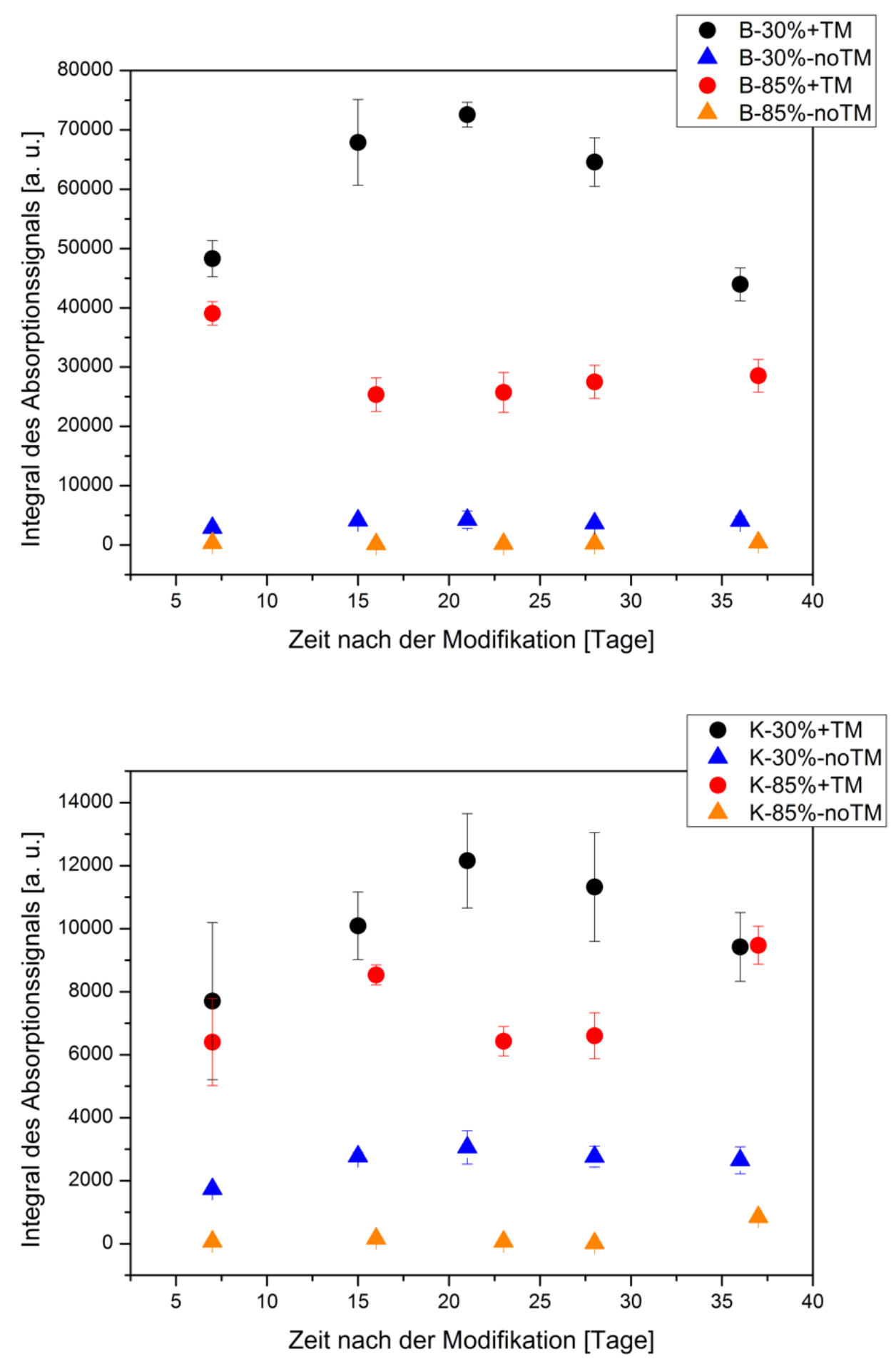

Abb. 2.11: Auftragung des Integrals des Absorptionssignals gegen Zeit nach der thermischen Modifikation in Tagen, wobei 0 Tage das Ende der thermischen Modifikation ist, für TM Buche und nicht TM Buche (oben); und TM Kiefer und nicht TM Kiefer (unten) mit der relative Luftfeuchtigkeit von $30 \%$ und $85 \%$. Parametern der Modifikation: $T_{\bmod }=220{ }^{\circ} \mathrm{C}$, $p_{\text {mod }}=5$ bar. 
Wie erwartet, zeigen die Proben mit der niedrigeren relativen Luftfeuchtigkeit (s. Abb. 2.11, schwarz) hohe Konzentrationen der freien stabilen Radikalen. Das passt sowohl für die Buche als auch für die Kiefer. Es wird dadurch erklärt, dass das Wasser das ESR Signal absorbiert und dadurch die Intensität der freien stabilen Radikalen deutlich geringer wird. ${ }^{77}$

Der spannende Punkt ist, dass die Konzentration der Radikalen in der Probe mit der niedrigen RH 30\% sowohl für die Buche als auch für die Kiefer die ersten drei Wochen steigt (s. Abb. 2.11, schwarz). Für die TM Proben mit der RH 85\% (s. Abb. 2.11, rot) ist dieser Trend nicht mehr erkennbar. Die Konzentration der freien stabilen Radikalen bleibt relativ konstant. Eine mögliche Erklärung ist, dass die Molekularstruktur des Holzes direkt nach der thermischen Modifikation, folglich nach der Zersetzung der Hemicellulose und mehreren Bindungen im Lignin, immer noch unter einer großen Spannung steht. So können einige Bindungen sich noch über bestimmte Zeit weiter brechen. Proben mit dem höheren Wasseranteil zeigen eine bessere Stabilität in der Struktur. Aufgrund der Wechselwirkung des Wassers mit den OH-Gruppen im Holz bilden sich Wasserstoffbrücken. Natürlich sind diese Bildungen deutlich schwächer als die normalen Atombindungen. Die Bindungsenergie zweier Atome über eine Wasserstoffbrücke liegt in der Größenordnung von $0,1 \mathrm{eV} .{ }^{78}$ Trotzdem hat diese Bindungsenergie einen gravierenden Einfluss auf die Stabilität der molekularen Struktur nach der thermischen Modifikation. 


\subsection{Fazit}

In diesem Kapitel wurde die Auswirkung der thermischen Modifikation auf das Holz und die Änderungen innerhalb der makromolekularen Struktur zwischen der TM Buche und der TM Kiefer dargestellt.

Die isolierten Holzbestandteile wie das Lignin, die Cellulose und die Hemicellulose zeigen andere Ergebnisse als das Holz während der TGA Analyse. Dieses wird durch die synergistischen Abbaureaktionen der Komponenten erklärt. Unter der Temperaturerhöhung starten konkurrierende Prozesse im Holz, die sich gegeneinander beeinflussen. Die Zersetzungsprozesse in den isolierten Bestandteilen verlaufen ohne den Einfluss der anderen Komponenten.

Die TGA Analyse hat die interessante Eigenschaft von TMT gezeigt - die thermische Zersetzung des thermisch modifizierten Holzes wird langsamer als des nicht modifizierten Holzes. Dabei wurde festgestellt, dass die thermische Stabilität direkt proportional $\mathrm{zu}$ den Modifikationsstufen ist. Signifikante Unterschiede der thermischen Zersetzung sind zwischen der TM Buche mit der höheren Modifikationstemperatur und der nicht modifizierten Buche. Diese Eigenschaft vom TMT hat ein großes Potential für die Holzindustrie und sollte weiter in einer anderen Arbeit genauer untersucht werden.

Eine weitere wichtige Eigenschaft des TMT ist die erhöhte Hydrophobizität. Dies wird durch den hitzeinduzierten Hemicelluloseabbau erklärt. Hydrophobe Eigenschaften des Materials steigen direkt proportional zur Modifikationsstufe.

Es konnte gezeigt werden, wie sich das Verhältnis der Holzkomponenten im TMT in der Abhängigkeit von den Modifikationsparametern ändert. Mit der steigenden Modifikationstemperatur steigt der Lignin-Anteil sowohl für die Buche als auch für die Kiefer, weil das Lignin deutlich langsamer als die Hemicellulose und die Cellulose abgebaut wird. Unerwartet waren die Ergebnisse für die TM Kiefer mit den Modifikationstemperaturen $200^{\circ} \mathrm{C}$ und $220^{\circ} \mathrm{C}$. Der Lignin-Anteil für die TM Kiefer mit $T_{\bmod } 220^{\circ} \mathrm{C}$ ist niedriger als für die TM Kiefer mit $T_{\text {mod }} 200^{\circ} \mathrm{C}$. Im Gegensatz dazu zeigen die Holocellulose-Anteile für die TM Kiefer ein entsprechend umgekehrtes Bild und somit unterstützen die Ergebnisse für das Lignin. Für die TM Buche wird dieser Effekt praktisch nicht mehr beobachtet. Daraus wurde die Hypothese aufgestellt - das 
Lignin in der Kiefer während der thermischen Modifikation im Temperaturbereich zwischen $200^{\circ} \mathrm{C}$ und $220^{\circ} \mathrm{C}$ zersetzt sich schneller als in der Buche im gleichen Temperaturbereich. Die TGA-Ergebnisse und die Bestimmung des Massenverlustes nach der thermischen Modifikation unterstützen diese Hypothese eindeutig.

Ein weiterer wichtiger Punkt in diesem Kapitel war die Bildung der freien Radikalen im TMT. Es wurde die Abhängigkeit der ESR Signalintensität der Radikalen von den Modifikationsparametern sowie von der relativen Luftfeuchtigkeit und von der Zeit nach der thermischen Modifikation gezeigt. Die ESR Signalintensität der Radikalen im TMT korreliert sehr eng mit der Modifikationstemperatur, weil die Anzahl der gebrochenen Bindungen im TMT unter der Hitze steigt. ${ }^{71}$ Signifikante Unterschiede sind in dem Temperaturbereich ab $200{ }^{\circ} \mathrm{C}$ und höher zu sehen. In diesem Temperaturbereich beginnt sich das Lignin abzubauen. Deswegen wurde impliziert, dass der größte Anteil von freien stabilen Radikalen in TMT aus dem Lignin kommt. Die Anzahl der freien Radikalen ist direkt proportional zum Modifikationsdruck, weil der Druck während der thermischen Modifikation die Zersetzungsprozesse im Holz beschleunigt und die Konzentration der Radikalen automatisch steigt.

Es wurden signifikante Unterschiede in der ESR Analyse zwischen der Buche und der Kiefer festgestellt. Wesentlich mehr freie Radikale bilden sich im Laubholz als im Nadelholz. Die größte Differenz liegt im Temperaturbereich bei $220^{\circ} \mathrm{C}$ aufgrund des hitzeinduzierten Abbruchs der Methoxy-Gruppen im Lignin, weil das Lignin aus der Buche deutlich mehr Methoxy-Gruppen enthält als das Lignin aus der Kiefer. ${ }^{79}$

Es wurde auch gezeigt, dass die freien Radikalen im TMT sehr stabil sind und auch nach mehreren Monaten immer noch nachzuwiesen sind. Die Stabilität der Radikalen wird durch den delokalisierten Elektronenzustand in der konjugierten Ligninstruktur und durch die begrenzte Beweglichkeit der Substituenten erklärt. 80,81

Unerwartete Ergebnisse gab es für die ESR Messreihen von den Proben mit unterschiedlichen RH gegen die Zeit nach der thermischen Modifikation. Die Konzentration der Radikalen in den TM Proben mit der niedrigen RH 30\% steigt in den ersten drei Wochen. Für die TM Proben mit der RH 85\% ist dieser Trend nicht mehr erkennbar. Eine mögliche Erklärung ist, dass die Molekularstruktur des Holzes direkt nach der thermischen Modifikation immer noch unter einer großen Spannung steht. 
So können sich einige Bindungen noch über eine bestimmte Zeit weiter brechen. Proben mit dem höheren Wasseranteil zeigen eine höhere Stabilität in der Struktur. Wasser und die OH-Gruppen im Holz wechselwirken miteinander und bilden die Wasserstoffbrücken. Diese Bildungen sind sehr schwach. Trotzdem hat diese Bindungsenergie einen gravierenden Einfluss auf die Stabilität der molekularen Struktur nach der thermischen Modifikation.

Die gesamte Analyse in diesem Kapitel hat die Komplexität des Holzes gezeigt. Da die hitzeinduzierten Abbaureaktionen sich stark gegeneinander beeinflussen, sind die Zersetzungsmechanismen sehr schwer $\mathrm{zu}$ interpretieren. Infolgedessen wird das Lignin als einzelne Komponente untersucht und die Ergebnisse sind im Kapitel 3 vorgestellt. 


\section{Kapitel 3 Hitzeinduzierte Strukturänderungen im Lignin}

In diesem Kapitel werden die Veränderungen in der molekularen Ligninstruktur gezeigt, die durch Hitze entstehen. Das Ziel ist die Komplexität des Holzes durch die Untersuchung nur einer Holzkomponente zu vereinfachen, um die Zersetzungsmechanismen und die Radikalbildung im TMT leichter zu interpretieren. Da die freie stabile radikale meistens im Lignin vorkommen (s. Kapitel 2), wird vor allem das Lignin charakterisiert.

\subsection{Untersuchte Lignin-Arten}

In dieser Arbeit werden drei unterschiedlich gewonnene Lignin-Arten (technisches, extrahiertes aus Thermoholz und synthetisiertes Lignin) thermisch behandelt und charakterisiert. Technische und extrahierte Lignine werden aus dem Nadelholz (Kiefer) und aus dem Laubholz (Buche) gewonnen und miteinander charakteristisch verglichen.

Die erste Art ist das industriell gewonnene technische Lignin. Dazu gehört das aus der Buche hergestellte Organosolv Lignin und das aus dem Nadelholz (meistens aus der Kiefer) hergestellte Kraft-Lignin Indulin. Das Kraft Lignin wurde freundlicherweise von Prof. Dr. C. Mai und Dr. Marco Fleckenstein von der Fakultät für Forstwissenschaften und Waldökologie zur Verfügung gestellt. Das Organosolv Lignin wurde von Herrn Unkelbach vom Fraunhofer-Zentrum für Chemisch-Biotechnologische Prozesse CBP aus Leuna präsentiert. Die Herstellungsprozesse sind in dem Kapitel 1 beschrieben. Beide Lignin-Sorten werden im Ofen in dem Temperaturbereich zwischen $150{ }^{\circ} \mathrm{C}$ und $200^{\circ} \mathrm{C}$ thermisch behandelt, analysiert und mit dem nicht behandelten technischen Lignin (weiter als Referenz bezeichnet) verglichen.

Die zweite Art des Lignins ist das extrahierte Lignin aus Thermoholz nach dem Klason-Verfahren. Für die Extraktion wurden thermisch modifizierte Buche bzw. Kiefer verwendet. Die Modifikationsprozesse sind im Kapitel 6 beschrieben. Die Modifikationsparametern sind folgende: $T$ mod. $180{ }^{\circ} \mathrm{C}$, $200{ }^{\circ} \mathrm{C}$ und $220^{\circ} \mathrm{C}$ und $p_{\text {mod }} 5$ bar. Alle Prüfkörper werden zuerst bei der 
niedrigsten Stufe mit Partikelgrößen von 0,2 $\mathrm{mm}$ bis $0,63 \mathrm{~mm}$ pulverisiert und dann im Ofen bei $103{ }^{\circ} \mathrm{C}$ über mehrere Tage getrocknet. Im nächsten Schritt werden die Holzproben von den Extraktstoffen befreit (s. Kapitel 6). Aus dem extraktfreien Holz wird Lignin nach Sluiter et al. Methode gewonnen. ${ }^{82}$ Dafür werden jeweils $300 \mathrm{mg}$ des thermisch modifizierten Holzpulver aus jedem Modifikationsprozess mit $3 \mathrm{~mL}$ 72-\%iger Schwefelsäure bei $30^{\circ} \mathrm{C}$ verrührt, danach mit demineralisiertem Wasser auf $4 \%$ verdünnt und im Autoklav bei $121^{\circ} \mathrm{C}$ für zwei Stunden platziert. Abfiltrierte und mit dem Wasser gewaschene Proben werden im Ofen darrgetrocknet und gravimetrisch bestimmt. Anschließend werden diese Proben charakterisiert und mit dem extrahierten Lignin aus dem nicht modifizierten Holz (weiter als Referenz bezeichnet) verglichen.

Als letzte und dritte Art des Lignins ist das synthetisierte aus Coniferylalkohol Lignin Modell. Die Synthese des Lignin Modells aus Coniferylalkohol erfolgt nach Zutropfverfahren von Tanahashi und Higuchi. ${ }^{83}$ Dazu wird eine Phosphatpufferlösung mit pH-Wert von 6,1 aus den Stammlösungen Kaliumdihydrogenphosphat und di-Natriumhydrogenphosphat angesetzt. Als nächstes werden drei Lösungen vorbereitet. (I) $1 \mathrm{~g}$ Coniferylalkohol wird in $33,33 \mathrm{~mL}$ Aceton gelöst und mit 333,33 mL Phosphatpuffer verdünnt. (II) 1,67 mL $\mathrm{H}_{2} \mathrm{O}_{2}$-Lösung (30\%) werden mit 333,33 mL Pufferlösung verdünnt. (III) 16,67 mg Enzym horseradish, Peroxidase werden in 1 L Puffer gelöst. Lösungen (I) und (II) werden nach dem Zutropfverfahren in der Lösung (III) langsam dazu getropft und bei Raumtemperatur 36 Stunden gerührt. Zum Schluss wird das Produkt abfiltriert, mit dem destillierten Wasser gewaschen und getrocknet. Anschließend wird das synthetisierte Lignin Modell im Ofen bei $150{ }^{\circ} \mathrm{C}$ thermisch behandelt und mit der Referenz verglichen.

Nach der thermischen Behandlung werden alle drei Arten des Lignins mithilfe von unterschiedlichen Analysemethoden charakterisiert. Die Ergebnisse den Analysen sind unten dargestellt.

\section{$3.2 \quad$ FT-IR-Spektroskopie}

Aufgrund der pulverartigen Struktur der Proben können die FT-IR-Messungen für alle drei Lignin-Arten mithilfe von den 
KBr-Presslingen gemacht werden. Die Vorteile dieser Analyse sind die hohe Qualität, die bessere Auflösung der IR-Spektren und der geringere Substanzverbrauch. Für diese Methode wird FT-IR-Apparatur in der Fakultät Naturwissenschaften und Technik der HAWK Hochschule in der Abteilung der Prof. Dr. Gisela Ohms benutzt. Diese Spektren werden im Bereich von $400 \mathrm{~cm}^{-1}$ bis $4000 \mathrm{~cm}^{-1}$ und bei einer Auflösung von $8 \mathrm{~cm}^{-1}$ aufgenommen. Jede Probe wird 10 Mal gescannt.

\subsubsection{IR-Spektroskopie für das technische Lignin}

Die Abb. 3.1 zeigt Unterschiede im molekularen Niveau zwischen den zwei Lignin-Sorten: Organosolv (schwarz), was aus der Buche gewonnen wird; und Indulin (rot), was meistens aus der Kiefer hergestellt wird. Die beiden IR-Spektren werden auf die Bande $1512 \mathrm{~cm}^{-1}$ [7] normiert. Deswegen können sie nicht nur qualitativ, sondern auch quantitativ miteinander verglichen werden.

Die Unterschiede in den Spektren kommen einerseits wegen den unterschiedlichen Strukturen des Lignins im Nadel- bzw. im Laubholz vor; anderseits aufgrund der verschiedenen Herstellungsverfahren (s. Kapitel 1). Die Banden sind nach Faix ${ }^{84}$ zugeordnet und in der Tabelle 3.1 dargestellt. Diese Werte sind gültig für alle FT-IR Spektren, die in diesem Kapitel noch weiter vorkommen.

Das Lignin aus dem Laubholz besteht überwiegend aus G- und S-LigninBausteine, wohingegen das Lignin aus dem Nadelholz um $90 \%$ aus G-Lignin-Baustein besteht. ${ }^{8}$ Diese Unterschiede sind in der Abb. 3.1 deutlich zu sehen. So sind die Banden [2], [3] und [14] für Organosolv intensiver als für Indulin, weil Organosolv mehr Methoxy-Gruppen enthält als Indulin. Die Bande [11], die CH-Schwingungen in S-Lignin beschreiben, ist abwesend für Indulin. Besonders überraschend ist die Bande [12], die COC-Schwingungen in Aryl entsprechen. Diese Bande fehlt praktisch in Organosolv. Die Ursache dafür ist, dass die vorhandenen $\beta$-O-4-Bindungen während der Herstellung des Organosolvs aufgebrochen werden. ${ }^{85}$ 

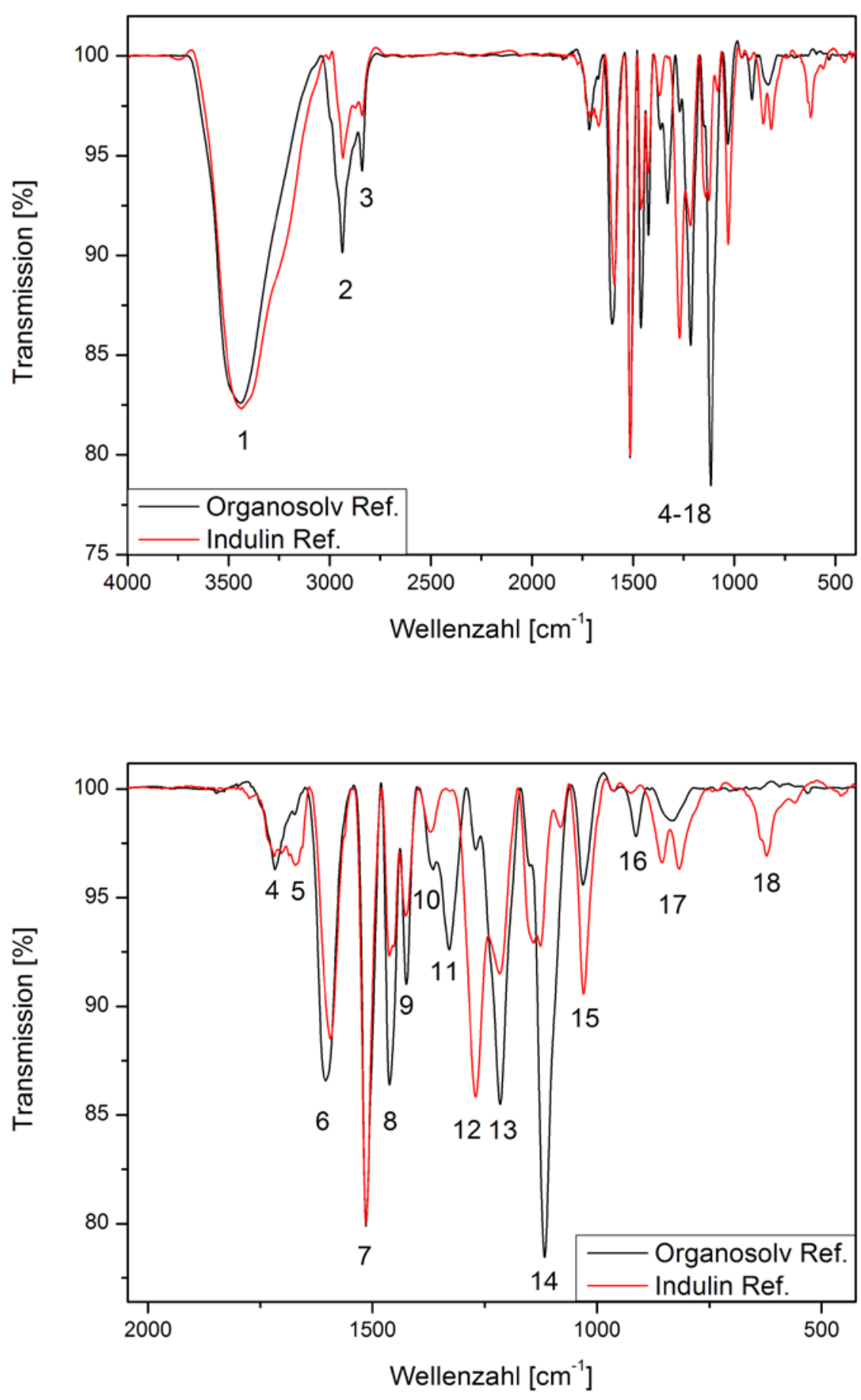

Abb. 3.1: IR Spektrum für die zwei Ligninsorten: Organosolv (schwarz) und Indulin (rot). 
Tab. 3.1: Hauptbanden in FT-IR Spektren für das Lignin und ihre Zuordnung zu der Funktionalität.

\begin{tabular}{|c|c|c|c|}
\hline $\begin{array}{l}\text { Wellenzahl } \\
{\left[\mathrm{cm}^{-1}\right]}\end{array}$ & Nr. & Funktionalität & Schwingungen \\
\hline $3590-3100$ & 1 & $\begin{array}{l}\text { OH in Alkohol, } \\
\text { Phenol, Säure }\end{array}$ & Valenzschwingung \\
\hline $2939 / 2934$ & $2-3$ & $\begin{array}{c}\mathrm{CH}_{3}, \mathrm{CH}_{2}, \mathrm{CH}- \\
\mathrm{sp}^{2} / \mathrm{sp}^{3}\end{array}$ & Valenzschwingung \\
\hline $1711-1670$ & $4-5$ & $\begin{array}{c}\mathrm{C}=\mathrm{O} \text { in Ester, } \\
\text { Ketone, } \\
\text { Aldehyde }\end{array}$ & $\begin{array}{c}\text { nicht konjugierte } \\
\text { Valenzschwingung }\end{array}$ \\
\hline $1603-1543$ & 6-7 & $\begin{array}{c}\mathrm{C}=\mathrm{C} \\
\text { in arom. Ring }\end{array}$ & $\begin{array}{c}\text { konjugierte } \\
\text { Valenzschwingung }\end{array}$ \\
\hline $1453-1427$ & $8-10$ & $\begin{array}{l}\mathrm{CH} \text { in arom. } \\
\text { Ring }\end{array}$ & Deformationsschwingung \\
\hline 1377 & 11 & $\mathrm{CH}$ in S-Lignin & Deformationsschwingung \\
\hline 1272 & 12 & COC in Aryl & Valenzschwingung \\
\hline 1214 & 13 & $\mathrm{OH}$ in. Alkohol & Valenzschwingung \\
\hline 1128 & 14 & $\begin{array}{l}\mathrm{CH} \text { in S-Lignin } \\
\text { und } \\
\mathrm{CO} \text { in Ether }\end{array}$ & $\begin{array}{c}\mathrm{CH} \\
\text { Deformationsschwingung } \\
\text { in S-Lignin und } \\
\text { CO Valenzschwingung }\end{array}$ \\
\hline 1024 & 15 & $\mathrm{CH}$ in G-Lignin & Deformationsschwingung \\
\hline $920-808$ & $16-17$ & $\begin{array}{l}\mathrm{CH} \text { in arom. } \\
\text { Ring }\end{array}$ & Deformationsschwingung \\
\hline
\end{tabular}

In der Abb. 3.2 sind die IR Spektren für Ogranosolv und Indulin dargestellt. Beide Lignin-Sorten werden im Ofen bei $150{ }^{\circ} \mathrm{C}$ (blau) und $200^{\circ} \mathrm{C}$ (rot) 3 Stunden thermisch behandelt und mit den Referenz Proben (schwarz) verglichen. Um die Spektren quantitativ zu vergleichen, werden sie auch auf die Bande [7] bei $1512 \mathrm{~cm}^{-1}$ normiert. Indulin zeigt nach der thermischen Behandlung signifikante Veränderungen in der Struktur und Organosolv ist relativ unverändert. 

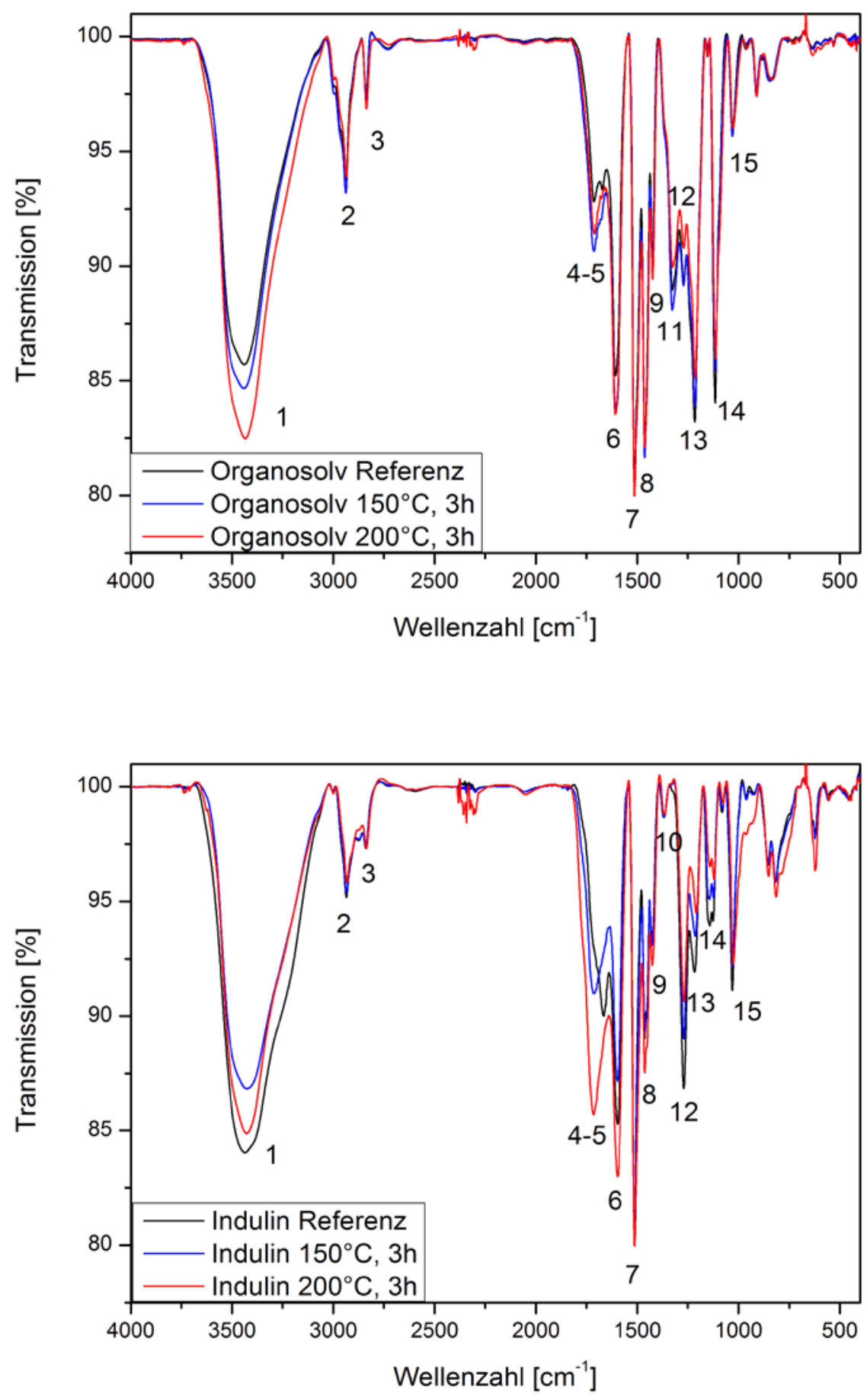

Abb. 3.2: IR Spektren für Organosolv (oben) und Indulin (unten). Das Lignin ist im Ofen bei $150{ }^{\circ} \mathrm{C}$ und $200{ }^{\circ} \mathrm{C} 3$ Stunden thermisch behandelt. Beide Spektren sind auf die Bande [7] normiert. 
Diese Bemerkung unterstützt die Hypothese aus dem Kapitel 2 - das Lignin aus Nadelholz wird schneller abgebaut als aus Laubholz bei der Temperatur $200^{\circ} \mathrm{C}$.

Der aromatische Bereich ist für beiden Lignin-Arten relativ stabil. Dafür nimmt die Bande [14] ab. Diese Bande charakterisiert die Spaltung den COBindungen in Ether. Durch die thermische Oxidation der Alkohole entstehen die Carbonylgruppen. Deswegen wird die starke Zunahme der Bande [4] beobachtet. Dies ist in Indulin deutlicher zu erkennen, weil das Lignin aus der Kiefer in diesem Temperaturbereich schneller abgebaut wird als aus der Buche. Die Bande [12] hat eine deutliche Abnahme mit der steigenden Temperatur und bestätigt den Abbau den $\beta-\mathrm{O}-4-\mathrm{Bindungen} \mathrm{in}$ Indulin. Wie schon erwähnt wurde, sind die $\beta-\mathrm{O}-4$-Bindungen in Organosolv zum größten Teil schon während der Herstellung gebrochen. Deswegen ist die Intensität dieser Bande für Organosolv sehr gering. Mittels der Bande [13] ist deutlich zu erkennen, dass die OH-Bindungen von dem aromatischen Ring und von den Alkoholen in beiden LigninArten durch die Hitze abgebaut werden. Das erklärt die unerwartete Zunahme der $\mathrm{OH}$ Bande [1] für Organosolv bzw. zuerst die Abnahme bei $150^{\circ} \mathrm{C}$ und danach wieder die Zunahme bei $200^{\circ} \mathrm{C}$ der $\mathrm{OH}$-Bande [1] für Indulin. Die Zunahme der Bande [1] entsteht durch die Bildung der neuen $\mathrm{OH}-\mathrm{Gruppen}$, die als Zwischenstufe bei der Spaltung der $\beta$-O-4-Bildungen und der $\mathrm{OCH}_{3}-\mathrm{Gruppen}$ gebildet werden. ${ }^{86}$

\subsubsection{IR-Spektroskopie für das extrahierte Lignin}

Die Abb. 3.3 zeigt die IR Spektren für das extrahierte aus der TM Buche (oben) bzw. aus der TM Kiefer (unten) Lignin. Die Modifikationstemperaturen für das TMT sind $180{ }^{\circ} \mathrm{C}$ (blau), $200^{\circ} \mathrm{C}$ (orange) und $220^{\circ} \mathrm{C}$ (rot). Die Werte aus der Tabelle 3.1 werden auch für diese IR-Auswertungen angewendet.

In dieser Abbildung ist deutlich zu erkennen, dass die Struktur des Lignins stark verändert wurde. Schon für die Referenz Probe (schwarz) ist das der Fall. Dies wird durch die Behandlung mit der konzentrierten Säure erklärt. Die Proben, die aus der Buche extrahiert wurden, zeigen keine signifikante Veränderung in der Struktur. Nur die OH-Bande ändert ihre Intensität und sinkt, weil die hydrophilen Hemicellulosen durch die Hitze zersetzt werden und das TM Holz wenig Wasser speichert. 

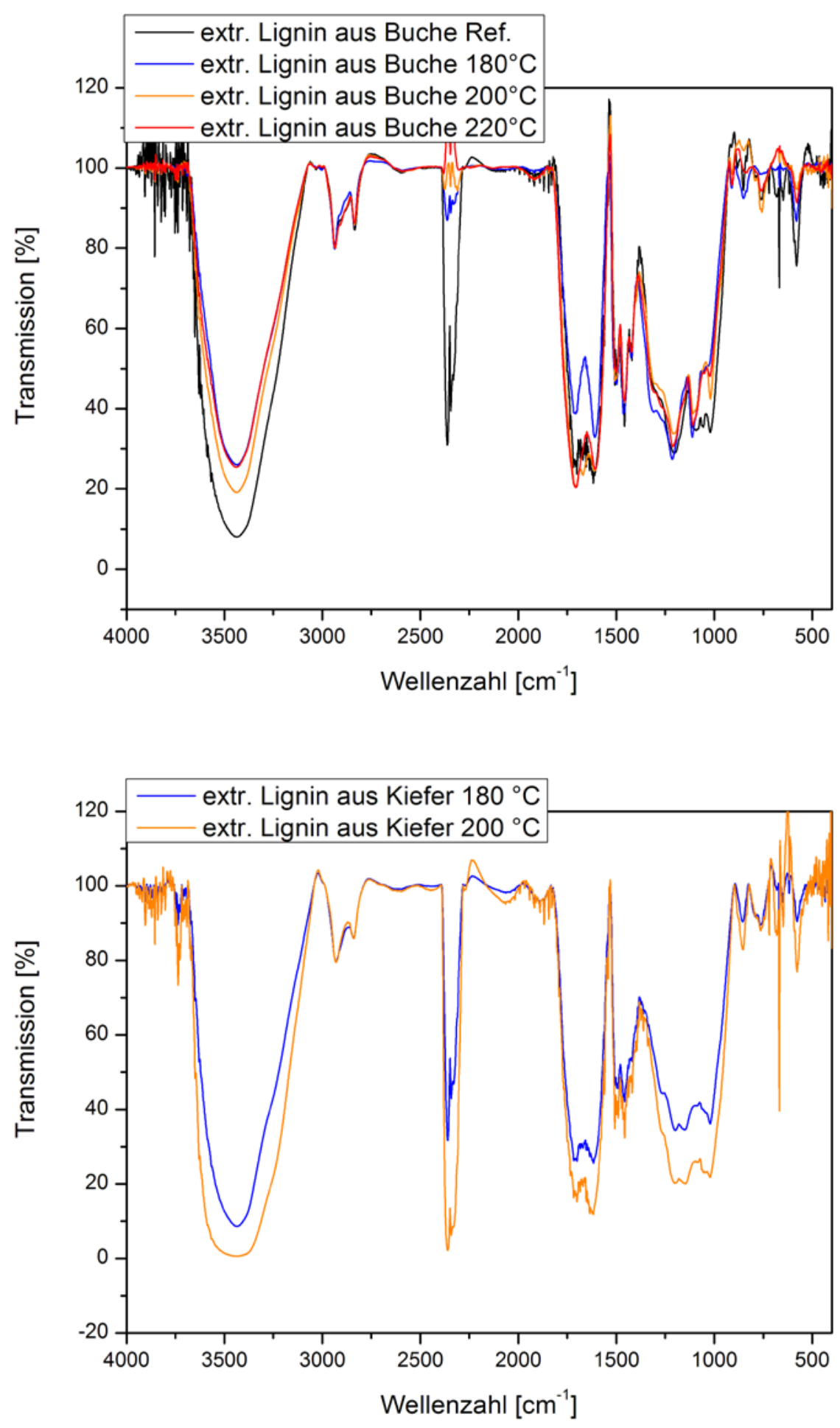

Abb. 3.3: IR Spektren für das extrahierte aus der TM Buche (oben) bzw. aus der TM Kiefer (unten) Lignin, wobei die Modifikationstemperaturen $180^{\circ} \mathrm{C}$ (blau), $200{ }^{\circ} \mathrm{C}$ (orange), $220^{\circ} \mathrm{C}$ (rot) und die Referenz Probe (schwarz) sind. 
Die Proben, die aus der TM Kiefer extrahiert wurden, haben die zunehmende OH-Bande. Da die Zersetzungsprozesse in der Kiefer bei $200^{\circ} \mathrm{C}$ schneller verlaufen als in der Buche, bilden sich mehr neue $\mathrm{OH}-\mathrm{Gruppen}$, die als Zwischenprodukt bei der Spaltung $\beta$-O-4-Bindungen und der $\mathrm{OCH}_{3}-$ Gruppen entstehen. ${ }^{86}$

Bei den zwei Proben, die aus Kiefer extrahiert wurden, gestalten sich die IR Auswertungen schwierig (s. Anhang). Es kann mit diesen Auswertungen keine Aussage getroffen werden, deswegen werden sie in der Abb.3.3 nicht dargestellt.

\subsubsection{IR-Spektroskopie für das synthetisierte Lignin Modell aus dem Coniferylalkohol}

In der Abb. 3.4 sind die FT-IR Spektren des synthetisierten aus dem Coniferylalkohol Lignin Modells (schwarz), des Organosolv Lignins (blau) und des Indulin Lignins (rot) miteinander verglichen.

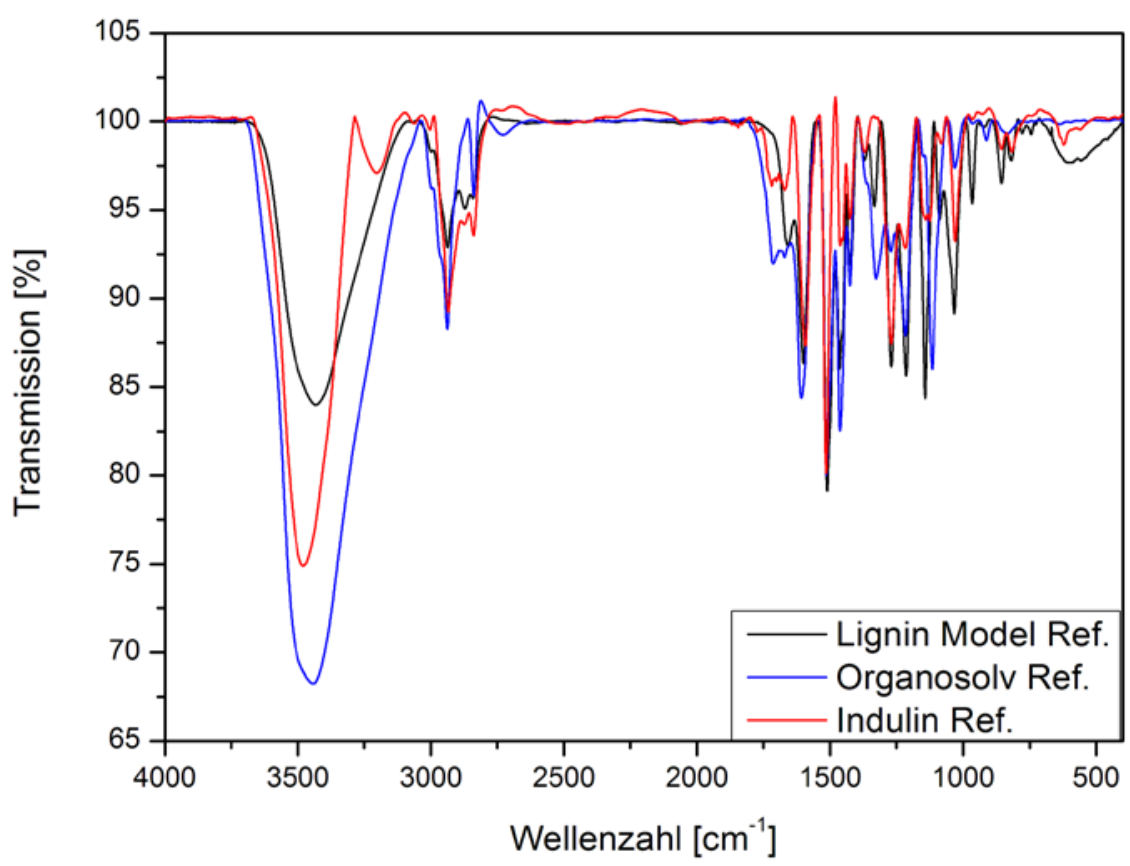

Abb. 3.4: IR Spektren vom synthetisierten aus der Coniferylalkohol Lignin Modell (schwarz), vom Organosolv Lignin (blau) und vom Indulin Lignin (rot). 
Diese Spektren sind auf die Bande bei $1543 \mathrm{~cm}^{-1}$ normiert. Die Struktur des Lignin Modells hat große Ähnlichkeit mit der Struktur des Indulin Lignins. Dies war zu erwarten, weil der Coniferylalkohol ein Bestandteil vom Nadelholz-Lignin ist $^{87}$ und Indulin meistens aus der Kiefer hergestellt wird.

In der Abb. 3.5 sind die FT-IR Spektren dargestellt, diese zeigen die Veränderung durch die thermische Behandlung im Ofen bei $150^{\circ} \mathrm{C}$ (rot) und $200{ }^{\circ} \mathrm{C}$ (blau) in der Struktur des Lignin Modells. Dieses Mal sind die FT-IR Spektren auf die Bande [2] normiert. Da es keine Literaturquelle für die Normierung des thermisch behandelten Lignin Modells gibt, wurden unterschiedliche Banden dafür ausprobiert. Die Banden [2] und [7] zeigen ähnliche Ergebnisse. Die Unterschiede in der Struktur des Lignins werden deutlicher, wenn die Normierung auf die Bande [2] folgt. Infolgedessen werden die FT-IR Spektren für das Lignin Modell auf diese Bande normiert.

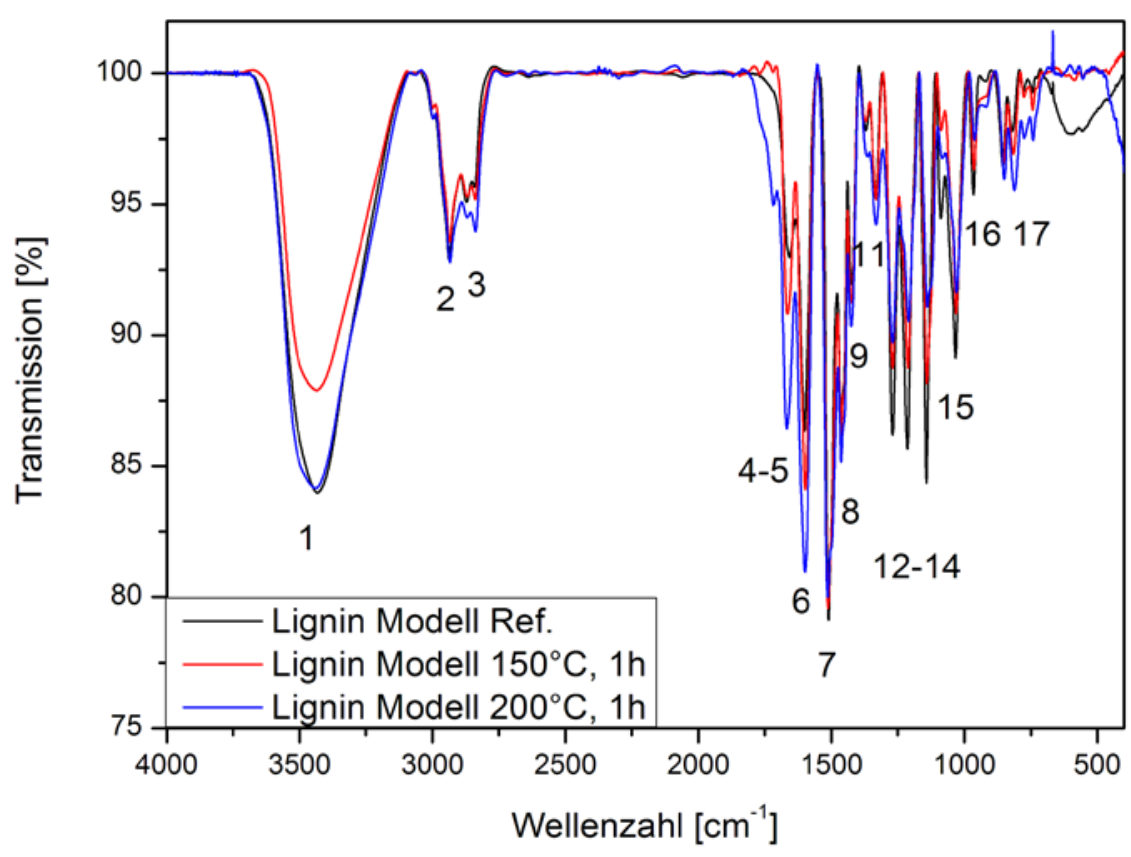

Abb. 3.5: IR Spektren für das synthetisierte aus der Coniferylalkohol Lignin Modell. Das Lignin Modell wurde bei $150{ }^{\circ} \mathrm{C}$ (rot) und $200{ }^{\circ} \mathrm{C}$ (blau) behandelt und mit der Referenz Probe (schwarz) verglichen.

Die Strukturänderungen im Lignin Modell verlaufen ähnlich wie im 
technischen Lignin und sind zu vergleichen mit den Änderungen im TMT. Die Bande [14], die die Spaltung der CO-Bindungen in Ether charakterisiert, nimmt stark ab. Durch die thermische Oxidation der Alkohole lassen sich die Carbonyl-Gruppen herstellen. Deswegen steigt die Intensität der Bande [4] so stark. Die Banden [12-15] haben eine deutliche Abnahme mit der steigenden Temperatur. Das entspricht die Zersetzung der $\beta-\mathrm{O}-4-\mathrm{Bindungen}$, den $\mathrm{OH}$-Bindungen des aromatischen Ringes und der Alkohole. Die Zunahme der Bande [1] bei $200{ }^{\circ} \mathrm{C}$ entsteht durch die Bildung der neuen OH-Gruppen, die als Zwischenstufe beim Abbau der $\mathrm{OCH}_{3}-$ Gruppen gebildet werden.

Der Unterschied zwischen dem Lignin und dem TMT liegt meist in der $\mathrm{C}=\mathrm{O}$ Bande [4]. Im TMT wird die Intensität der $\mathrm{C}=\mathrm{O}$ Bande zuerst sinken, weil die Hemicellulose unter der Hitze abgebaut wird. Erst dann steigt die Intensität aufgrund der Bildung der neuen $\mathrm{C}=\mathrm{O}$ Gruppen nach dem Abbau den $\mathrm{OCH}_{3}-G r u p p e n$. Da das Lignin pur analysiert wird, steigt die Intensität der $\mathrm{C}=\mathrm{O}$ Bande mit der steigenden Temperatur permanent.

\subsection{NMR-Spektroskopie}

Mit Hilfe der NMR Analyse wurden das technische Lignin und das synthetisierte Lignin Modell charakterisiert. Alle Proben wurden mit der Konzentration von $20 \mathrm{mg}$ in $2 \mathrm{~mL}$ DMSO-d 6 vorbereitet. Leider sind nicht alle untersuchten Proben in diesem Lösungsmittel gut löslich. Aus diesem Grund konnten einige Daten nicht ermitteln werden. Trotzdem reichen die ermittelten Daten aus, um ein ausführliches Bild über die Zersetzungsprozesse im Lignin zu bekommen.

\subsubsection{Unterschiede in den NMR-Spektren zwischen dem Ogranosolv und dem Indulin}

In der Abb. 3.6 sind die ${ }^{1} \mathrm{H}-\mathrm{NMR}$ Spektren von Indulin und Organosolv Ligninen dargestellt. Die chemische Verschiebung $\delta$ und die entsprechende Ordnung zur Funktionalität im Lignin sind nach $\mathrm{Li}^{88}$ und Lundquist ${ }^{89,90}$ zugeordnet und in der Tab. 3.2 eingegeben. Bei dem direkten Vergleich der Referenz Proben des Organosolvs und des Indulins lassen sich die weiteren Unterschiede in den Strukturen erkennen. 


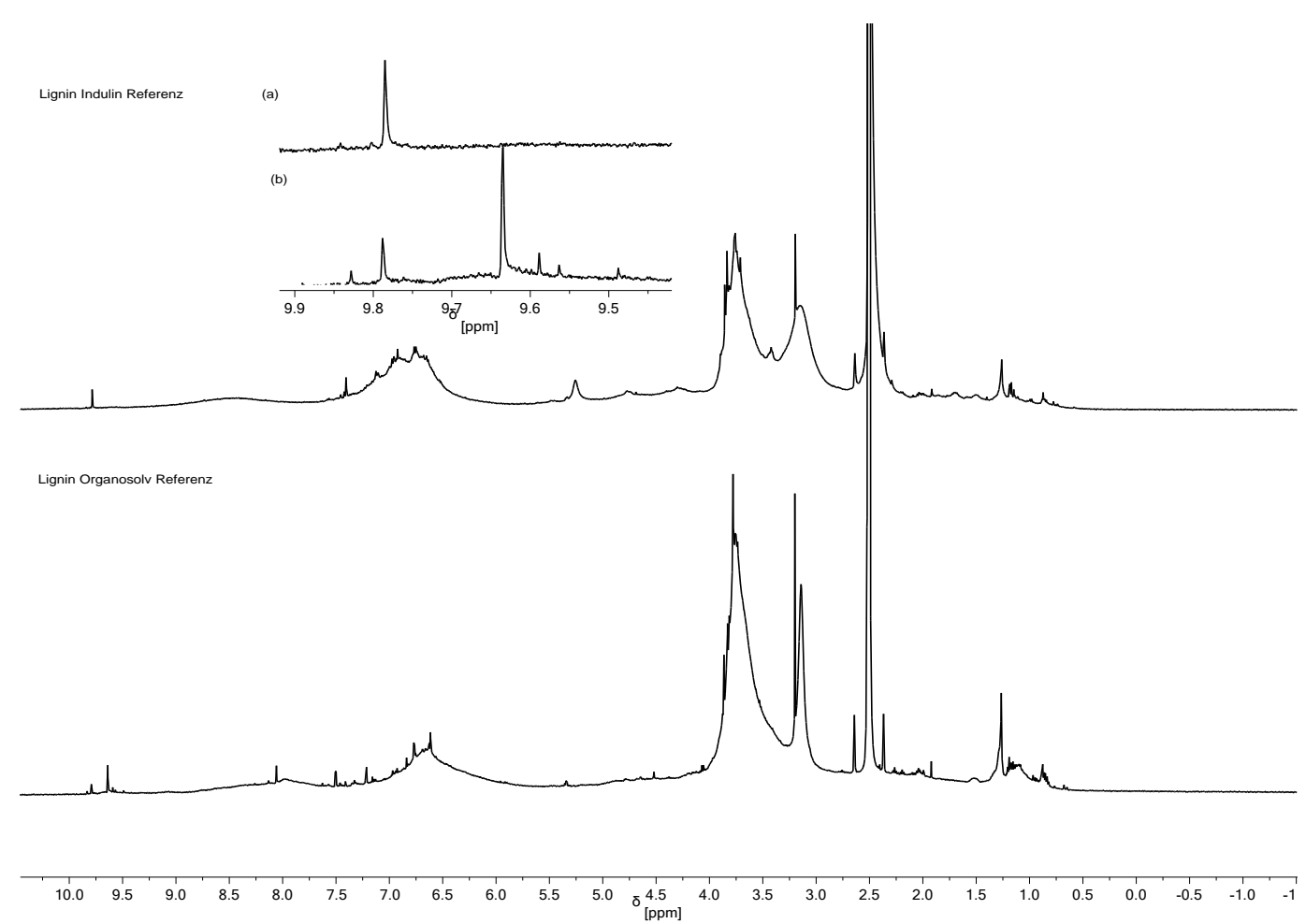

Abb. 3.6: ${ }^{1} \mathrm{H}-\mathrm{NMR}$ Spektren von Indulin Lignin (oben, (a)) und Organosolv Lignin (unten, (b)). Beide Proben sind Referenz Proben.

Bei der chemischen Verschiebung $\delta=9,63 \mathrm{ppm}$ befindet sich das H-Atom aus dem Zimtaldehyd nur im Organosolv Lignin und bei $\delta=9,79 \mathrm{ppm}$ ist das H-Atom des Benzaldehyds in beiden Ligninen deutlich zu erkennen. In dem Bereich zwischen 8,00 ppm und 8,13 ppm sind die H-Atome aus dem phenolischen $\mathrm{OH}-G r u p p e n$ nur im Organosolv $\mathrm{zu}$ finden. Der aromatische Bereich zwischen 6,40 ppm und 7,60 ppm ist für beide Lignins schwer zu interpretieren. Die genaue Zuordnung und die Analyse der Multiplizität des Signals sind nicht möglich. Zu derselben Folgerung ist Lundquist in seiner Arbeit ebenfalls gekommen. ${ }^{91}$

Die nächsten Unterschiede liegen im Bereich von 4,3 ppm bis 5,28 ppm. Hier befinden sich die Signale den $\mathrm{H}_{\alpha}$ bzw. $\mathrm{H}_{\beta}$ innerhalb von den $\beta-\mathrm{O}-4$ Bindungen. Im Schema 3.1 ist eine Modellkomponente mit den $\alpha, \beta$ und $\gamma$ Positionen im Lignin nach Lundquist dargestellt. ${ }^{90}$ 
Tab. 3.2: Chemische Verschiebung $\delta$ in $\mathrm{ppm}$ mit entsprechender Zuordnung zu ihrer Funktionalität für die ${ }^{1} \mathrm{H}-\mathrm{NMR}$ Spektren vom Lignin.

\begin{tabular}{|c|c|c|}
\hline$\delta[\mathrm{ppm}]$ & Funktionalität & Probe \\
\hline $0,75-2,20$ & $\begin{array}{c}\text { aliphatische } \\
\text { Seitenketten }\end{array}$ & Indulin / Organosolv \\
\hline 2,50 & DMSO-d 6 & Lösungsmittel \\
\hline $3,60-3,86$ & $\begin{array}{c}\mathrm{H} \text { in OCH} \text {-Gruppen } \\
\text { und } \mathrm{H} \text { in Alkohole }\end{array}$ & Indulin / Organosolv \\
\hline $4,30-4,80$ & $\mathrm{H}_{\beta}$ in $\beta-\mathrm{O}-4$ & Indulin \\
\hline 5,28 & $\mathrm{H}_{\alpha}$ in $\beta-\mathrm{O}-4$ & Indulin \\
\hline $6,40-7,60$ & $\mathrm{H}$ in Aromaten & Indulin / Organosolv \\
\hline $8,00-8,13$ & $\begin{array}{c}\mathrm{H} \text { in phen. OH- } \\
\text { Gruppen }\end{array}$ & Organosolv \\
\hline 9,63 & $\mathrm{H}$ in Zimtaldehyd & Organosolv \\
\hline 9,79 & $\mathrm{H}$ in Benzaldehyd & Indulin / Organosolv \\
\hline
\end{tabular}<smiles>[R]Oc1cc(C([R])C(CO)Oc2ccccc2OC)cc(OC)c1OC</smiles>

Schema 3.1: Modellkomponente mit den $\alpha, \beta$ und $\gamma$ Positionen im Lignin.

Diese Signale fehlen beim Spektrum vom Organosolv Lignin, weil während des Organosolv Produktionsprozesses die $\beta-\mathrm{O}-4$ Bindungen gebrochen werden (s. Kapitel 1). Im Schema 3.2 ist dieser Zerfall der $\beta-\mathrm{O}-4$ Bindung zu einer phenolischen Hydroxyl- bzw. einer CarbonylGruppen dargestellt. ${ }^{92}$

Bei der chemischen Verschiebung von 3,70 ppm bis 3,86 befindet sich ein sehr intensives und breites Signal von den Protonen aus den $\mathrm{OCH}_{3}-$ Gruppen in beiden Ligninen. Das Signal bei $\delta=3,3 \mathrm{ppm}$ charakterisiert das Wasser in der Probe. 
<smiles>[R]Oc1c(OC)cc(CC([R])Oc2c(OC)cc(CC([R])=O)cc2OC)cc1OC</smiles>

Schema 3.2: Zerfall der $\beta-\mathrm{O}-4$ Bindung zur phenolischen Hydroxyl- bzw. der CarbonylGruppe.

Es wurde festgestellt, die Proben mit der längeren Wartezeit bis zur NMR Messung haben den größeren Wasserpeak auf den Spektren von allen Lignin-Arten.

Der Lösungsmittelpeak von DMSO-d 6 befindet sich im Bereich bei der chemischen Verschiebung von 2,5 ppm. Die letzten Signale im Bereich zwischen 0,75 ppm und 2,20 ppm gehören zu aliphatischen Seitenketten.

\subsubsection{NMR Analyse des thermisch behandelten Indulin Lignins}

Das Indulin Lignin wird im Ofen bei $150{ }^{\circ} \mathrm{C}$ und $200{ }^{\circ} \mathrm{C}$ thermisch behandelt und durch die NMR Spektroskopie analysiert und mit der Referenz Probe verglichen.

In der Abb. 3.7 sind die ${ }^{1} \mathrm{H}-\mathrm{NMR}$ Spektren für das thermisch behandelte Indulin dargestellt. Die Zuordnung der chemischen Verschiebung ist in der Tab. 3.2 zu sehen.

Die $\mathrm{OCH}_{3}-G$ ruppen werden mit der steigenden Temperatur abgebaut. Deswegen sinkt die Intensität dieses Signales. Um diese Beobachtung zu überprüfen, wird das Integral des aromatischen Bereiches zwischen 6,50 ppm und 7,50 ppm von allen drei Spektren während der Auswertung auf eins gesetzt und das Integral zwischen 3,60 ppm und 3,90 ppm bestimmt. 


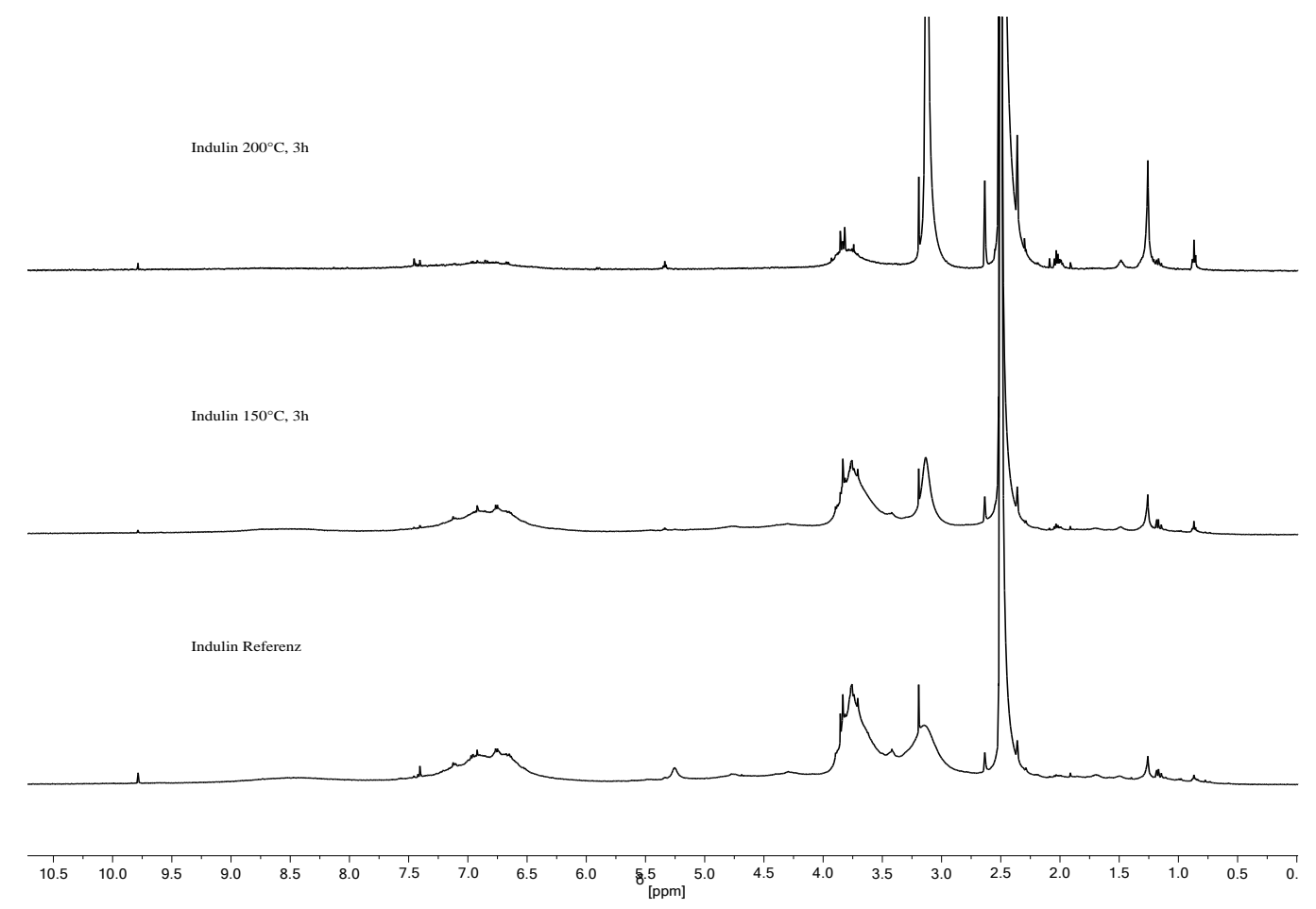

Abb. 3.7: Vergleich der ${ }^{1} \mathrm{H}-\mathrm{NMR}$ Spektren vom thermisch behandelten Indulin Lignin bei $200{ }^{\circ} \mathrm{C}$ (oben), $150{ }^{\circ} \mathrm{C}$ (in der Mitte) und der Referenz Probe (unten).

Aus diesen Daten lassen sich folgende Verhältnisse berechnen: Für die Referenzprobe ist der Wert 0,88; für das thermisch behandelte Indulin bei $150{ }^{\circ} \mathrm{C} 0,82$ und bei $200{ }^{\circ} \mathrm{C} \mathrm{0,44}$. Eine Erhöhung der Temperatur auf $150{ }^{\circ} \mathrm{C}$ führt zu einem leichten Abbau der $\mathrm{OCH}_{3}-$ Gruppen. Erst bei $200{ }^{\circ} \mathrm{C}$ werden die Protonen aus den $\mathrm{OCH}_{3}-$ Gruppen auf die Hälfte sinken.

Durch die ähnliche Methode wird auch das Verhältnis der $\mathrm{H}_{\alpha}$ Protonen in den $\beta$-O-4 Bindungen bei der chemischen Verschiebung zwischen $4,70 \mathrm{ppm}$ und $4,85 \mathrm{ppm}$ und der $\mathrm{H}_{\beta}$ in den $\beta$-O-4 Bindungen bei der chemischen Verschiebung zwischen 4,20 ppm und 4,35 ppm untersucht. Die Werte der Integralen der Referenzprobe und dem thermisch behandelten Indulin bei $150^{\circ} \mathrm{C}$ sind für $\mathrm{H}_{\alpha} 0,08$ und für $\mathrm{H}_{\beta} 0,10$. Daraus folgt, dass in diesen Temperaturbereich die $\beta$-O-4-Bindungen stabil sind und werden noch nicht zersetzt. Bei $200{ }^{\circ} \mathrm{C}$ sind diese Signale aber nicht mehr zu finden.

Entsprechend den Ergebnissen sind die $\beta$-O-4-Bindungen im Temperaturbereich bis $150{ }^{\circ} \mathrm{C}$ stabiler als $\mathrm{OCH}_{3}$-Gruppen. Mit der Erhöhung der Temperatur von $150{ }^{\circ} \mathrm{C}$ auf $200{ }^{\circ} \mathrm{C}$ werden die $\beta-\mathrm{O}-4$ Bindungen im Indulin fast komplett gebrochen und die Menge der $\mathrm{OCH}_{3}-$ Gruppen um die Hälfte reduziert. 


\subsubsection{NMR Analyse des thermisch behandelten Organosolv Lignins}

Das Organosolv Lignin wird im Ofen bei $150{ }^{\circ} \mathrm{C}$ und $200{ }^{\circ} \mathrm{C}$ thermisch behandelt, mit Hilfe von der ${ }^{1} \mathrm{H}-\mathrm{NMR}$ Spektroskopie charakterisiert und mit der Referenz Probe verglichen.

Die Abb. 3.8 zeigt ${ }^{1} \mathrm{H}$-NMR Spektren von der Referenz Probe und vom thermisch behandelten Organosolv.

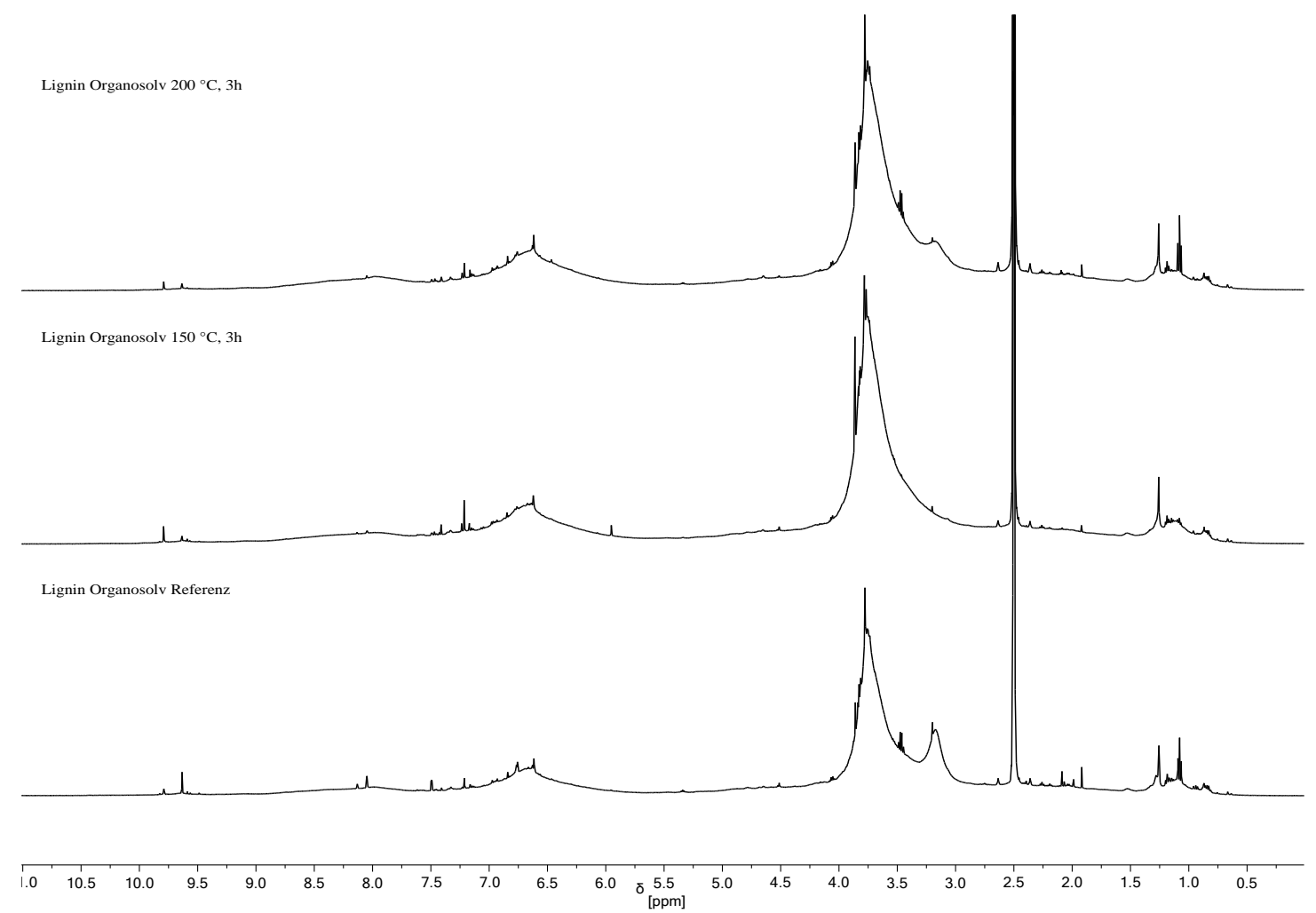

Abb. 3.8: Vergleich der ${ }^{1} \mathrm{H}-\mathrm{NMR}$ Spektren vom thermisch behandelten Organosolv Lignin bei $200{ }^{\circ} \mathrm{C}$ (oben), $150{ }^{\circ} \mathrm{C}$ (in der Mitte) und der Referenz Probe (unten).

Die Normierung ist in diesem Fall nicht möglich. Es wurden verschiedene Methoden ausprobiert, um die NMR Spektren zu normieren. Leider waren diese Ergebnisse nicht aussagekräftig. Deswegen werden die NMR Spektren qualitativ miteinander verglichen. Trotzdem lässt sich die 
wichtige Information aus dem Verhältnis der Integralen aus jedem Spektrum separat beobachten. Die Zuordnung der chemischen Verschiebung ist in der Tab. 3.2 eingegeben.

Mit der steigenden Temperatur wird die Intensität des Signals für das Proton aus dem Zimtaldehyd $(\delta=9,63 \mathrm{ppm})$ geringer. Bei $200^{\circ} \mathrm{C}$ wird auch der Peak für das Proton aus dem Benzaldehyd ( $\delta=9,79 \mathrm{ppm})$ kleiner. Die Signale der phenolischen OH-Gruppen mit der chemischen Verschiebung zwischen $8,00 \mathrm{ppm}$ und 8,13 ppm verlieren an der Intensität mit der steigenden Temperatur und bei $200{ }^{\circ} \mathrm{C}$ sind sie nicht mehr erkennbar.

\subsubsection{NMR Analyse des synthetisierten Lignin Modells aus dem Coniferylalkohol}

Das Lignin Modell wird aus dem Coniferylalkohol synthetisiert. Deswegen erfolgt zuerst die ${ }^{1} \mathrm{H}-\mathrm{NMR}$-Analyse der Ausgangssubstanz. Das entsprechende Spektrum ist in der Abb. 3.9 dargestellt.
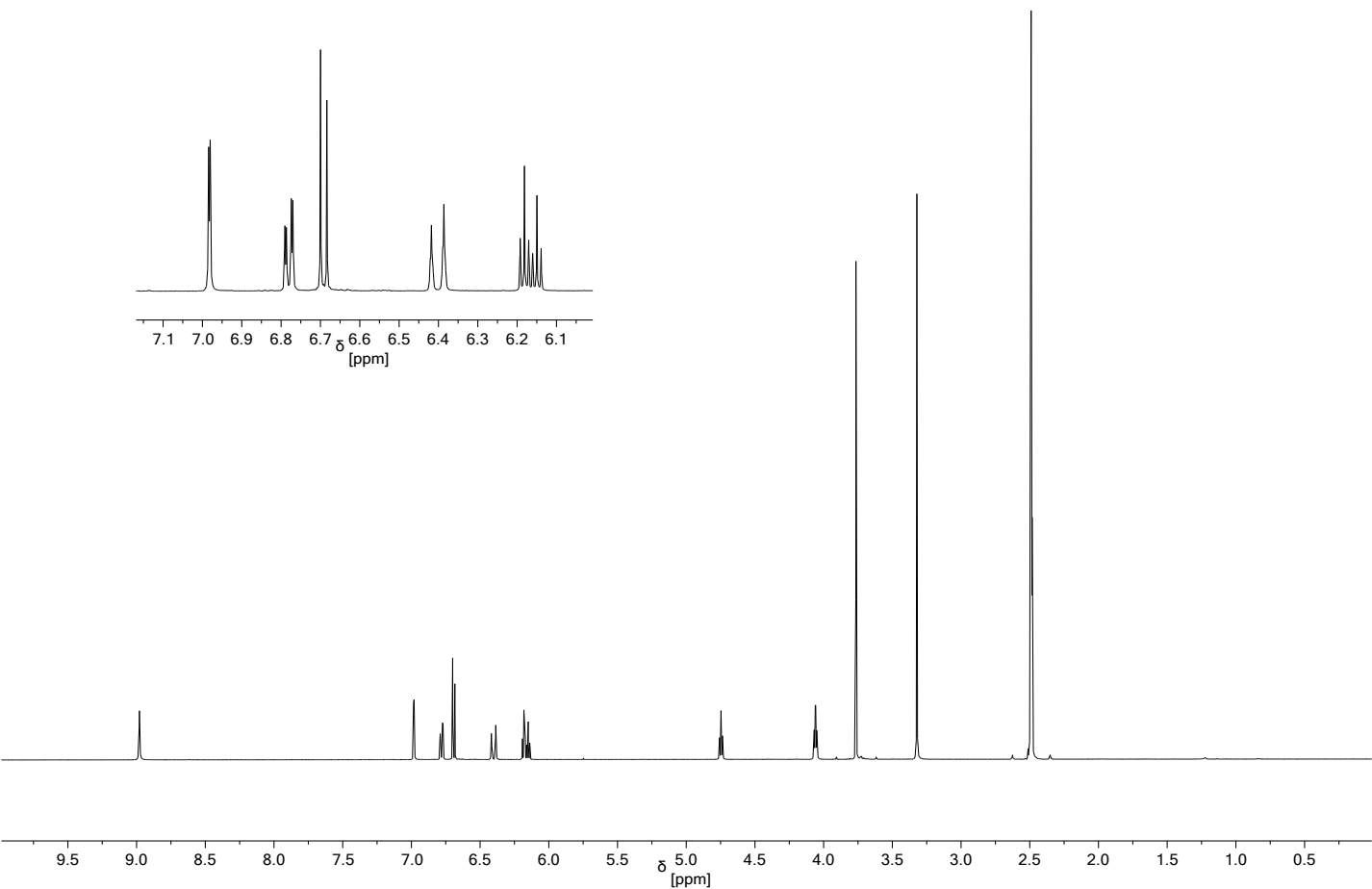

Abb. 3.9: ${ }^{1} \mathrm{H}-\mathrm{NMR}$ Spektrum vom Coniferylalkohol. 
Die Zuordnung der ${ }^{1} \mathrm{H}-\mathrm{NMR}$ Signale und die Anzahl der Protonen ist in der Tab. 3.3 eingegeben.

Tab. 3.3: Chemische Verschiebung $\delta$ in ppm der ${ }^{1} \mathrm{H}-\mathrm{NMR}$ Signale vom Coniferylalkohol.

\begin{tabular}{|c|c|c|}
\hline$\delta[\mathrm{ppm}]$ & $\begin{array}{c}\text { Anzahl der } \\
\text { H-Atomen }\end{array}$ & $\begin{array}{c}\text { Bezeichnung in } \\
\text { Schema 3.3 }\end{array}$ \\
\hline 3,77 & 3 & $\mathrm{a}$ \\
\hline $4,06-4,08$ & 2 & $\mathrm{~b}$ \\
\hline 4,76 & 1 & $\mathrm{c}$ \\
\hline $6,15-6,20$ & 1 & $\mathrm{~d}$ \\
\hline $6,40-6,43$ & 1 & $\mathrm{e}$ \\
\hline $6,69-6,71$ & 1 & $\mathrm{f}$ \\
\hline $6,78-6,80$ & 1 & $\mathrm{~g}$ \\
\hline 6,99 & 1 & $\mathrm{~h}$ \\
\hline 8,99 & 1 & $\mathrm{i}$ \\
\hline
\end{tabular}

Um die Interpretation der Protonen zu vereinfachen, ist die Struktur des Coniferylalkohols mit den entsprechenden Protonen im Schema 3.3 gezeichnet.<smiles>COc1cc(/C=C/CO)ccc1O</smiles>

Schema 3.3: Struktur des Coniferylalkohols mit den bezeichneten Protonen aus der Tabelle 3.3.

Zuerst wird das synthetisierte Lignin Modell im Ofen bei $150{ }^{\circ} \mathrm{C}$ und $200{ }^{\circ} \mathrm{C}$ erhitzt. Danach werden die thermisch behandelten Proben und die Referenz Probe in der Lösung DMSO-d6 gelöst und mithilfe von der ${ }^{1} \mathrm{H}-\mathrm{NMR}$ Spektroskopie analysiert. Aufgrund der niedrigen Löslichkeit der Probe, die bei $200{ }^{\circ} \mathrm{C}$ thermisch behandelt wurde, konnten keine Daten ermittelt werden. In der Abb. 3.10 sind die ${ }^{1} \mathrm{H}-\mathrm{NMR}$ Spektren der Referenz Probe und des Lignin Modells dargestellt, die bei $150{ }^{\circ} \mathrm{C}$ erhitzt wurde. 


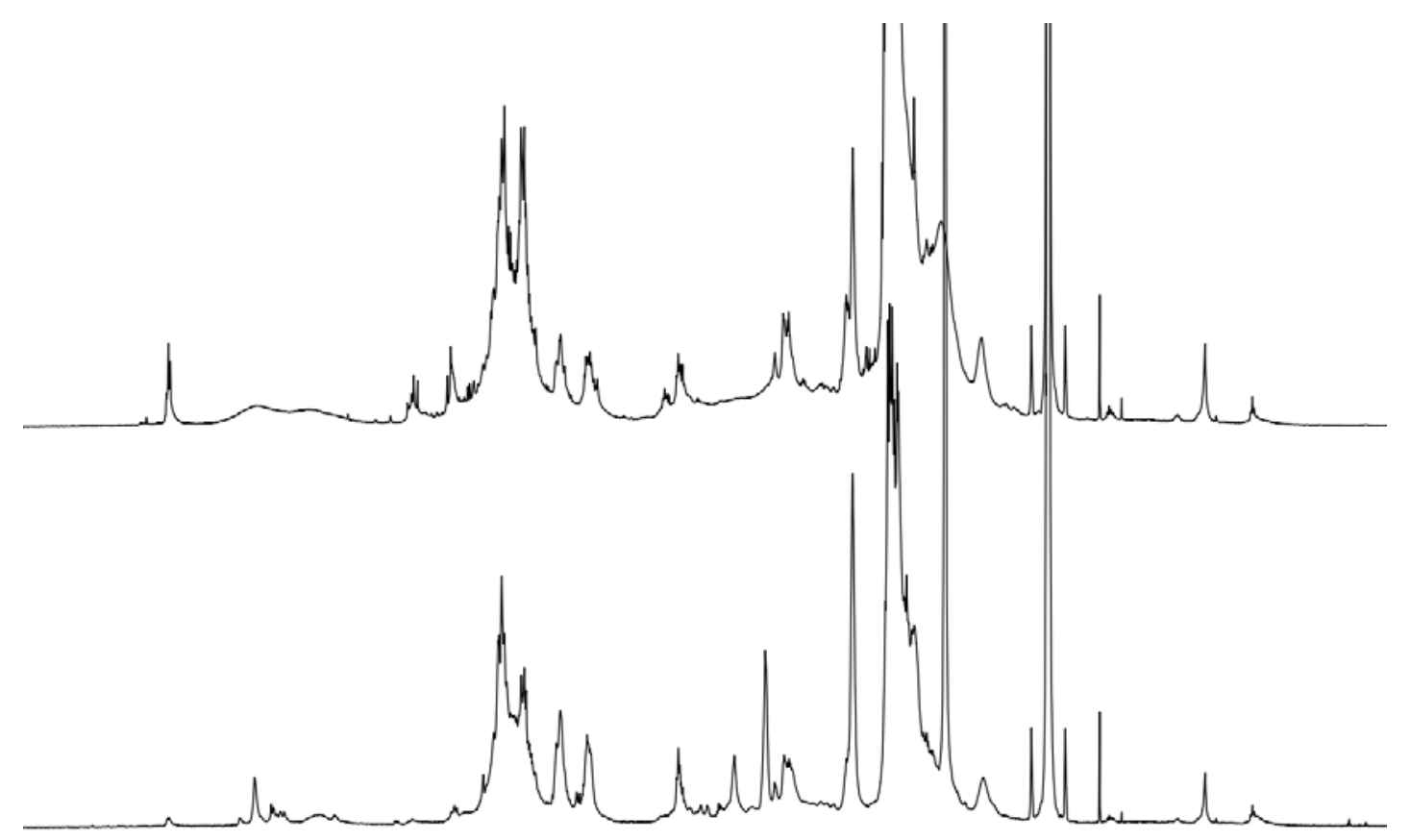

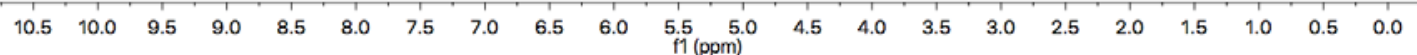

Abb. 3.10: Vergleich der ${ }^{1} \mathrm{H}-\mathrm{NMR}$ Spektren des Lignin Modells, das bei $150{ }^{\circ} \mathrm{C}$ thermisch behandelt wurde (oben), mit der Referenz Probe (unten).

Beim Vergleich der Spektren des Lignin Modells und des Coniferylalkohols ist eine deutliche Zunahme der Intensität für den Aromatischen Bereich von 6,5 ppm bis 7,0 ppm und für die $\mathrm{OCH}_{3}-$ Gruppen zwischen 3,6 ppm und 3,8 ppm zu sehen. Das Signal des $\mathrm{H}$-Atoms aus dem Aldehyd mit der $\delta$ von 9,58 ppm ist neu im Lignin Modell und in NMR Spektren vom Coniferylalkohol fehlt, genauso wie die Signale von den aliphatischen Seitenketten bei der $\delta$ zwischen $0,75 \mathrm{ppm}$ und 2,20 ppm. Allgemein hat ${ }^{1} \mathrm{H}-\mathrm{NMR}$ Spektrum des Lignin Modells eine Ähnlichkeit mit dem Spektrum vom Indulin Lignin (s. Abb. 3.6). Um die NMR Spektren der Referenz Probe und des thermisch behandelten Lignin Modell zu vergleichen, wird die ähnliche Normierungsmethode ausgesucht wie für das Indulin Lignin. In diesem Fall wird das Integral des Signals vom Proton aus dem Aldehyd auf eins normiert. Mit der steigenden Temperatur nimmt das Integral des Signals von den $\mathrm{OCH}_{3}$-Gruppen von 60,59 auf 29,44 ab. Gleicher Trend wird im Indulin Lignin beobachtet. Ebenfalls bleiben die $\beta$-O-4-Bindungen bei der $\delta$ zwischen $4,48 \mathrm{ppm}$ und $4,65 \mathrm{ppm}$ bei $150^{\circ} \mathrm{C}$ stabil. Gleichzeitig 
werden die H-Protonen aus den Alkoholen mit $\delta 4,76 \mathrm{ppm}$ komplett zersetzt. Die phenolische Protonen zwischen 8,7 ppm und 8,86 ppm werden mit der steigenden Temperatur kaum beobachtet.

Die NMR Spektren bestätigen, dass die Synthese des Lignin Modells erfolgreich ist.

\subsection{Gelpermeationschromatographie (GPC) des Lignins}

Die Molmasse des Lignins wird mithilfe von GPC bestimmt. Um die Messungen durchzuführen, sollen die untersuchten Proben in THF gelöst werden. Wegen der niedrigen Löslichkeit des Indulin Lignins, was bei $200{ }^{\circ} \mathrm{C}$ erhitzt wurde, und des Lignin Modells fehlen einige Ergebnisse.

In der Tabelle 3.4 sind die Ergebnisse der GPC Analyse mit der Lösungsmitteln THF eingegeben, wobei $\bar{M}_{\mathrm{n}}$ der Mittelwert des Zahlenmittels, $\bar{M}_{\mathrm{w}}$ der Mittelwert des Gewichtsmittels und $Đ$ die Polydispersität sind.

Tab. 3.4: GPC Ergebnisse für das Lignin mit THF als Eluent.

\begin{tabular}{|c|c|c|c|}
\hline Probe & $\begin{array}{c}\bar{M}_{\mathrm{n}} \\
{\left[\mathrm{g} \cdot \mathrm{mol}^{-1}\right]}\end{array}$ & $\begin{array}{c}\bar{M}_{\mathrm{w}} \\
{\left[\mathrm{g} \cdot \mathrm{mol}^{-1}\right]}\end{array}$ & Đ \\
\hline Organosolv Referenz & 1278 & 2113 & 1,65 \\
\hline Organosolv $150 \mathrm{C}$ & 1515 & 3060 & 2,02 \\
\hline Organosolv $200{ }^{\circ} \mathrm{C}$ & 1820 & 7435 & 4,08 \\
\hline Indulin Referenz & 751 & 1083 & 1,44 \\
\hline Indulin $150{ }^{\circ} \mathrm{C}$ & 883 & 1211 & 1,37 \\
\hline
\end{tabular}

Die Polydispersität ist die Breite der Massenverteilung und wird nach der Gleichung 3.1 berechnet.

$$
\mathrm{Ð}=\frac{\bar{M} w}{\bar{M} n}
$$


Da einige Proben sich sehr schlecht in THF lösen, wird noch eine andere Lösung für die GPC verwendet. Dafür wurde DMAc ausgesucht, indem die Löslichkeit des Lignins deutlich besser ist. Ausnahme ist das Indulin Lignin, was bei $200^{\circ} \mathrm{C}$ erhitzt wurde. Hier kann die Probe nicht vollständig gelöst werden. Die GPC Werte des Lignins mit DMAc als Eluent sind in der Tabelle 3.5 eingegeben.

Tab. 3.5: GPC Ergebnisse für das Lignin mit DMAc als Eluent.

\begin{tabular}{|c|c|c|c|}
\hline Probe & $\begin{array}{c}\overline{M_{\mathrm{n}}} \\
{\left[\mathrm{g} \cdot \mathrm{mol}^{-1}\right]}\end{array}$ & $\begin{array}{c}\overline{M_{\mathrm{w}}} \\
{\left[\mathrm{g} \cdot \mathrm{mol}^{-1}\right]}\end{array}$ & Đ \\
\hline Organosolv Referenz & 1779 & 4269 & 2,40 \\
\hline Organosolv $150 \mathrm{C}$ & 2674 & 9617 & 3,60 \\
\hline Organosolv $200{ }^{\circ} \mathrm{C}$ & 5152 & 7058 & 1,37 \\
\hline Indulin Referenz & 9989 & 17950 & 1,80 \\
\hline Indulin $150{ }^{\circ} \mathrm{C}$ & 15820 & 31820 & 2,01 \\
\hline Indulin $200^{\circ} \mathrm{C}$ & 9571 & 3642 & 3,81 \\
\hline Lignin Modell Referenz & 1709 & 6842 & 4,00 \\
\hline Lignin Modell $150{ }^{\circ} \mathrm{C}$ & 2587 & 17260 & 6,67 \\
\hline
\end{tabular}

Beim Vergleich der Werte aus der Tabelle 3.4 und 3.5 wird eindeutig, dass die Molmasse der Proben, in denen DMAc als Eluent ist, größer ist als bei THF. Es liegt daran, dass sich das Lignin in DMAc besser lösen kann als in THF. Erst die Seitenketten und andere kleinere Lignin-Anteile werden in THF gelöst und durch GPC entsprechend analysiert. Wohingegen in DMAc lassen sich deutlich größere Lignin-Anteile lösen.

Dabei ist zu erkennen, dass die molare Masse mit der steigenden Temperatur permanent größer wird. Dies kann dadurch erklärt werden, dass die durch die Hitze entstehende Bindungsbrechung zu den neuen intramolekularen Verknüpfungen in der Struktur führen kann. Neue gebildete Verknüpfungen, wie z.B. die C-C-Bindungen zwischen den Lignineinheiten, sind stabiler als die zuvor vorhandenen. ${ }^{93}$ Dadurch wird das Ligninpolymer weiter vergrößert. Die Ausnahme ist der Wert des Indulin Lignins, was bei $200{ }^{\circ} \mathrm{C}$ erhitzt wurde, weil dies nicht vollständig gelöst werden kann, auch nicht in DMAc.

Die GPC Werte für das Lignin Modell zeigen mit der steigenden Temperatur denselben Trend - die Molmasse der Probe wird bei der Erhitzung größer. 
Diese Ergebnisse bestätigen ebenso die erfolgreiche Synthese des Lignin Modells aus dem Coniferylalkohol. Zum Vergleich, die Molmasse des Coniferylalkohols liegt bei $180,20 \mathrm{~g} \cdot \mathrm{mol}^{-1}$.

\subsection{Dynamische Differenzkalorimetrie (DSC) des Lignins}

Die DSC Messungen werden durchgeführt, um eine thermische Charakterisierung der drei Lignin-Arten $\mathrm{zu}$ verdeutlichen. In den Tabellen 3.6-3.8 sind die Glasübergangstemperaturen des technischen Lignins, dem extrahierten Lignin und dem synthetisierten Lignin Modell entsprechend dargestellt.

Die Glasübergangstemperaturen des thermisch behandelten Organosolvs und der Referenz Probe sind relativ stabil und liegen bei ca. $153{ }^{\circ} \mathrm{C}$ (s. Tabelle 3.6). Die unveränderten $T_{\mathrm{g}}$ Werte vom Organosolv können durch das Produktionsverfahren erklärt werden. Während des Organosolv Prozesses wird die Struktur des Lignins erheblich zerstört. Hierdurch bewirkt auch die thermische Behandlung in dem Temperaturbereich von $150{ }^{\circ} \mathrm{C}$ bis $220^{\circ} \mathrm{C}$ danach keinen signifikanten Veränderungen auf die Glasübergangstemperatur.

Im Gegensatz zum Organosolv wird mit der steigenden thermischen Behandlung die Glasübergangstemperatur vom Indulin größer (s. Tabelle 3.6). Diese Ergebnisse unterstützen die Aussage, die oben im Abs. 3.4 getroffen wurde - die durch die Hitze entstehende Bindungsbrechung führt zu den neuen intramolekularen Verknüpfungen in der Struktur. Infolgedessen wird die Vernetzung im Lignin wachsen und die Glasübergangstemperatur dementsprechend steigen. 
Tab. 3.6: Die Glasübergangstemperatur $T_{g}$ in ${ }^{\circ} \mathrm{C}$ für das technische Lignin Indulin und Organosolv.

\begin{tabular}{|c|c|c|}
\hline \multirow{2}{*}{$\begin{array}{c}\text { Bedingungen der thermischen } \\
\text { Behandlung }\end{array}$} & Indulin & Oragnosolv \\
\cline { 2 - 3 } & $T_{\mathrm{g}}\left[{ }^{\circ} \mathrm{C}\right]$ & $T_{\mathrm{g}}\left[{ }^{\circ} \mathrm{C}\right]$ \\
\hline Referenz & 163 & 153 \\
\hline $150^{\circ} \mathrm{C}$ & 172 & 153 \\
\hline $200^{\circ} \mathrm{C}$ & 219 & 147 \\
\hline $220^{\circ} \mathrm{C}$ & $218 / 245$ & 156 \\
\hline
\end{tabular}

Die DSC Werte vom extrahierten aus der TM Buche bzw. aus der TM Kiefer Lignin zeigen erwartete Glasübergangstemperaturen (s. Tabelle 3.7). Diese Werte sind relativ konstant und befinden im Temperaturbereich bei ca. $153^{\circ} \mathrm{C}$. Auch hier wird es durch den Gewinnungsprozess erklärt. Die Behandlung mit der konzentrierten Säure zerstört die Struktur des Lignins sehr stark, sodass die thermische Behandlung bis $220^{\circ} \mathrm{C}$ keine Veränderungen auf die Glasübergangstemperatur verursachen kann. Ausnahme ist der Wert des extrahierten Lignins aus der Referenz Buche, der bei $189^{\circ} \mathrm{C}$ liegt. Der Wert ist fehlerhaft. Wahrscheinlich waren einige Polysaccharide im Lignin beim Gewinnungsprozess vorhanden.

Tab. 3.7: Die Glasübergangstemperatur $T_{\mathrm{g}}$ in ${ }^{\circ} \mathrm{C}$ für das extrahierte aus der TM Kiefer bzw. aus der TM Buche Lignin.

\begin{tabular}{|c|c|c|}
\hline Modifikationstemperatur & $\begin{array}{c}\text { extrah. Lignin } \\
\text { aus der Kiefer }\end{array}$ & $\begin{array}{c}\text { extrah. Lignin } \\
\text { aus der Buche }\end{array}$ \\
\cline { 2 - 3 } & $T_{\mathrm{g}}\left[{ }^{\circ} \mathrm{C}\right]$ & $T_{\mathrm{g}}\left[{ }^{\circ} \mathrm{C}\right]$ \\
\hline Referenz & 153 & 189 \\
\hline $180^{\circ} \mathrm{C}$ & 152 & 152 \\
\hline $200^{\circ} \mathrm{C}$ & 153 & 153 \\
\hline $220^{\circ} \mathrm{C}$ & 153 & 153 \\
\hline
\end{tabular}

Die Glasübergangstemperatur des synthetisierten Lignin Modells (s. Tabelle 3.8) ist fast genauso hoch wie für die anderen Lignin-Sorten. 
Tab. 3.8: Die Glasübergangstemperatur $T_{\mathrm{g}}$ in ${ }^{\circ} \mathrm{C}$ für das synthetisierte Lignin Modell.

\begin{tabular}{|c|c|}
\hline Bedingungen der thermischen & Lignin Modell \\
\cline { 2 - 2 } Behandlung & $T_{\mathrm{g}}\left[{ }^{\circ} \mathrm{C}\right]$ \\
\hline Referenz & 149 \\
\hline $150{ }^{\circ} \mathrm{C}$ & 153 \\
\hline $200^{\circ} \mathrm{C}$ & 178 \\
\hline
\end{tabular}

Dies bekräftigt wieder, dass die Synthese des Lignin Modells erfolgreich war und die Vernetzung im synthetisierten Lignin ähnlich stark wie in technischem oder extrahiertem Lignin ist. Auch in diesem Fall führt die thermische Behandlung zu den neuen intramolekularen Verknüpfungen, was automatisch zur Steigung der Glasübergangstemperatur führt.

\subsection{Massenverlust des Lignins nach der thermischen Behandlung}

Nach der Bestimmung der Molmasse und der Glasübergangstemperatur des Lignins wird auch der Massenverlust (ML, englisch Mass Loss) nach der thermischen Behandlung vom technischen Lignin untersucht. Der Massenverlust wird nach der Gleichung 6.1 berechnet. Die Messergebnisse sind in der Tabelle 3.9 eingetragen.

Tab. 3.9: Die Werte des Massenverlusts des technischen Lignins in \% nach der thermischen Behandlung im Ofen bei $150{ }^{\circ} \mathrm{C}$ und $200^{\circ} \mathrm{C}$.

\begin{tabular}{|c|c|c|}
\hline Probe & $\begin{array}{c}\text { Bedingungen der } \\
\text { thermischen } \\
\text { Behandlung }\end{array}$ & ML [\%] \\
\hline \multirow{3}{*}{ Indulin } & $150{ }^{\circ} \mathrm{C}$ & 5,3 \\
\cline { 2 - 3 } & $200^{\circ} \mathrm{C}$ & 8,4 \\
\cline { 2 - 3 } & $220^{\circ} \mathrm{C}$ & 10,5 \\
\hline \multirow{5}{*}{ Organosolv } & $150{ }^{\circ} \mathrm{C}$ & 3,0 \\
\cline { 2 - 3 } & $200{ }^{\circ} \mathrm{C}$ & 4,5 \\
\cline { 2 - 3 } & $220^{\circ} \mathrm{C}$ & 9,6 \\
\hline
\end{tabular}


Wie erwartet steigt der Massenverlust des Lignins mit der Erhöhung der Behandlungstemperatur hauptsächlich durch den Verlust von den flüchtigen Stoffen, dem gebundenen Wasser und durch die Zersetzung der Lignineinheiten. ${ }^{94}$

Interessanterweise wird der Massenverlust vom Indulin größer als vom Organosolv. Diese Ergebnisse widersprechen den Ergebnissen des Massenverlustes des Holzes, da die Buche einen größeren Massenverlust als die Kiefer hat. Dieses passiert aufgrund der Zersetzung der anderen Holzbestandteile, wie z. B. Hemicellulose oder Cellulose.

Der Massenverlust des puren Lignins bestätigt die im Kapitel 2 genannte Hypothese, dass Lignin aus der Kiefer im Temperaturbereich bei $200{ }^{\circ} \mathrm{C}$ sich schneller zersetzt als das Lignin aus der Buche.

\subsection{Massenspektrometrie (MS)}

Die Massenspektren des Lignins werden mit zwei unterschiedlichen Spektrometern aufgenommen: mit einem Elektrospray-Ionisation MAXIS-Massenspektrometer (ESI-QTOF-MS) und einem AUTOFLEX SPEED Massenspektrometer (MALDI-TOF), um die bessere Methode zu finden. Der Messbereich liegt zwischen $50 \mathrm{~m} / \mathrm{z}$ und $1650 \mathrm{~m} / \mathrm{z}$. Aus den Messreihen wird deutlich, dass die ESI-negativ Methode am besten passt, weil diese zu intensiven Signalen in den Spektren führt. Eine Zuordnung von den Signalen ist nur möglich im Bereich bis $m / z<400$. Dies wird dadurch erklärt, dass die Struktur des Lignins sehr komplex ist und nur die Fragmente mit der niedrigen Molekülzahl leicht identifizierbar sind. Dazu gehören Fragmente mit folgenden chemischen Formeln: $\mathrm{C}_{20} \mathrm{H}_{28} \mathrm{O}_{2}$ mit $m / z=299,20 ; \mathrm{C}_{20} \mathrm{H}_{30} \mathrm{O}_{2}$ mit $m / z=301,22 ; \mathrm{C}_{20} \mathrm{H}_{28} \mathrm{O}_{3}$ mit $m / z=315,20$ und $\mathrm{C}_{20} \mathrm{H}_{30} \mathrm{O}_{3}$ mit $m / z=317,21$. Wohingegen hochmolekulare Fragmente die gleiche Masse haben können, aber eine unterschiedliche Struktur. Dies macht die MS Analyse kompliziert oder fast unmöglich. Zu den Nachteilen der Methode gehört auch die schwierige Löslichkeit des Lignins, deswegen können nicht alle Proben analysiert werden. MALDI-MS als alternative Methode passt dafür besser, weil die Proben auch als Festkörper gemessen können. In diesem Fall werden die Signale vorwiegend im höheren Bereich mit $m / z>1200$ beobachtet.

Aus den beiden Methoden wurden ähnliche Ergebnisse ermittelt. Mit der 
steigenden Behandlungstemperatur wird eine Zunahme der Signale im höheren Bereich von $\mathrm{m} / \mathrm{z}$ beobachtet. Dies bestätigt die GPC- und DSCErgebnisse, dass die Molmasse mit der Erhöhung der Behandlungstemperatur größer wird.

\subsection{Elektronenspinresonanz Spektroskopie (ESR) des Lignins}

Zum Schluss wird die Radikalkonzentration für die drei Ligninarten mithilfe von Elektronenspinresonanz Spektroskopie untersucht. Alle Proben werden als Festproben gemessen. Die Auswertungsmethode ist im Kapitel 2 deutlich beschrieben. Für alle ESR Messungen wird der $g$-Faktor nach der Gleichung 2.1 berechnet. Die ESR Signale von allen Proben befinden sich auf einem Resonanzfeld mit dem $g$-Faktor von ca. 2,0023. Dieser Wert entspricht dem $g$-Faktor für die organischen freien Radikale. ${ }^{95}$ In der Tabelle 3.10 sind die ESR Messergebnisse des technischen thermisch behandelten Lignins dargestellt, wobei das Integral des Absorptionssignals proportional zur der Radikalkonzentration in der Probe ist. ${ }^{96}$

Sowohl Indulin Referenz als auch Organosolv Referenz enthalten eine hohe Radikalkonzentration auch ohne thermische Behandlung. Einerseits wird es durch den Gewinnungsprozess des Lignins erklärt, weil viele Bindungen während der Herstellung gebrochen werden, andereseits wird es durch die Biosynthese des Lignins erklärt. ${ }^{97}$ Genau wie für nicht modifizierten Holzproben (s. Kapitel 2).

Der gleiche Trend wie bei dem thermisch modifizierten Holz zeigt auch das thermisch behandelte Lignin - mit der steigenden Behandlungstemperatur vergrößert sich die Anzahl der freien stabilen Radikalen (s. Tab. 3.10). Währenddessen ist die freie Radikalkonzentration vom Indulin größer als vom Organosolv. Dies widerspricht den Ergebnissen vom Holz, in denen die Radikalkonzentration nach der thermischen Modifikation in der Buche deutlich größer als in der Kiefer ist (s. Kapitel 2). Es liegt daran, dass die Struktur vom Organosolv schon während der Produktion stark zersetzt wird und keine signifikanten Veränderungen in der Struktur unter der Hitze bis $220^{\circ} \mathrm{C}$ vorkommen können. Die GPC- und DSC-Analyse bestätigt diese Aussage eindeutig. 
Tab. 3.10: Die Radikalkonzentration vom thermisch behandelten Indulin bzw. Organosolv und von den entsprechenden Referenz Proben.

\begin{tabular}{|c|c|c|}
\hline \multirow{2}{*}{$\begin{array}{c}\text { Bedingungen der thermischen } \\
\text { Behandlung }\end{array}$} & \multicolumn{2}{|c|}{ Integral des Absorptionssignals } \\
\cline { 2 - 3 } & Indulin & Organosolv \\
\hline Referenz & $2135 \pm 22$ & $1947 \pm 18$ \\
\hline $150^{\circ} \mathrm{C}$ & $3713 \pm 30$ & $3419 \pm 26$ \\
\hline $200^{\circ} \mathrm{C}$ & $4580 \pm 35$ & $3492 \pm 9$ \\
\hline $220^{\circ} \mathrm{C}$ & $5793 \pm 27$ & $3759 \pm 11$ \\
\hline
\end{tabular}

In der Abb. 3.11 sind die doppelten Integrale der ESR Signalen vom extrahierten aus der TM Kiefer (schwarz) bzw. aus der TM Buche (rot) dargestellt. Für diesen Fall ist auch derselbe Trend zu erkennen - die Radikalkonzentration in den Proben steigt mit der Erhöhung der Modifikationstemperatur. Auch hier ist die Anzahl der freien stabilen Radikalen im Lignin aus der Kiefer größer als aus der Buche. Die mögliche Erklärung ist die starke Vernetzung, die freie Radikale stabilisiert. 


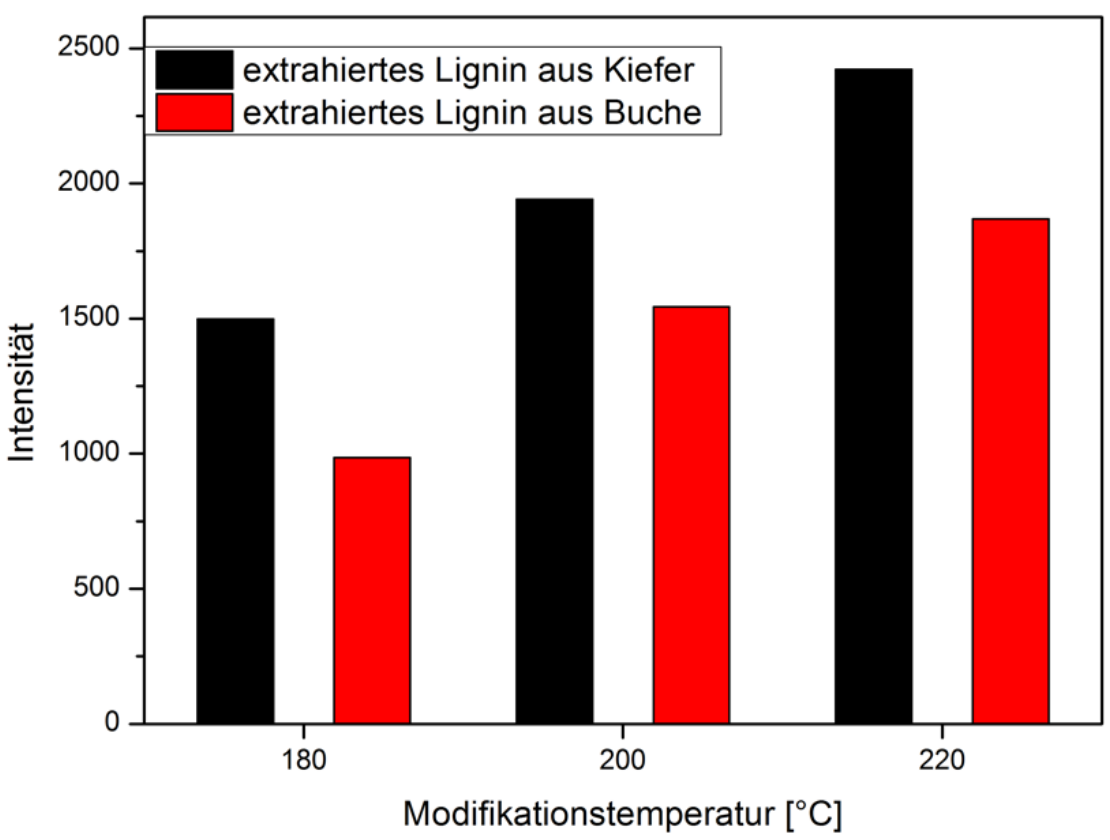

Abb. 3.11: Das doppelte Integral des ESR Signals gegen die Modifikationstemperatur in ${ }^{\circ} \mathrm{C}$ vom extrahierten aus der TM Kiefer (schwarz) bzw. aus der TM Buche (rot) Lignin.

In der Tabelle 3.11 sind die ESR Ergebnisse vom thermisch behandelten Lignin Modell dargestellt. Diese Werte sind besonders interessant, weil der Trend hier in die entgegengesetzte Richtung verläuft als beim TMT oder beim thermisch behandelten Lignin. Mit der Steigerung der Behandlungstemperatur sinkt die Radikalkonzentration in der Probe.

Tab. 3.11: Die Radikalkonzentration vom thermisch behandelten Lignin Modell und der Referenz Probe.

\begin{tabular}{|c|c|}
\hline Probe & Integral des Absorptionssignals \\
\hline Referenz Lignin Modell & $401 \pm 15$ \\
\hline $150^{\circ} \mathrm{C}$ & $147 \pm 3$ \\
\hline $200^{\circ} \mathrm{C}$ & $129 \pm 5$ \\
\hline
\end{tabular}

Dies wird dadurch erklärt, dass die Struktur des Lignin Modells geringeren Polymerisationsgrad hat als das Holz oder das technische Lignin. Deswegen werden freie Radikaler schneller gekoppelt. Das Lignin Modell wird nur aus einem 
Baustein synthetisiert, nur aus dem Coniferylalkohol. Das vereinfachte Lignin Modell bietet keine große Stabilität für die freien Radikalen. Deswegen werden die Elektronen durch die konjugierte Struktur kaum delokalisiert oder die Beweglichkeit der Lignineinheiten stärker ist als im Holz und im technischen Lignin, sodass die freie Radikale miteinander abreagieren. 


\subsection{Fazit}

In diesem Kapitel wurden drei Ligninarten untersucht: das technische Lignin (Indulin und Organosolv), das extrahierte aus der TM Buche und der TM Kiefer Lignin und das aus dem Coniferylalkohol synthetisierte Lignin Modell. Alle Ligninarten wurden thermisch im Ofen im Temperaturbereich von $150{ }^{\circ} \mathrm{C}$ bis $200{ }^{\circ} \mathrm{C}$ behandelt und mithilfe von den unterschiedlichen Methoden analysiert und mit den Referenzproben verglichen, um die Veränderungen in der molekularen Ligninstruktur zu charakterisieren, die durch Hitze entstehen.

${ }^{1} \mathrm{H}-\mathrm{NMR}$ und IR Analyse waren sehr informativ. Mit der Steigerung der Behandlungstemperatur sinkt der Anteil von den $\mathrm{OCH}_{3}-\mathrm{Gruppen}_{\mathrm{im}}$ Indulin. Im Temperaturbereich bis $150^{\circ} \mathrm{C}$ passiert es sehr langsam, aber von $150{ }^{\circ} \mathrm{C}$ bis $200{ }^{\circ} \mathrm{C}$ wird der Anteil von den $\mathrm{OCH}_{3}$-Gruppen um die Hälfte weniger als bei der Referenz Probe. Die $\beta$-O-4-Bindungen sind bis $150{ }^{\circ} \mathrm{C}$ relativ stabil im Indulin, aber bei $200{ }^{\circ} \mathrm{C}$ sind die Bindungen fast komplett gebrochen. Für das Organosolv zeigen die ${ }^{1} \mathrm{H}-\mathrm{NMR}$ Spektren, dass die Protonen aus dem Zimtaldehyd mit der steigenden Behandlungstemperatur abgebaut werden. Bei $200^{\circ} \mathrm{C}$ sinkt auch der Peak vom H-Atom aus dem Benzaldehyd. Die phenolische OH-Gruppen werden unter der Hitze auch abgebaut und bei $200^{\circ} \mathrm{C}$ sind sie fast nicht mehr erkennbar. Für das Lignin Modell haben NMR Spektren bestätigt, dass die Synthese aus dem Coniferylalkohol erfolgreich war und die Struktur des Lignin Modells liegt sehr nah zur Struktur des Indulins. Mit der Steigerung der Behandlungstemperatur werden auch die $\mathrm{OCH}_{3}-\mathrm{Gruppen}$, die phenolische und aliphatische $\mathrm{OH}-\mathrm{Gruppen}$ abgebaut. Die $\beta$-O-4-Bindungen sind ähnlich wie im Lignin bis $150^{\circ} \mathrm{C}$ stabil, danach zersetzten sie sich unter der Hitze.

Die DSC, GPC und die MS bestätigen, dass sich die neuen intramolekularen Verknüpfungen unter der Hitze bilden. Dadurch wird die Molmasse und der Vernetzungsgrad größer als in der Referenz Probe.

Der Massenverlust steigt unter der Hitze. Unerwartet war, dass ML vom Indulin höher als vom Organosolv ist, weil im TM Holz der Trend in Gegenrichtung verläuft. Der Unterschied wurde durch andere Holzkomponenten erklärt. 


\section{Kapitel 4 Behandlung des TMT Pulvers durch eine radikalische Polymerisation}

Die folgenden Abschnitte behandeln verschiedene Möglichkeiten, um die freien Radikale in TMT nützlich zu machen und weiter in einer freien radikalischen Polymerisation trotz ihrer hohen Stabilität anzuwenden. Es wird TMT Pulver mit unterschiedlichen Modifikationsparametern ( $T_{\mathrm{mod}}$ : $180^{\circ} \mathrm{C}, 200^{\circ} \mathrm{C}$ und $220^{\circ} \mathrm{C}, p_{\text {mod }} 5$ bar, Holzart: Kiefer, Buche) in einer radikalischen Polymerisation in Substanz und im Lösungsmittel verwendet. Im Anschluss daran werden die Analyseergebnisse vorgestellt.

\subsection{Radikalische Polymerisation des TMT Pulvers in der Substanz ohne Initiator}

Die radikalische Polymerisationsreaktion beginnt mit einem radikalerzeugenden Schritt mit Hilfe eines Initiators. ${ }^{98}$ Da TMT eine hohe Konzentration der freien Radikalen enthält (s. Kapitel 2), wird die radikalische Polymerisation direkt mit dem Kettenwachstumsschritt gestartet, um den Initiator zu vermeiden.

Schema 4.1 zeigt eine schematische Darstellung der radikalischen Polymerisation.
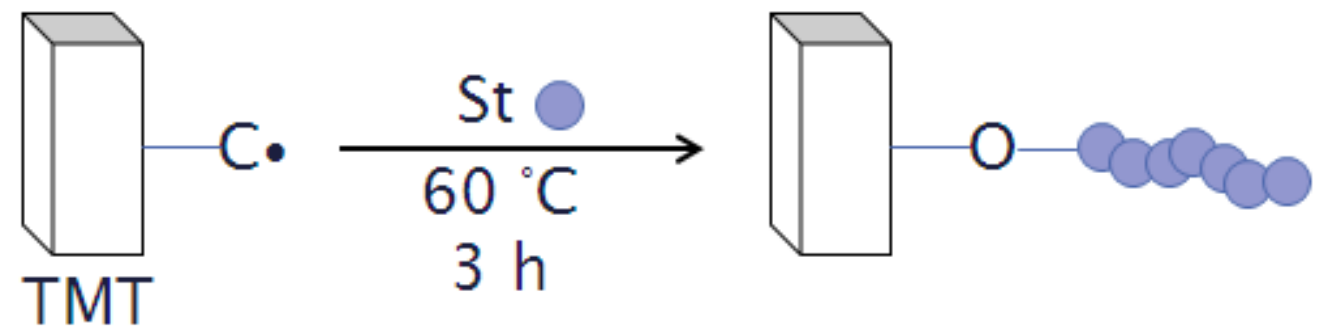

Schema 4.1: Schematische Darstellung der radikalischen Polymerisation des TMT Pulvers in der Substanz.

Vor den Experimenten wird TMT Pulver aus unterschiedlichen Modifikationsprozessen (s. oben) erst im Ofen bei $103^{\circ} \mathrm{C}$ ca. 24 Stunden 
getrocknet. Jede Suspension von $150 \mathrm{mg}$ TMT Pulver im 1,5 mL Styrol wird von Sauerstoff entgast. Anschließend wird die Polymerisation bei $60^{\circ} \mathrm{C}$ für 3 Stunden durchgeführt. Um die Polymerisationsreaktion abzubrechen, wird die Polymerisationsmischung mit Luft geflutet und im Eisbad abgekühlt. Anschließend wird TMT Pulver von den Polystyrol-Resten mit dem Toluol gewaschen und getrocknet.

\subsubsection{TGA Analyse}

Die thermogravimetrische Analyse (TGA) erschließt die Möglichkeit, temperaturabhängige Massenverluste von Polymeren quantitativ zu erfassen. ${ }^{99}$ Da die Zersetzungsphase für $\mathrm{Holz}$ und Polystyrol in unterschiedlichen Temperaturbereichen liegen und jeder Massenverlust als eine separate TG-Stufe gezeichnet wird, ist diese Methode gut geeignet, um das Polystyrol auf der Holzoberfläche zu bestimmen.

Auf der Abb. 4.1 oben sind die Thermogramme für das pulverisierte und mit St behandelte TM Buche mit unterschiedlichen Modifikationstemperaturen $\left(T_{\bmod } 180^{\circ} \mathrm{C}\right.$ (blau), $200{ }^{\circ} \mathrm{C}$ (orange), $220^{\circ} \mathrm{C}$ (rot)) mit den Thermogrammen von der thermisch unmodifizierten und mit St behandelten Buche (schwarz) und von der unmodifizierten und mit St nicht behandelten Buche als Referenz (schwarze Strichlinie) verglichen. Auf derselben Abbildung unten sind die ersten Ableitungen der Messsignale (dTG) präsentiert, um auch die kleinen oder schlecht aufgelösten TG-Stufen besser zu erkennen.

In der Tabelle 4.1 sind die Anfangszersetzungstemperaturen ( $\left.T_{\text {Anf }}\right)$ und die maximal schnellsten Abbautemperaturen $\left(T_{\max }\right)$ aus der TGA Analyse vorgestellt. Es ist deutlich zu erkennen, dass die mit St behandelten Proben mit den Modifikationstemperaturen $200{ }^{\circ} \mathrm{C}$ und $220^{\circ} \mathrm{C}$ zwei TG-Stufen enthalten. Die erste TG-Stufe liegt in dem Bereich zwischen ca. $225^{\circ} \mathrm{C}$ und $385^{\circ} \mathrm{C}$. Wie schon in der Abs. 2.1.1 bestimmt wurde, entsprechen diese Temperaturen den Zersetzungstemperaturen des Holzes. Die zweite TGStufe befindet sich zwischen $385^{\circ} \mathrm{C}$ und $556{ }^{\circ} \mathrm{C}$ und es passt zum Bereich des Massenverlustes für Polystyrol. ${ }^{100}$ 

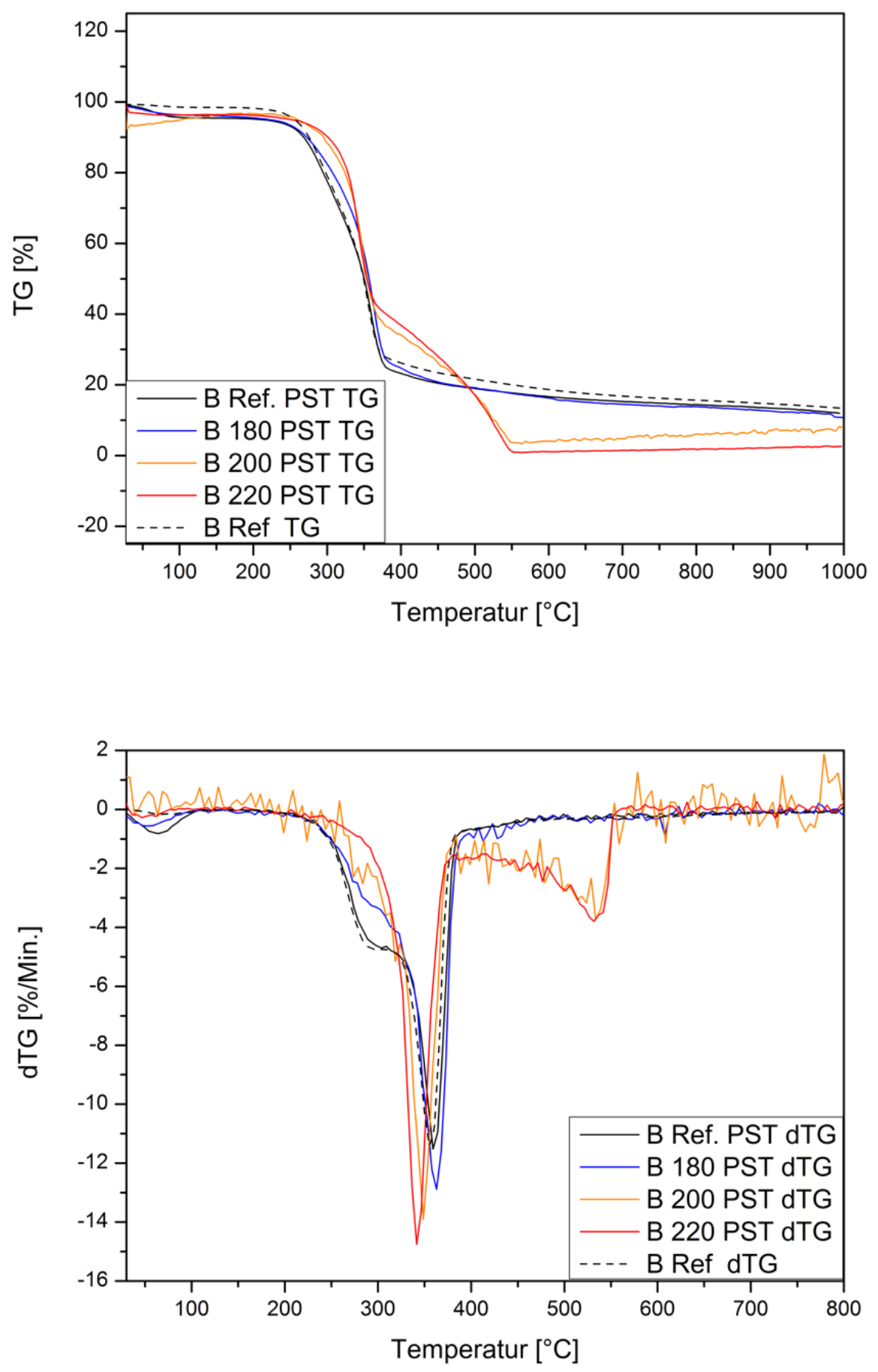

Abb. 4.1: Thermogramm (oben) und die erste Ableitung vom Thermogramm (unten) für Referenz Buche mit St (schwarz); für die thermisch modifizierte und mit St behandelte Buche mit folgenden Modifikationsparametern: $p_{\text {mod }} 5$ bar; $T_{\bmod } 180^{\circ} \mathrm{C}$ (blau), $200{ }^{\circ} \mathrm{C}$ (orange) sowie $220^{\circ} \mathrm{C}$ (rot) und für die Buche Referenz (Strichlinie). 
Tab. 4.1: Anfangszersetzungstemperaturen ( $\left.T_{\text {Anf }}\right)$ und maximal schnellste Abbautemperaturen $\left(T_{\max }\right)$ der unmodifizierten aber mit St behandelten Buche; der thermisch modifizierten und mit St behandelten Buche und der unmodifizierten und nicht behandelten Buche als Referenz.

\begin{tabular}{|c|c|c|c|c|}
\hline & \multicolumn{2}{|c|}{ 1. TG-Stufe } & \multicolumn{2}{c|}{ 2. TG-Stufe } \\
\hline Probe & $T_{\max }\left[{ }^{\circ} \mathrm{C}\right]$ & $T_{\text {Anf }}\left[{ }^{\circ} \mathrm{C}\right]$ & $T_{\max }\left[{ }^{\circ} \mathrm{C}\right]$ & $T_{\text {Anf }}\left[{ }^{\circ} \mathrm{C}\right]$ \\
\hline $\begin{array}{c}\text { Buche Ref. } \\
+ \text { PSt }\end{array}$ & 359 & 229 & - & - \\
\hline $\begin{array}{c}\text { Buche } 180{ }^{\circ} \mathrm{C} \\
+ \text { PSt }\end{array}$ & 362 & 231 & - & - \\
\hline $\begin{array}{c}\text { Buche } 200{ }^{\circ} \mathrm{C}+\text { PSt } \\
\text { Buche 220 }{ }^{\circ} \mathrm{C}+\text { PSt }\end{array}$ & 348 & 255 & 533 & 369 \\
\hline Buche Ref. & 355 & 227 & 531 & 367 \\
\hline
\end{tabular}

Die TGA Ergebnisse für die Kiefer sind auf der Abb. 4.2 vorgestellt. In der Abbildung oben sind die Werte des Massenverlustes für Referenz Kiefer mit St (schwarz); für die thermisch modifizierte und mit St behandelte Kiefer mit den Modifikationsparametern: $p_{\bmod } 5$ bar; $T_{\bmod } 180^{\circ} \mathrm{C}$ (blau), $200{ }^{\circ} \mathrm{C}$ (orange), $220^{\circ} \mathrm{C}$ (rot) und für die Kiefer Referenz (schwarze Strichlinie). Unten auf der Abbildung sind die ersten Ableitungen der Messsignale (dTG). Alle Kurven zeigen nur eine TG-Stufe in dem Temperaturbereich zwischen $235^{\circ} \mathrm{C}$ und $390^{\circ} \mathrm{C}$. Dieser Temperaturbereich entspricht nur dem Massenverlustbereich des Holzes (s. Abs. 2.1.1) und nicht des Polystyrols. In der Tabelle 4.2 sind die Anfangszersetzungstemperaturen ( $\left.T_{\text {Anf }}\right)$ und die maximal schnellsten Abbautemperaturen $\left(T_{\max }\right)$ aus der TGA Analyse für die Kiefer vorgestellt. 

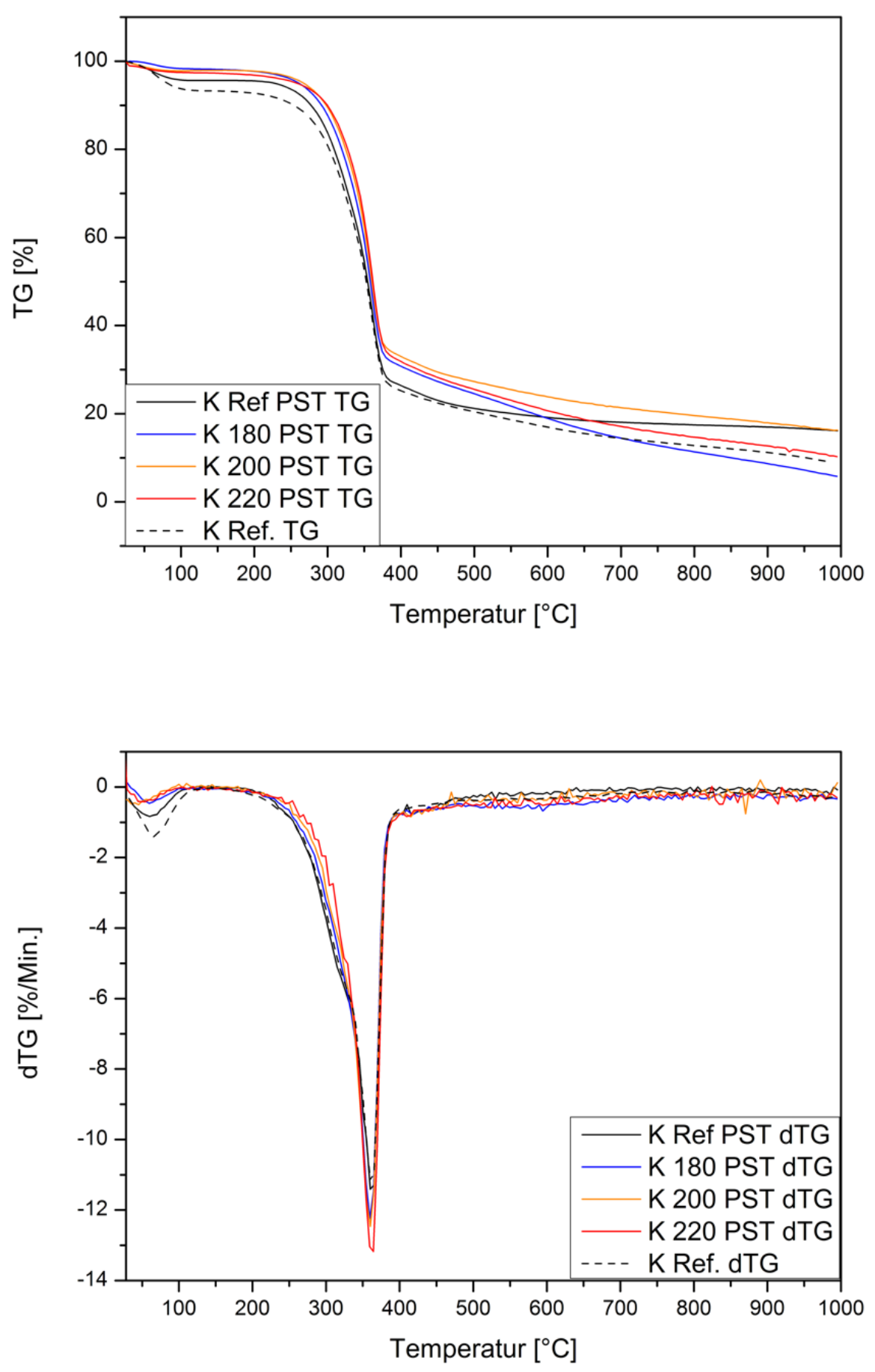

Abb. 4.2: Thermogramm (oben) und die erste Ableitung vom Thermogramm (unten) für Referenz Kiefer mit St (schwarz); für die thermisch modifizierte und mit St behandelte Kiefer mit folgenden Modifikationsparametern: $p_{\bmod } 5$ bar; $T_{\bmod } 180^{\circ} \mathrm{C}$ (blau), $200{ }^{\circ} \mathrm{C}$ (orange), $220^{\circ} \mathrm{C}$ (rot) und für die Kiefer Referenz (Strichlinie). 
Tab. 4.2: Anfangszersetzungstemperaturen ( $\left.T_{\text {Anf }}\right)$ und maximal schnellste Abbautemperaturen $\left(T_{\max }\right)$ der unmodifizierten aber mit St behandelten Kiefer; der thermisch modifizierten und mit St behandelten Kiefer und der unmodifizierten und nicht behandelten Kiefer als Referenz.

\begin{tabular}{|c|c|c|}
\hline Probe & $\boldsymbol{T}_{\max }\left[{ }^{\circ} \mathbf{C}\right]$ & $\boldsymbol{T}_{\text {Anf }}\left[{ }^{\circ} \mathbf{C}\right]$ \\
\hline Kiefer Ref.+ PSt & 360 & 238 \\
\hline Kiefer $180{ }^{\circ} \mathrm{C}+$ PSt & 359 & 239 \\
\hline Kiefer $200{ }^{\circ} \mathrm{C}+$ PSt & 360 & 239 \\
\hline Kiefer $220^{\circ} \mathrm{C}+$ PSt & 364 & 263 \\
\hline Kiefer Ref. & 359 & 235 \\
\hline
\end{tabular}

Infolgedessen bestätigen die TGA Ergebnisse, dass die Behandlung des TM Holzes mit Styrol durch die radikalische Polymerisation ohne Initiator möglich ist, aber nur unter bestimmten Bedingungen - die Konzentration der freien Radikalen in TMT sollte sehr hoch sein. Diesen Bedingungen entsprechen Proben aus der Buche mit Modifikationstemperaturen von $200{ }^{\circ} \mathrm{C}$ und $220^{\circ} \mathrm{C}$ (s. Abs. 2.2.2). Proben mit niedrigeren Radikalkonzentrationen sowohl für die Buche als auch für die Kiefer enthalten nur eine TG-Stufe und zeigen somit, dass Polystyrol auf der Oberfläche und in den Poren des Holzes nicht vorhanden ist oder, dass die Menge des Polystyrols so gering ist, dass sie durch TGA Analyse nicht zu bestimmen ist. Deswegen werden auch die anderen Analysenmethoden angewendet, um Polystyrol auf der Oberfläche und in den Poren des Holzes zu bestimmen.

\subsubsection{ATR-IR Analyse}

Die IR Analyse ist eine bessere Methode als TGA, um die detaillierten Veränderungen innerhalb der chemischen Struktur TMT zu erkennen. Auf der Abb. 4.3 sind die ATR-IR Spektren der thermisch modifizierten und mit Styrol behandelten Buche (oben) bzw. Kiefer (unten) mit folgenden Modifikationstemperaturen: $180^{\circ} \mathrm{C}$ (blau), $200^{\circ} \mathrm{C}$ (orange), $220^{\circ} \mathrm{C}$ (rot) und nicht modifizierten aber mit Styrol behandelten Proben (schwarz). 

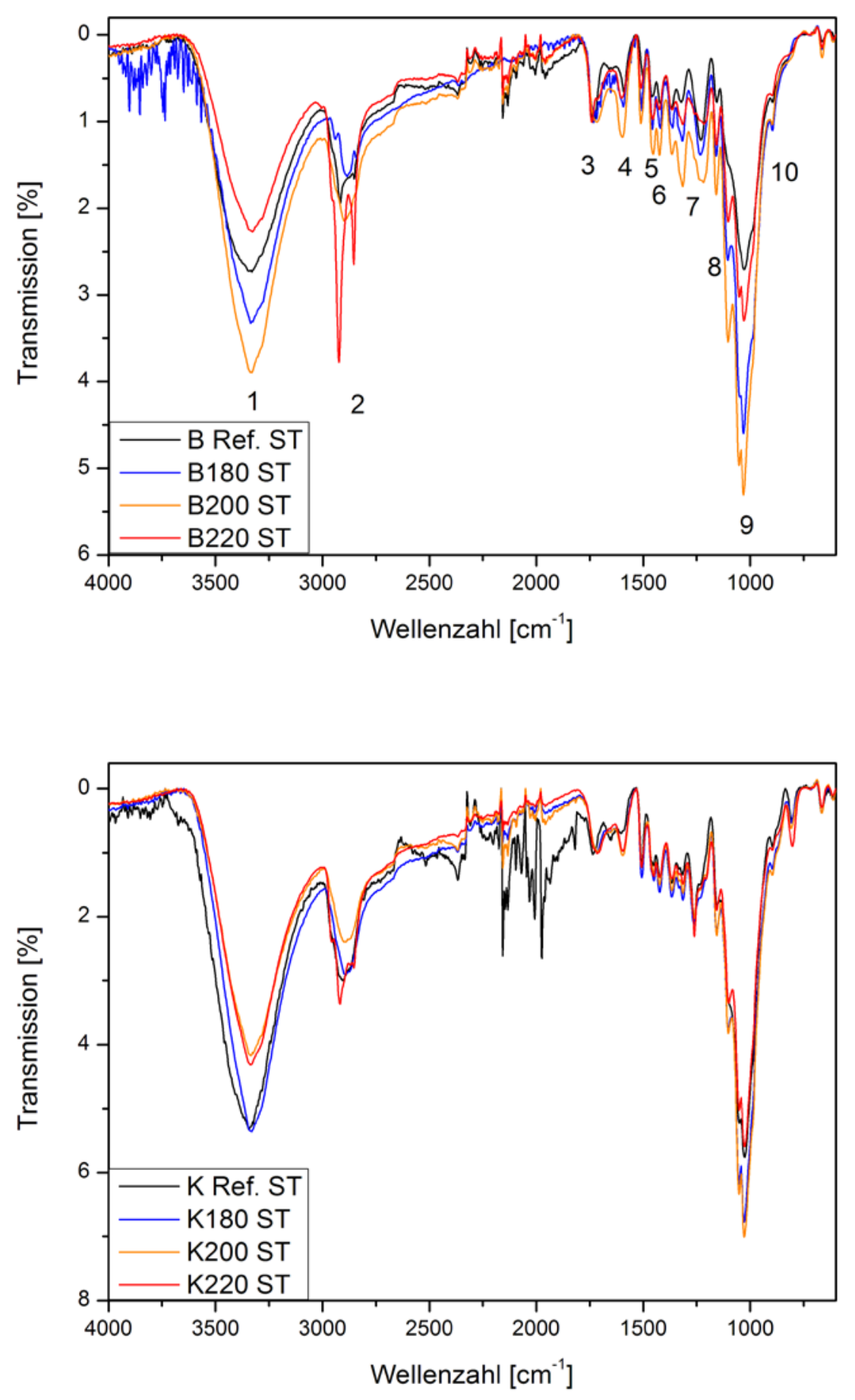

Abb. 4.3: ATR-IR Spektren für Buche (oben) bzw. Kiefer (unten), wobei thermisch nicht modifizierte und mit St polymerisierte Proben (schwarz) und thermisch modifizierte und mit St polymerisierte Proben mit Modifikationsdruck 5 bar; Modifikationstemperaturen $180{ }^{\circ} \mathrm{C}$ (blau), $200^{\circ} \mathrm{C}$ (orange) sowie $220^{\circ} \mathrm{C}$ (rot). 
Die Banden für ATR-IR Spektren der thermisch modifizierten und mit Styrol behandelten Buche bzw. Kiefer sind nach Esteves et al. zugeordnet und in der Tab. 4.3 vorgestellt. ${ }^{101}$

Tab. 4:3: Hauptbanden in ATR-IR Spektren des thermisch modifizierten und mit Styrol behandelten Holzes und ihre Zuordnung zu der Funktionalität. ${ }^{101}$

\begin{tabular}{|c|c|c|c|}
\hline Wellenlänge $\left[\mathrm{cm}^{-1}\right]$ & Nr. & Funktionalität & Schwingungen \\
\hline $3590-3100$ & 1 & $\begin{array}{l}\text { OH in Alkohol, } \\
\text { Phenol, Säure }\end{array}$ & Valenzschwingung \\
\hline $2990-2800$ & 2 & $\mathrm{CH}_{3}, \mathrm{CH}_{2}, \mathrm{CH}-$ & Valenzschwingung \\
\hline $1740-1727$ & 3 & $\begin{array}{c}\mathrm{C}=\mathrm{O} \text { in Ester } \\
\text { Ketone, } \\
\text { Aldehyde, }\end{array}$ & $\begin{array}{l}\text { nicht konjugierte } \\
\text { Valenzschwingung }\end{array}$ \\
\hline $1653-1504$ & 4 & $C=C$ & $\begin{array}{c}\text { konjugierte } \\
\text { Valenzschwingung }\end{array}$ \\
\hline $1455-1450$ & 5 & $\mathrm{CH}$ & Deformationsschwingung \\
\hline 1421 & 6 & $\begin{array}{l}\text { Arom. Ring } \\
\text { und } \mathrm{CH}\end{array}$ & Deformationsschwingung \\
\hline $1330-1230$ & 7 & $\begin{array}{l}\text { CO in S-, G- } \\
\text { Lignin }\end{array}$ & Valenzschwingung \\
\hline 1158-1106 & 8 & $\mathrm{CH}$ und $\mathrm{CO}$ & $\begin{array}{c}\mathrm{CH} \\
\text { Deformationsschwingung } \\
\text { in Lignin und } \\
\text { CO Valenzschwingung }\end{array}$ \\
\hline $1046-1023$ & 9 & C-O-C & Deformationsschwingung \\
\hline 840 & 10 & $\begin{array}{l}\mathrm{CH} \text { in } p- \\
\text { Position in } \\
\text { Aromaten }\end{array}$ & Deformationsschwingung \\
\hline
\end{tabular}

Die Normierung für diesen Fall ist nicht mehr möglich, weil die Intensität der Banden entweder während der thermischen Modifikation unter der Hitze oder während der Styrol Behandlung sich ändert. Aus diesem Grund werden die IR Spektren nicht quantitativ, sondern nur qualitativ verglichen.

In dem Wellenlängenbereich zwischen $2990 \mathrm{~cm}^{-1}$ und $2800 \mathrm{~cm}^{-1}$, in denen sich die $\mathrm{CH}_{3}, \mathrm{CH}_{2}, \mathrm{CH}$-Valenzschwingungen befinden, sind signifikante Unterschiede zwischen der modifizierten und mit St behandelten Buche 
mit $T_{\bmod } 220^{\circ} \mathrm{C}$ und den anderen Proben zu erkennen. In diesem Bereich liegen auch die asymmetrischen und symmetrischen $\mathrm{CH}$-Schwingungen von Polystyrol. ${ }^{102}$ Aber auch für die TM Buche mit $T_{\bmod } 200^{\circ} \mathrm{C}$ und für die TM Kiefer mit $T_{\text {mod }} 220^{\circ} \mathrm{C}$ sind die Polystyrol Banden in diesem Wellenlängenbereich zu erkennen.

Auf der Abb. 4.4 sind die IR Spektren vom Polystyrol (blau), von der TM Buche mit $T_{\bmod } 220^{\circ} \mathrm{C}$ (schwarz) und von der modifizierten $\left(T_{\bmod } 220^{\circ} \mathrm{C}\right)$ und mit Styrol behandelter Buche (rot) als Vergleich präsentiert.

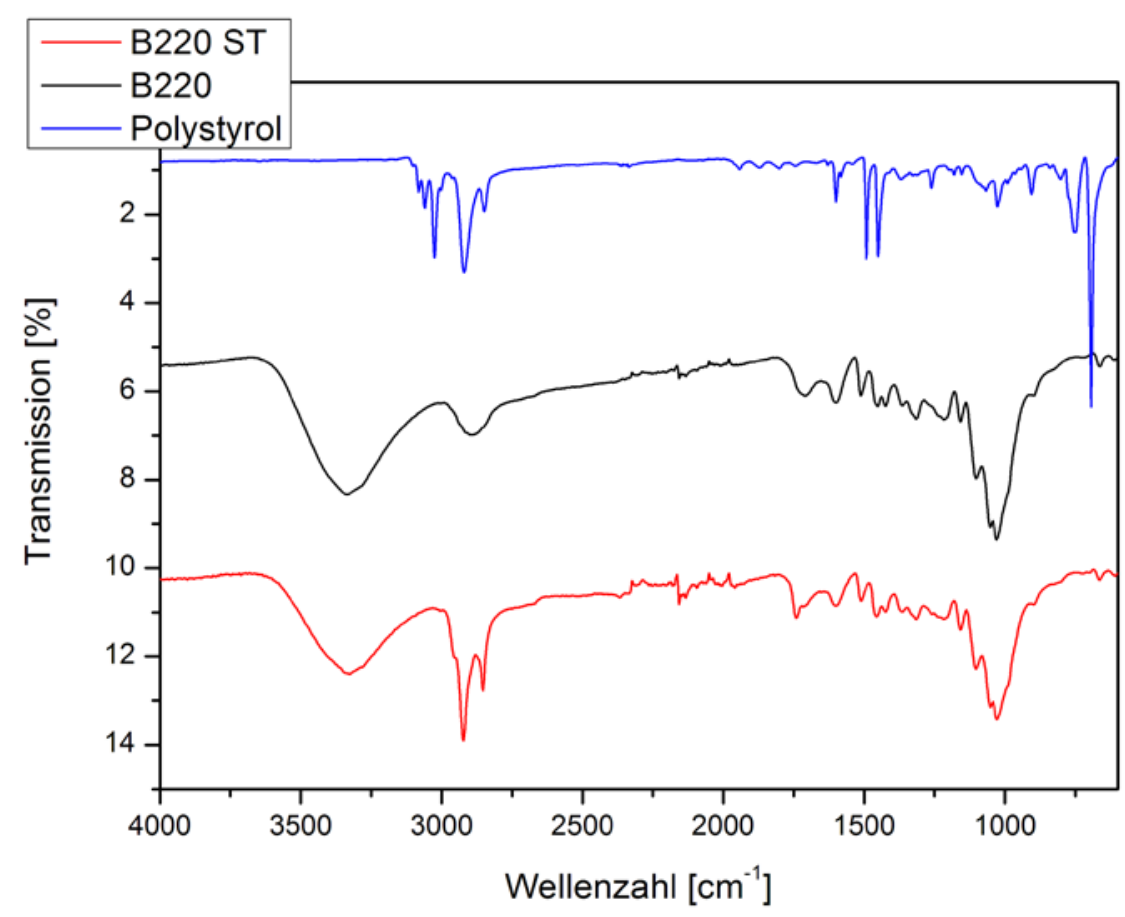

Abb. 4.4: Vergleich der ATR-FTIR Spektren von Polystyrol (blau) und thermisch modifizierter Buche $\left(T_{\bmod } 220^{\circ} \mathrm{C}, p_{\text {mod }} 5\right.$ bar) mit der thermisch modifizierten $\left(T_{\bmod } 220^{\circ} \mathrm{C}, p_{\text {mod }} 5\right.$ bar $)$ und weiter mit St polymerisierten Buche.

Infolgedessen bestätigen auch die ATR-IR Spektren, dass die freie Radikale in TMT für eine radikalische Polymerisationsreaktion verwendet werden können und die Reaktion ohne Initiator durchgeführt werden kann. Die Methode funktioniert sehr gut für die Proben mit der hohen Radikalkonzentration: Für die modifizierte Buche mit $T_{\bmod } 220^{\circ} \mathrm{C}$ oder $200{ }^{\circ} \mathrm{C}$ und auch für die modifizierte Kiefer mit $T_{\bmod } 220^{\circ} \mathrm{C}$. 


\subsubsection{Wasserkontaktwinkel (WCA)}

Die Änderungen der Hydrophobizität an der Oberfläche des behandelten Holzes werden mithilfe von Wasserkontaktwinkel (WCA, englisch Water Contact Angle) gemessen. Als erste wird das mit Styrol behandelte Holzpulver im Ofen bei $103{ }^{\circ} \mathrm{C}$ getrocknet und danach direkt in Tabs komprimiert, um eine flache Oberfläche zu erreichen.

Die Abb. 4.5 zeigt den Wasserkontaktwinkel (WCA) für das unmodifizierte und mit Polystyrol behandelte Holz (a-Kiefer; c-Buche) und für das thermisch modifizierte und mit Polystyrol behandelte Holz (b-Kiefer; dBuche) mit $T_{\bmod } 220^{\circ} \mathrm{C}$ und $p_{\text {mod }} 5$ bar.

a) WCA $93^{\circ}$

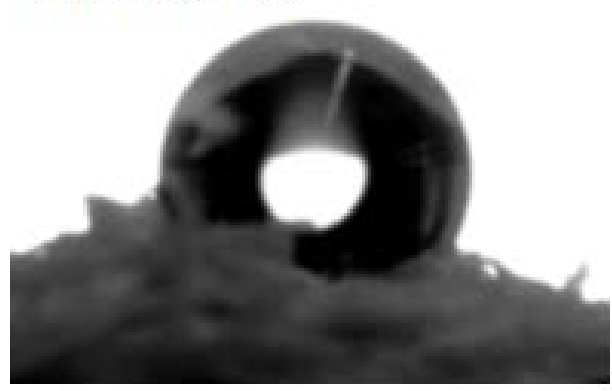

c) WCA $0^{\circ}$

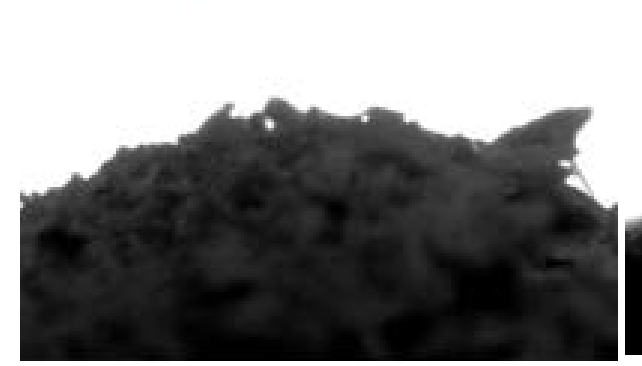

b) WCA $134^{\circ}$

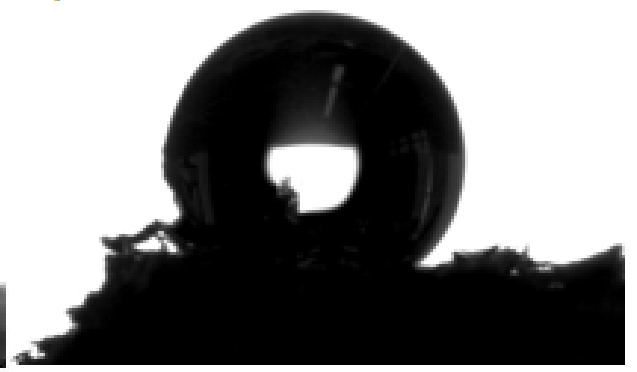

d) WCA $124^{\circ}$

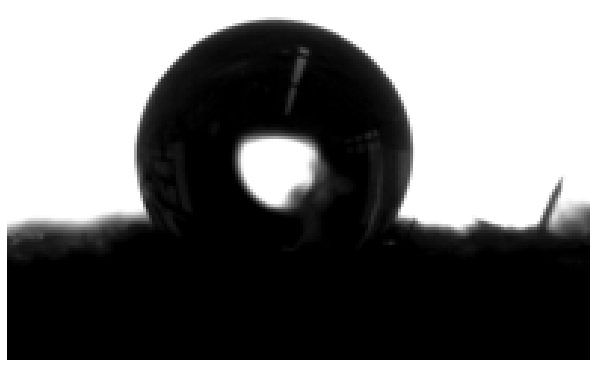

Abb. 4.5: Wasserkontaktwinkelmessungen: a) unmodifizierten Kiefer mit PSt $\left(93^{\circ}\right)$; b) mod. Kiefer mit PSt $\left(T_{\bmod } 220^{\circ} \mathrm{C}, p_{\text {mod }} 5\right.$ bar $)\left(134^{\circ}\right)$; c) unmodifizierten Buche mit PSt $\left(0^{\circ}\right)$; d) mod. Buche mit PSt $\left(T_{\bmod } 220^{\circ} \mathrm{C}, p_{\bmod } 5\right.$ bar $)\left(124^{\circ}\right)$.

Obwohl das Polystyrol hydrophob ist, ${ }^{103}$ ist die unmodifizierte mit St behandelte Buche sehr hydrophil aufgrund zahlreicher Hydroxy-Gruppen und kleinerer Mengen vom Polymer an der Oberfläche. Deswegen wird das Wassertröpfchen von der Oberfläche schnell absorbiert, was zur Quellung der gepressten Probe führt (s. Abb. 4.5, c). Interessanterweise ist die unmodifizierte aber mit dem Polystyrol behandelte Kiefer ziemlich hydrophob und das Wassertröpchfen bleibt an der Oberfläche über längere 
Zeit mit WCA $93^{\circ}$ (s. Abb. 4.5, a). Die Unterschiede der Hydrophobizität zwischen der Buche und der Kiefer sind dadurch erklärt, dass der Hemicellulose-Anteil im Nadelholz kleiner als im Laubholz ist ${ }^{1}$ und die $\mathrm{OH}-$ Gruppen in der Hemicellulose um den Faktor 1,6 hydrophyler als in der Cellulose sind. Daher nehmen sie sehr viel Wasser auf. ${ }^{104}$

Die WCA Messungen zeigen, dass die thermisch modifizierte und mit PSt behandelte Kiefer insgesamt hydrophober ist als einfach thermisch modifizierte Kiefer. Der Wasserkontaktwinkel liegt zwischen $108^{\circ}$ und $134^{\circ}$ für die Holzproben mit $T_{\bmod } 180^{\circ} \mathrm{C}, 200^{\circ} \mathrm{C}, 220^{\circ} \mathrm{C}$. Für die Buche ist die Hydrophobizität niedriger als für einfach thermisch modifizierte Buche. Nur die Buche mit höherer Modifikationsstufe $T_{\bmod } 220^{\circ} \mathrm{C}$ zeigt verbesserte Werte des WCA von $124^{\circ}$.

Es ist schwierig stabile Tabs für die WCA Messungen aus dem Holzpulver zu komprimieren, weil das Holzpulver sehr trocken ist, die Holzpartikel ziemlich groß sind - die Größe der Partikel liegt zwischen 0,2 und 0,63 mm - die Hohlräume zwischen den Teilchen sind deswegen auch sehr groß. Die Instabilität der Holzproben hat die Messwerte der Hydrophobizität verschlechtert. Deswegen wird eine andere Methode - dynamische Wasserdampfsorption (DVS) - verwendet, um die Hydrophobizität zu untersuchen.

\subsubsection{Dynamische Wasserdampfsorption (DVS)}

Mit Hilfe vom DVS-Gerät (englisch Dynamic Vapor Sorption) werden die Sorption-Isothermen aufgenommen. DVS ist eine gravimetrische Methode, um den Gleichgewichtsfeuchtegehalt (EMC englisch Equilibrium Moisture Content) in hydrophilen Materialien zu bestimmen. ${ }^{105}$ Dafür wird der Prüfkörper mit der Masse von 20 mg in einer abgeschlossenen Kammer bei einer konstanten Raumtemperatur von $25^{\circ} \mathrm{C}$ gravimetrisch gemessen. Die relative Luftfeuchtigkeit ( $\mathrm{RH}$ englisch Relative Humidity) wird schrittweise zuerst von $0 \%$ auf $90 \%$ mit einer Rate von $10 \%$ und anschließend auf 95\% erhöht und danach wieder auf $0 \%$ in der umgekehrten Reihenfolge abgesenkt. Wenn die gewünschte relative Luftfeuchtigkeit erreicht wird, bleibt sie konstant solange die Gewichtsänderung der Probe pro Minute weniger als 0,002\% $\mathrm{min}^{-1}$ über einen Zeitraum von 10 Minuten ist. Die Änderungen der relativen Luftfeuchtigkeit und der Massenänderung des gravimetrischen Gleichgewichts werden als Funktion der Zeit aufgetragen.106 Die Adsorptions- und Desorptionseigenschaften vom thermisch modifizierten 
und mit St behandelten Holz werden entweder als Gleichgewichtsfeuchtigkeit (EMC) oder als reduzierter Gleichgewichtsfeuchtigkeitsgehalt $\left(\mathrm{EMC}_{\mathrm{R}}\right)$ eingegeben.

EMC und $\mathrm{EMC}_{\mathrm{R}}$ werden mit der Gl. 4.1 und der Gl. 4.2 berechnet, ${ }^{107}$

$$
\begin{gathered}
\mathrm{EMC}=\frac{\left(m_{2}-m_{1}\right)}{m_{1}} \cdot 100 \% \\
\mathrm{EMC}_{\mathrm{R}}=\mathrm{EMC} \cdot\left(1+\frac{\mathrm{WPG}}{100}\right)
\end{gathered}
$$

wobei $m_{2}$ das Gewicht der Probe im Gleichgewicht bei einer gegebenen relativen Luftfeuchtigkeit ist, $m_{1}$ das darrtrockene Gewicht der Probe ist und WPG die prozentuale Gewichtszunahme der Probe aufgrund ihrer Behandlung mit St ist.

WPG wird mit der Gl. 4.3 berechnet,

$$
\mathrm{WPG}=\frac{\left(m_{(\mathrm{Holz}+\text { Polymer })}-m_{(\mathrm{Holz})}\right)}{m_{(\mathrm{Holz})}} \cdot 100 \%
$$

wobei $m_{\text {(Holz+Polymer) }}$ das Gewicht der darrgertockneten modifizierten Holzprobe mit PSt ist und $m_{(\mathrm{Holz})}$ das Gewicht der darrgertrockneten Holzprobe vor der Behandlung mit PSt. ${ }^{108}$

Auf der Abb.4.6 ist eine Auftragung EMC gegen $\mathrm{RH}$ in \% mit der Adsorption- und Desorptionsisothermen der thermisch modifizierten Buche $\left(T_{\text {mod }} 220^{\circ} \mathrm{C}, p_{\text {mod }} 5 \mathrm{bar}\right.$; rot), der thermisch modifizierten $\left(T_{\bmod } 220^{\circ} \mathrm{C}, p_{\text {mod }} 5\right.$ bar) und weiter mit St behandelten Buche (blau) und nicht modifizierter Buche als Referenz (schwarz) dargestellt. Es ist deutlich $\mathrm{zu}$ erkennen, dass die Adsorptions- und Desorptionsisothermen unterschiedlich verlaufen, was zu einer Hysterese führt. Die Isothermen des nicht modifizierten Holzes (schwarz) haben eine typische Sigmoidform für das Laubholz. ${ }^{109}$ Die thermische Modifikation des Holzes führt zu einer starken Abnahme der Adsorptions- und Desorptionskurven und der EMC Wert übersteigt 10\% nicht (rot). Die Behandlung des TMT mit Styrol sollte zum größeren hydrophoben Effekt führen, aber die DVS Ergebnisse zeigen keinen signifikanten Unterschied zwischen TMT (rot) und behandeltes mit Styrol TMT (blau). 


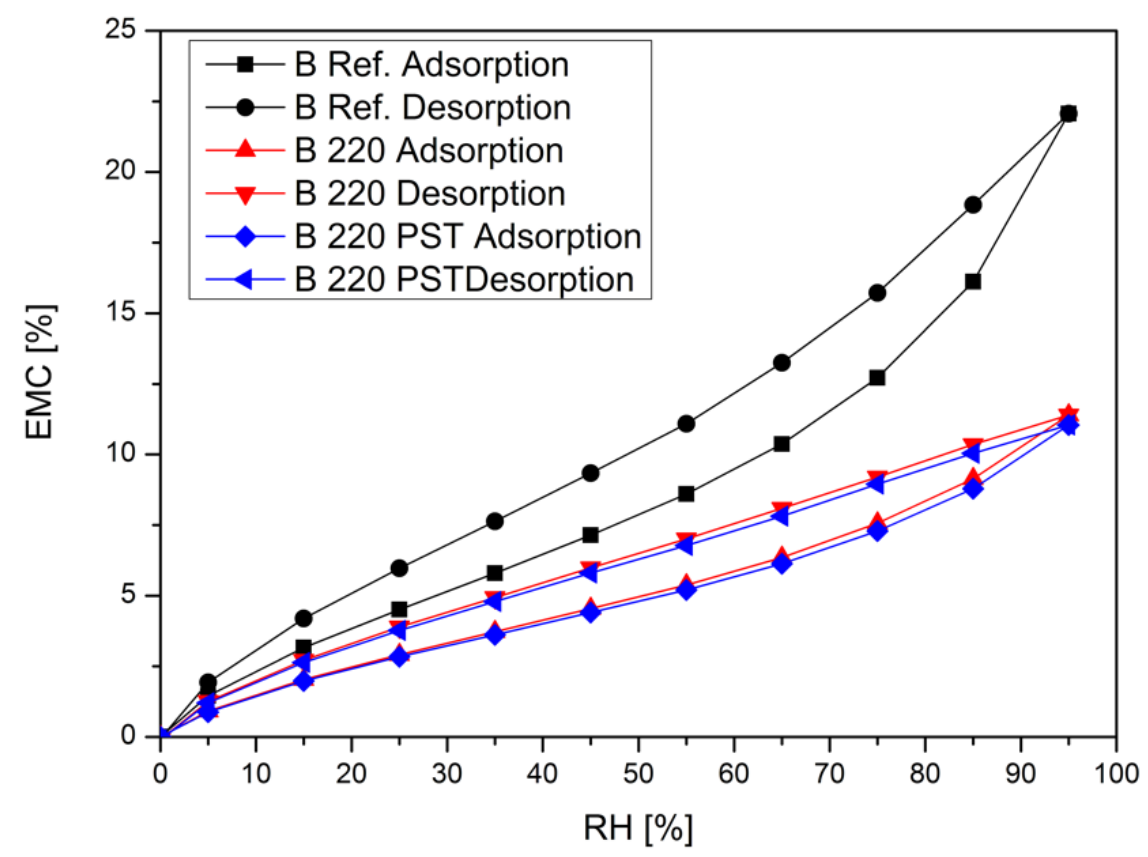

Abb. 4.6: Auftragung EMC gegen $\mathrm{RH}$ in \%. Adsorption und Desorption Isothermen der thermisch modifizierten Buche $\left(T \bmod 220^{\circ} \mathrm{C}, p_{\bmod } 5 \mathrm{bar}\right)$ (rot), der thermisch modifizierten $\left(T \bmod 220^{\circ} \mathrm{C}, p_{\text {mod }} 5 \mathrm{bar}\right)$ und weiter mit St polymerisierten Buche (blau) und nicht modifizierter Buche als Referenz (schwarz).

Es liegt daran, dass die Styrolmoleküle sehr groß und hydrophob sind und nicht in der Zellwand des Holzes reingehen, sondern bleiben nur an der Oberfläche.

Dieser Effekt ist deutlicher auf der nächsten Abbildung zu erkennen. Hier ist eine Auftragung $\mathrm{EMC}_{\mathrm{R}}$ gegen $\mathrm{RH}$ in \% dargestellt. Im Gegensatz zu der Abb. 4.6 sind die DVS Ergebnisse auf der Abb. 4.7 nur für das Holz und nicht für das gesamte Material (Holz mit Polystyrol). Das thermisch modifizierte Holz selbst nach der Behandlung mit Styrol ist nicht hydrophober geworden. Im Gegenteil die Hydrophobizität des Holzes hat sich verschlechtert und $\mathrm{EMC}_{\mathrm{R}}$ ist fast 13\% (blau). Die Werte des nicht modifizierten Holzes (schwarz) und des thermisch modifizierten Holzes (rot) bleiben konstant, weil es hier kein Polymer gibt. Das beweist, dass Polystyrol sich nur an der Oberfläche des Holzes befindet und nicht in der Zellwand drin. Deswegen wird in dieser Arbeit die Wortverbindung "eine chemische Modifikation mit Styrol" vermieden und nur das Wort „Behandlung“ benutzt. 


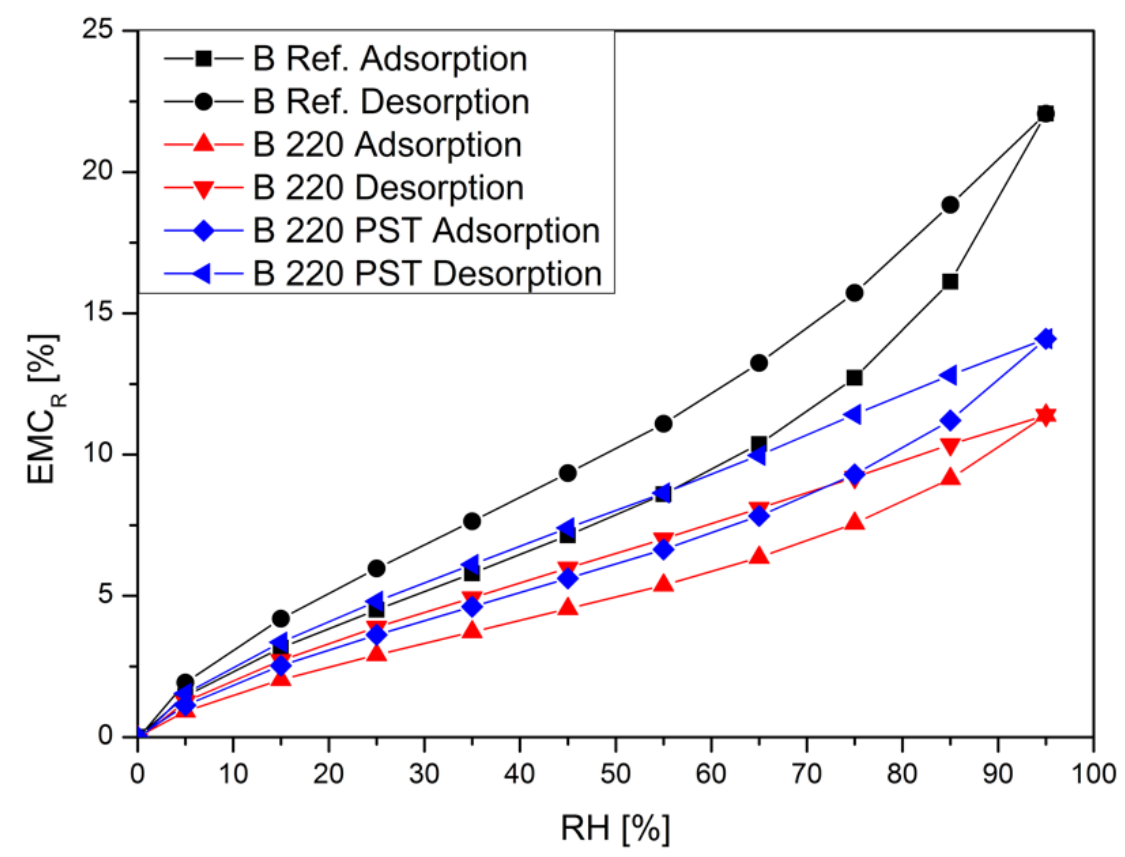

Abb. 4.7: Auftragung $\mathrm{EMC}_{\mathrm{R}}$ gegen $\mathrm{RH}$ in \%. Adsorption und Desorption Isothermen der thermisch modifizierten Buche $\left(T_{\bmod } 220^{\circ} \mathrm{C}, p_{\bmod } 5 \mathrm{bar}\right)(\mathrm{rot})$, der thermisch modifizierten $\left(T_{\bmod } 220^{\circ} \mathrm{C}, p_{\bmod } 5 \mathrm{bar}\right)$ und weiter mit St polymerisierten Buche (blau) und nicht modifizierter Buche als Referenz (schwarz).

\subsection{Radikalische Polymerisation des TMT Pulvers in der Lösung ohne Initiator}

Hier werden zwei unterschiedliche Monomere verwendet: zuerst Styrol, aufgrund seiner guten Polymerisationseigenschaften, danach Na-4-Vinylbenzolsufonat (NaStS) wegen der hydrophilen Eigenschaft. Als Lösungsmittel wird DMSO ausgewählt, weil diese Lösung das Holz besser lösen kann als andere Lösungen.

Für die Blindversuche mit St wird TMT Pulver von der thermisch modifizierten Kiefer und Buche mit den Modifikationsparametern $T_{\text {mod }} 180^{\circ} \mathrm{C}, 200^{\circ} \mathrm{C}$ und $220^{\circ} \mathrm{C}$ und mit $p_{\text {mod }} 5$ bar im Ofen 24 Stunden bei $103^{\circ} \mathrm{C}$ getrocknet. Danach werden je $110 \mathrm{mg}$ TMT Pulver in $2 \mathrm{~mL}$ DMSO über Nacht gelöst und anschließend mit je 1,1 mL St gerührt. Alle Proben werden mit dem Argon von Sauerstoff 20 Minuten lang entgast. Die Polymerisationsreaktion wird bei $60^{\circ} \mathrm{C} 20$ Stunden durchgeführt. Zum Polymerisationsabbruch werden die Mischungen mit Luft geflutet und im Eisbad abgekühlt. Zum Schluss wird das behandelte TMT Pulver mit dem 
Toluol jeweils dreimal gewaschen und getrocknet.

Als Analysemethoden werden TGA und IR-Spektroskopie verwendet.

Abb. 4.8 zeigt die Thermogramme (oben) und ihre ersten Ableitungen (unten) für die Referenz Buche, die mit St behandelt wurde (schwarz) und die thermisch modifizierte $\left(T_{\bmod } 220^{\circ} \mathrm{C}\right)$ und mit $S t$ behandelte Buche (rot).

Die TGA Ergebnisse zeigen, dass alle Thermogramme nur eine TG-Stufe enthalten. Diese TG-Stufe liegt in dem Temperaturbereich zwischen ca. $265^{\circ} \mathrm{C}$ und $360^{\circ} \mathrm{C}$, was einem Bereich für den Holzabbau entspricht. ${ }^{110}$ Nur die Probe, die bei $220^{\circ} \mathrm{C}$ modifiziert wurde und erst danach mit dem St in der Lösung behandelt wurde, hat zwei TG-Stufen. Erste TG-Stufe liegt wie erwartet in dem Bereich für den Holzabbau und die zweite TG-Stufe liegt zwischen $360^{\circ} \mathrm{C}$ und $655^{\circ} \mathrm{C}$. Dieser Bereich entspricht den TGAWerten für Polystyrol. ${ }^{98}$ Die zweite TG-Stufe ist sehr klein und schlecht aufgelöst, aber sie wird deutlicher auf der Ableitung des Thermogramms (unten, rot). Daraus lässt sich schließen, dass die radikalische Polymerisation des TMT mit Styrol in der Lösung ohne Initiator nicht erfolgreich war. Die freien stabilen Radikale aus TMT reagieren ab, wenn das thermisch modifizierte Holz in DMSO gelöst wird. Deswegen können sie in der radikalischen Polymerisationsreaktion ohne Initiator nicht teilnehmen. Nur die Probe mit einer hohen Radikalkonzentration wie Buche mit $T_{\bmod } 220^{\circ} \mathrm{C}$ zeigt die Verfügbarkeit einer kleinen Menge des Polystyrols an der Oberfläche des TMT. Es liegt daran, dass stark modifiziertes Holz sehr schwer zum Lösen ist. Deswegen bleiben einige freie Radikale in den ungelösten Holzpartikeln übrig und reagieren mit Styrol ab.

Leider bestätigen auch die IR Ergebnisse, dass die radikalische Polymerisation des TMT Pulvers mit Styrol in der Lösung ohne Initiator nicht funktioniert hat, und zeigen auf den IR-Spektren keine signifikanten Unterschiede (s. Anhang). 

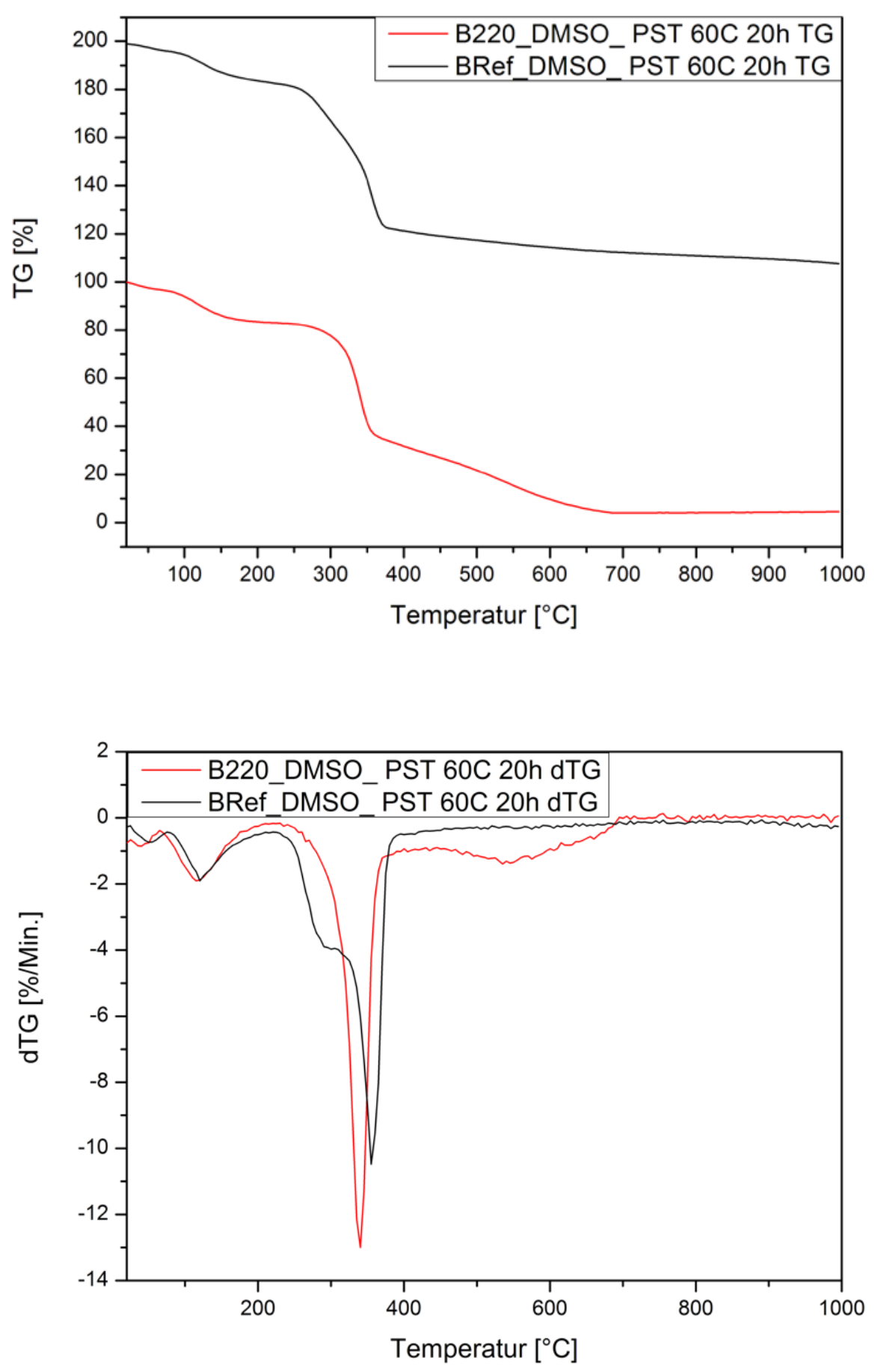

Abb. 4.8: Thermogramm (oben) und die erste Ableitung vom Thermogramm (unten) für nicht modifizierte und mit St behandelte Buche(schwarz); und für die thermisch modifizierte und mit St behandelte Buche $\left(T_{\bmod } 220^{\circ} \mathrm{C}\right.$, rot). Die Polymerisationsreaktion wurde in DMSO bei $60^{\circ} \mathrm{C}$, 20 Stunden durchgeführt. 
Styrol ist ein hydrophobes Molekül, deswegen kann St nur an der Oberfläche des Holzes während der radikalischen Polymerisation angreifen und nicht in der Zellwand des Holzes rein gehen. Aus diesem Grund wird ein hydrophileres Monomer NaStS weiter getestet, damit Monomer direkt in der Zellwand des Holzes polymerisieren kann und nicht nur an der Oberfläche. Da NaStS ein Feststoff ist, muss die Polymerisationsreaktion auch in der Lösung gemacht werden. Dafür wird auch TMT Pulver von der thermisch modifizierten Kiefer und Buche verwendet, mit den folgenden TMT-Parametern: $T_{\bmod } 180^{\circ} \mathrm{C}, 200{ }^{\circ} \mathrm{C}$ und $220^{\circ} \mathrm{C}$; und $p_{\bmod } 5$ bar. Das TMT Pulver wird zuerst im Ofen bei $103^{\circ} \mathrm{C}$ 24 Stunden getrocknet. Danach werden $110 \mathrm{mg}$ TMT Pulver in $2 \mathrm{~mL}$ DMSO gelöst und mit $100 \mathrm{mg}$ NaStS gerührt. Zur Sauerstoffbefreiung der Proben wird die Spülung mit Argon jeweils 20 Minuten lang verwendet. Die Polymerisationsreaktion wird bei $75^{\circ} \mathrm{C} 20$ Stunden durchgeführt. Um die Polymerisationsreaktion abzubrechen, wird die Polymerisationsmischung mit Luft geflutet und im Eisbad abgekühlt. Anschließend wird das behandelte mit NaStS TMT Pulver mehrmals mit Wasser ausgewaschen, getrocknet und analysiert.

Abb. 4.9 zeigt die IR Spektren für die TM Buche (oben) und die TM Kiefer (unten), die mit NaStS behandelt wurden. Hier sind keine IR-Banden von $\mathrm{NaStS}$ zu erkennen. Alle Unterschiede, die hier vorkommen, sind nur von der thermischen Modifikation und von den inneren Strukturänderungen durch die Hitze begründet.

Diese beiden Blindversuche mit unterschiedlichen Monomeren zeigen, dass die radikalische Polymerisation des TMT mit St oder mit NaStS in der Lösung ohne den Initiator nicht möglich ist. Die freien Radikale von TMT in einer gelösten Form in der Lösung reagieren miteinander ab und können in der Reaktion nicht mehr teilnehmen. Aus diesem Grund werden keine weiteren Analysen und Versuche in der Lösung durchgeführt. 

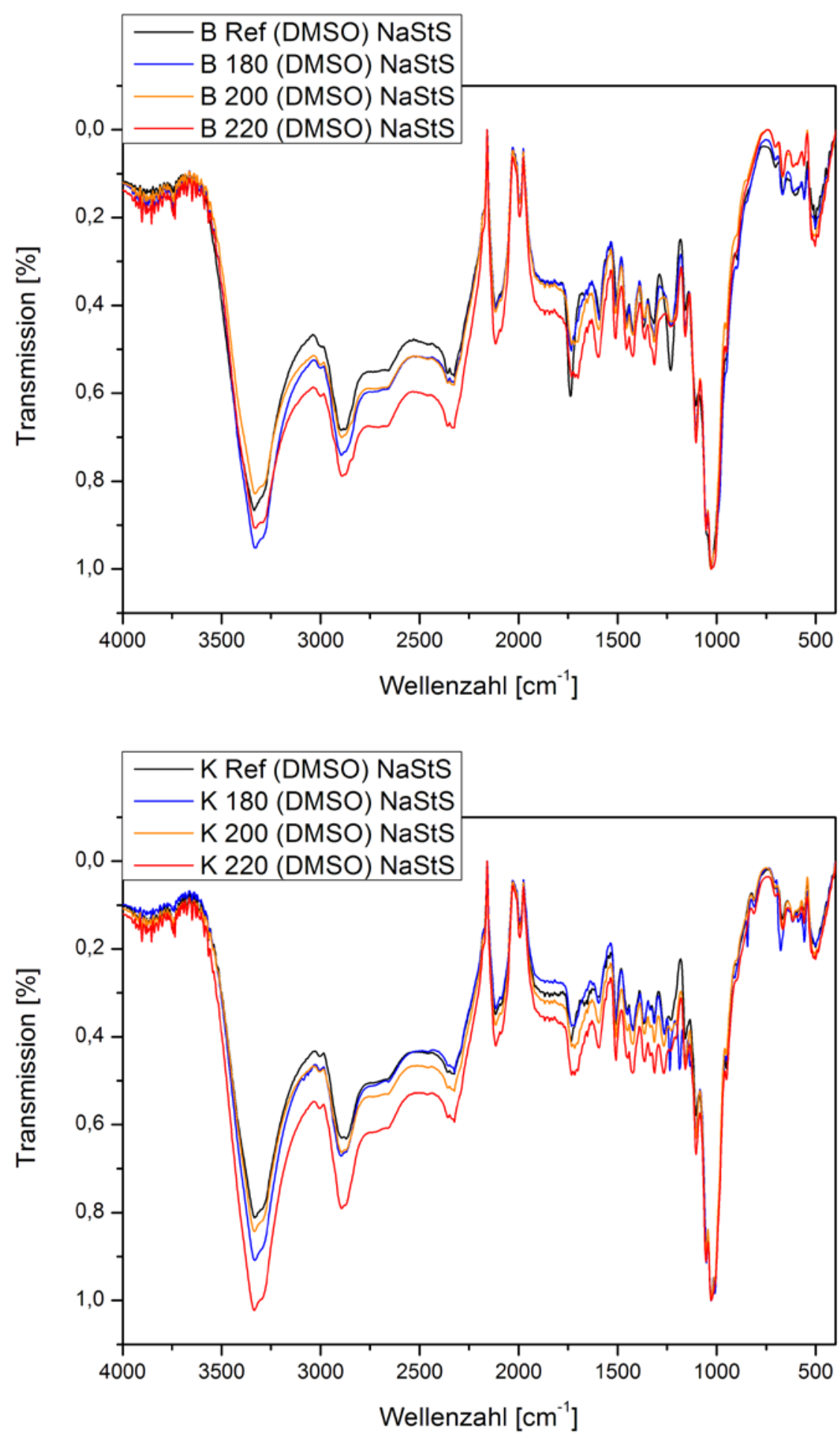

Abb. 4.9: ATR-IR Spektren für Buche (oben) bzw. Kiefer (unten), wobei thermisch nicht modifizierte und mit NaStS polymerisierte Probe (schwarz) und thermisch modifizierte und mit NaStS polymerisierte Proben mit Modifikationsdruck 5 bar; Modifikationstemperaturen $180^{\circ} \mathrm{C}$ (blau), $200^{\circ} \mathrm{C}$ (orange) sowie $220^{\circ} \mathrm{C}$ (rot). 


\subsection{ESR Messungen während der Polymerisationsreaktion}

Um zu bestätigen, dass die stabilen freien Radikale aus TMT tatsächlich in der Polymerisationsreaktion in der Substanz teilnehmen, werden die ESR Messungen direkt während der Reaktion drei Stunden ständig durchgeführt.

Für den Versuch wird nur thermisch modifizierte pulverisierte Buche mit $T_{\text {mod }} 220^{\circ} \mathrm{C}$ und $p 5$ bar verwendet, weil IR- und TGA-Analysen für diese Probe sehr guten Ergebnissen gezeigt haben. Als Erstes wird das Holzpulver bei $103{ }^{\circ} \mathrm{C}$ getrocknet und danach mit Argon 2 Stunden entgast. Styrol wird durch drei freeze-pump-and-thaw-Zyklen von Sauerstoff entgast. Das entgaste Monomer sowie das entgaste TMT Pulver werden in eine Glovebox mit der Argon-Atmosphäre geschleust. Dort wird eine Probe aus $15 \mathrm{mg}$ TMT Pulver und 0,2 mL St direkt in den ESR-Röhrchen vorbereitet und luftdicht verschlossen.

Während der permanenten ESR-Messungen wird eine Polymerisationsreaktion bei $60^{\circ} \mathrm{C} 3 \mathrm{~h}$ direkt in einem ESR-Röhrchen durchgeführt. Alle 10 Minuten wird drei Mal die Radikalkonzentration gemessen. Nach drei Stunden wird die Reaktion abgebrochen.

Abb. 4.10 zeigt eine Auftragung der Werte des Integrals des Absorptionssignals gegen Zeit der radikalischen Polymerisation für TMT mit dem Styrol. Es ist ganz deutlich $\mathrm{zu}$ erkennen, dass die Radikalkonzentration in der ersten Stunde sinkt und in den nächsten zwei Stunden relativ konstant bleibt. 


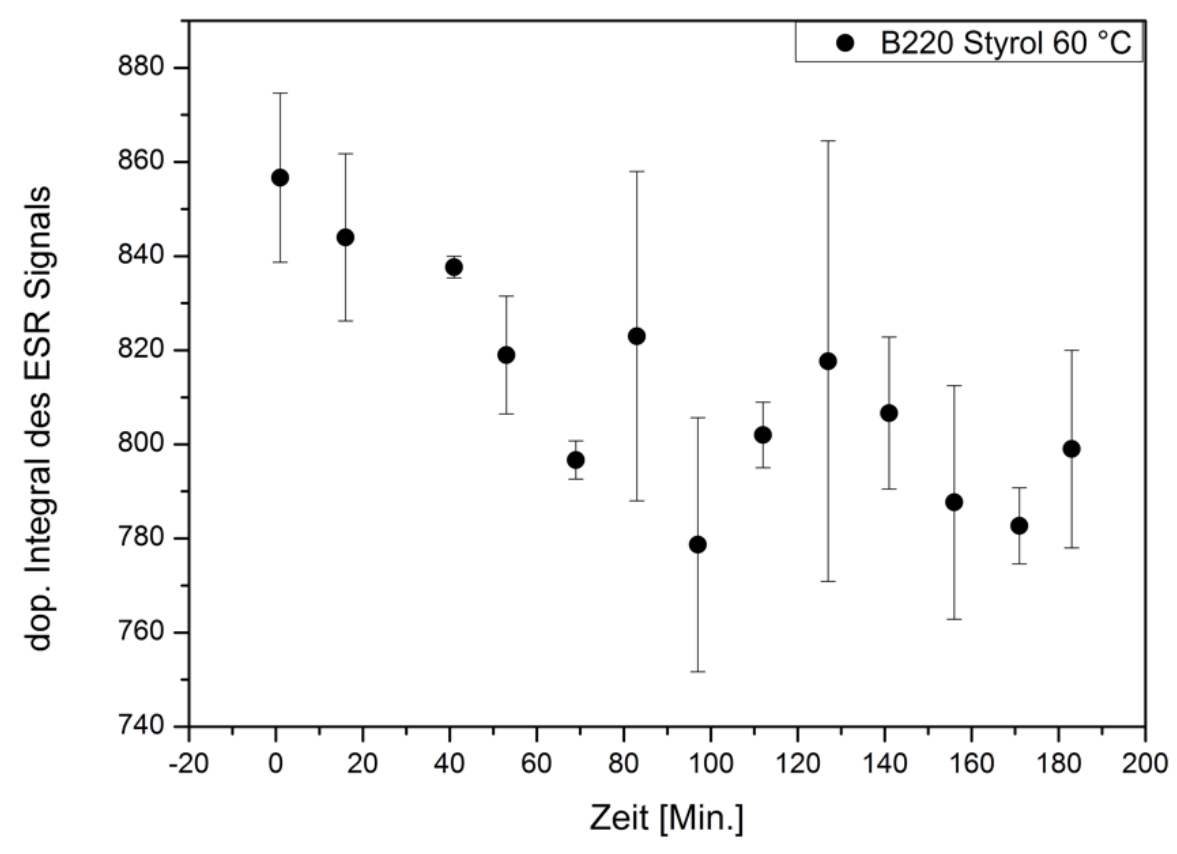

Abb. 4.10: Auftragung des Integrals des Absorptionssignals gegen Zeit der radikalischen Polymerisation des TMT Pulvers $\left(T_{\bmod } 220^{\circ} \mathrm{C}, p_{\bmod } 5\right.$ bar $)$ mit St in Minuten. Die ESR Messung wurde bei $60^{\circ} \mathrm{C}$ drei Stunden lang durchgeführt.

Anschließend werden die Blindversuche nur mit TMT bei $60^{\circ} \mathrm{C}$ (s. Abb. 4.11, rot) und mit TMT in dem Toluol bei $60^{\circ} \mathrm{C}$ (s. Abb. 4.11, schwarz) über längere Zeit durchgeführt. Die Ergebnisse sind in der Abb.4.11 dargestellt.

In diesem Fall bleibt die Radikalkonzentration in den Blindproben über längere Zeit relativ konstant. Nur nach den ersten 10 Minuten steigt die Radikalkonzentration in der Probe, die nur aus TMT besteht (rot), aufgrund von der Temperaturerhöhung auf $60^{\circ} \mathrm{C}$. Diese Blindversuche zeigen, dass die Radikalkonzentration niedriger wird (s. Abb.4.10), weil den freien stabilen Radikalen in der Polystyrolpolymerisationsreaktion teilnehmen und nicht, weil sie miteinander abreagieren. 


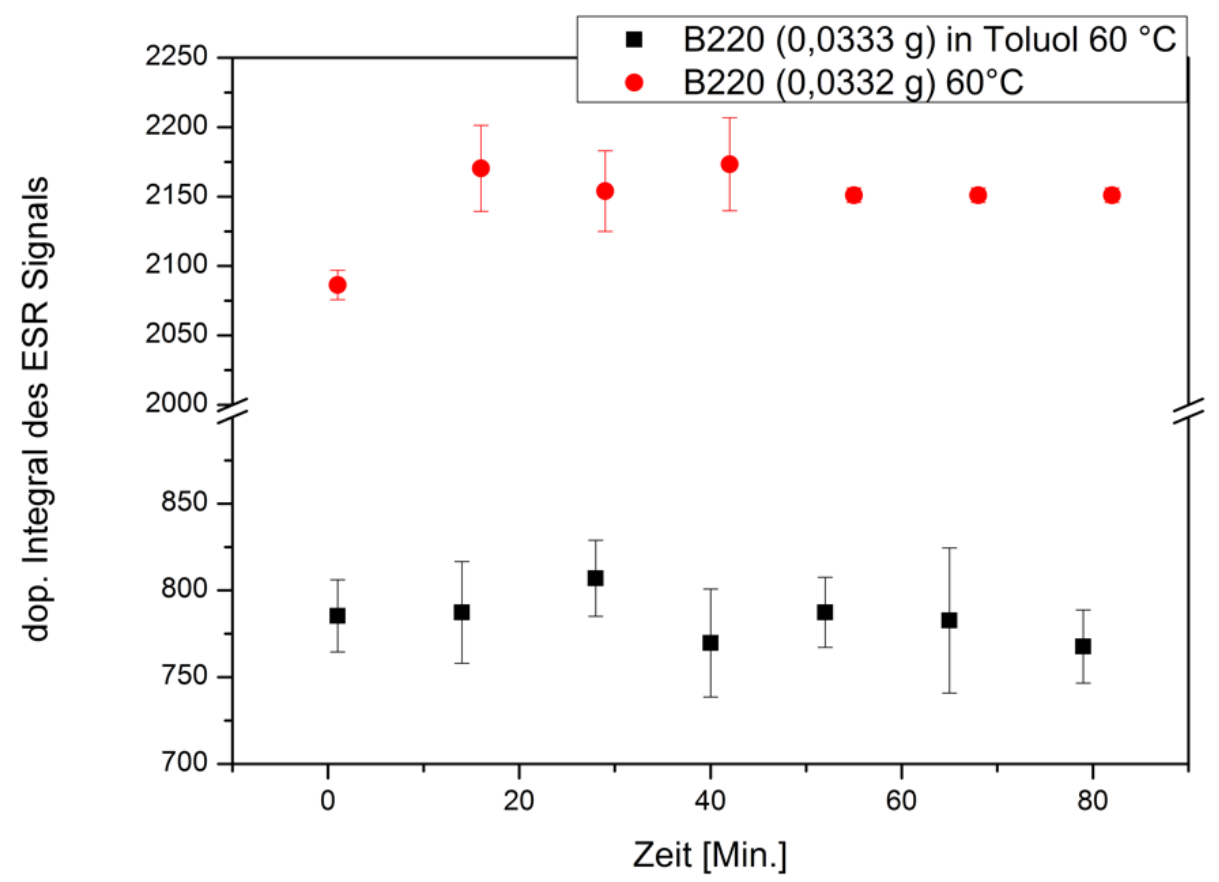

Abb. 4.11: Auftragung des Integrals des Absorptionssignals gegen Zeit der des TMT Pulvers ( $T_{\bmod } 220^{\circ} \mathrm{C}, p_{\bmod } 5$ bar) (rot) und des TMT Pulvers in Toluol (schwarz). Die ESR Messungen wurden bei $60^{\circ} \mathrm{C} 80$ Minuten durchgeführt.

Diese Ergebnisse bestätigen die Aussage, dass die Stabilität der freien Radikalen im TMT durch begrenzte Beweglichkeit der Substituenten und durch den delokalisierten Elektronenzustand in der konjugierten Ligninstruktur vorkommt (s. Abs. 2.2.2). 


\subsection{Fazit}

In diesem Kapitel wurde bewiesen, dass sich die freien Radikale im TMT mittels einer radikalischen Polymerisation mit Styrol gewinnbringend einsetzen lassen. Dafür wurde eine erfolgreiche radikalische Polymerisation in der Substanz ohne den Initiator durchgeführt. Die zahlreichen Analysemethoden bestätigen, dass die Proben mit der höheren Radikalkonzentration, so wie modifizierte Buche mit $T_{\text {mod }} 200^{\circ} \mathrm{C}$ und $220^{\circ} \mathrm{C}$ oder modifizierte Kiefer mit $T_{\bmod } 220^{\circ} \mathrm{C}$, gut dafür geeignet sind. So zeigt die TGA-Analyse zwei TG-Stufen für diese Proben. Die erste TG-Stufe befindet sich in dem Temperaturbereich zwischen $225^{\circ} \mathrm{C}$ und $385^{\circ} \mathrm{C}$, das dem Bereich der Holzzersetzung entspricht. Die zweite TG-Stufe liegt zwischen $385^{\circ} \mathrm{C}$ und $685^{\circ} \mathrm{C}$ und entspricht dem Bereich des Abbaus des Polystyrols. Auch die IR Spektren bestätigen die These - die freien Radikale aus TMT sind aktiv trotz ihrer Stabilität und nehmen in der radikalischen Polymerisationsreaktion ohne den Initiator teil - und zeigen ganz deutlich die IR Banden des Polystyrols mit den Holzproben, die eine hohe Radikalkonzentration haben. Proben mit der niedrigen Radikalkonzentration zeigen keine Anwesenheit vom Polymer. Vermutlich ist die Menge des Polystyrols so klein, dass sie durch diese Methoden nicht möglich ist festzustellen. Dafür spricht auch die verbesserte Hydrophobizität von allen Proben. Auch von denen, die keinen Polystyrol laut IR- und TGA-Analysen enthalten. WCA Messungen zeigen, dass auch nicht modifizierte Kiefer, die mit St behandelt wurde, gute hydrophobe Eigenschaften hat.

Die Blindversuche der radikalischen Polymerisation in der Lösung mit unterschiedlichen Monomeren zeigen negative Ergebnisse. Daraus lässt sich sagen, dass die freien Radikalen aus TMT in einer gelösten Form aufgrund der erhöhten Beweglichkeit miteinander abreagieren und ohne den Initiator keine radikalische Polymerisationsreaktion möglich ist. Das bestätigt die These, dass die Stabilität der freien Radikalen durch eine begrenzte Mobilität im Lignin erklärt wird.

Anschließend wurden die ESR Ergebnisse präsentiert, die während der radikalischen Polymerisation direkt in einem ESR-Röhrchen bei $60{ }^{\circ} \mathrm{C}$ drei Stunden permanent gemessen wurden. Es wurde gezeigt, wie die Radikalkonzentration in TMT während der Polymerisationsreaktion in der ersten Stunde sinkt und die nächsten zwei Stunden relativ stabil bleibt. 
Diese Ergebnisse beweisen endgültig, dass sich die freien Radikale in TMT gewinnbringend einsetzen lassen. Es ist möglich diese Radikale in der radikalischen Polymerisationsreaktion nützlich $\mathrm{zu}$ machen und in der Reaktion direkt, ohne den Initiierungsschritt anzuwenden. 



\section{Kapitel 5 Schlussbemerkungen und Ausblick}

In dieser Arbeit wurden Laub- und Nadelholz thermisch modifiziert und die Auswirkung der Hitze auf die Holzstruktur genauso wie die hitzeinduzierte Radikalbildung mit Hilfe von unterschiedlichen Analysemethoden erfolgreich untersucht.

Im ersten Teil wurden thermisch modifiziertes $\mathrm{Holz}$ und isolierte Holzbestandteile wie das Lignin, die Cellulose und die Hemicellulose (Xylose) separat charakterisiert und mit einander verglichen. Die TGA-Analyse hat signifikante Unterschiede zwischen den Zersetzungsprozessen im Holz und in den separat isolierten Holzbestandteile gezeigt. Dies betont die Komplexität des Themas und erklärt die widersprüchlichen Ergebnisse in der Literatur und aus der Praxis. Diese Differenzen wurden durch die synergistischen Abbaureaktionen der Komponenten erklärt. Konkurrierende Prozesse starten unter der Temperaturerhöhung und beeinflussen sich gegenseitig.

Ebenfalls haben die TGA-Ergebnisse eine höhere thermische Stabilität des thermisch modifizierten Holzes bewiesen. Dabei wurde festgestellt, dass die thermische Stabilität direkt proportional zu der Modifikationsstufe ist. Diese Eigenschaft des TMT hat ein großes Potential für die Holzindustrie und sollte weiter untersucht werden.

Die Änderung des Verhältnisses der Holzkomponenten im thermisch modifizierten Holz mit unterschiedlichen Modifikationsparametern wurde ebenfalls untersucht. Die Ergebnisse haben gezeigt, mit der steigenden Modifikationstemperatur steigt der Lignin-Anteil in beiden Holzarten, sowohl in der Buche als auch in der Kiefer. Dies wurde durch die unterschiedliche Geschwindigkeit in den Zersetzungsprozessen des Lignins, der Cellulose und der Hemicellulose mit Hilfe von den TGAErgebnissen deutlich erklärt. Unerwartet waren die Ergebnisse für die thermisch modifizierte Kiefer in dem Modifikationstemperaturbereich zwischen $200^{\circ} \mathrm{C}$ und $220^{\circ} \mathrm{C}$, wobei der Lignin-Anteil bei $220^{\circ} \mathrm{C}$ niedriger ist als bei $200^{\circ} \mathrm{C}$. Im Gegensatz dazu haben die Holocellulose-Anteile der Kiefer ein entsprechend umgekehrtes Bild gezeigt und somit die Ergebnisse für das Lignin unterstützt. Für die thermisch modifizierte 
Buche wurde dieser Effekt praktisch nicht mehr beobachtet. Daraus folgt, dass das Lignin in der Kiefer während der thermischen Modifizierung im Temperaturbereich zwischen $200^{\circ} \mathrm{C}$ und $220^{\circ} \mathrm{C}$ sich schneller zersetzt als in der Buche im gleichen Temperaturbereich. Die TGA-Ergebnisse und die Bestimmung des Massenverlustes nach der thermischen Modifizierung haben diese Aussage ebenfalls unterstütz.

Die verbesserte Hydrophobizität des Holzes durch die thermische Modifizierung wurde ebenfalls untersucht. Der hydrophobe Charakter des untersuchten Materials ist von der Modifizierungsstufe abhängig. Dies wurde durch den hitzeinduzierten Abbau der Hemicellulose erklärt, die sehr hydrophil ist und Wasser im Holz speichert.

Die Bildung der freien Radikalen im TMT war der Mittelpunkt dieser Arbeit und wurde mit der ESR-Analyse durchgeführt. Die Abhängigkeit der ESR-Signalintensität der Radikalen wurde von den Modifikationsparametern, der Holzart, der relativen Luftfeuchtigkeit und von der Zeit nach der thermischen Modifikation untersucht. Eine enge Korrelation zwischen der Modifikationsparametern und der Radikaldichte im TMT wurde bestätigt. Eine intensive Radikaldichte wurde im Temperaturbereich ab $220{ }^{\circ} \mathrm{C}$ beobachtet. Gerade in diesem Temperaturbereich fängt sich das Lignin abzubauen. Dies bestätigt die These, dass sich die freien Radikalen im TMT am meisten aus dem Lignin bilden.

Signifikante Unterschiede zwischen der Buche und der Kiefer wurden in dem Temperaturbereich ab $200{ }^{\circ} \mathrm{C}$ und höher beobachtet. Wesentlich mehr freie stabile Radikale bilden sich im Laubholz als im Nadelholz. Dies liegt vermutlich am höheren Anteil der $\beta$-O-4-Bindungen und der $\mathrm{OCH}_{3}$ Gruppen im Laubholz-Lignin als im Nadelholz-Lignin. Diese brechen unter der Hitze und bilden die freien stabilen Radikalen.

Die Stabilität der freien Radikalen wurde durch den delokalisierten Elektronenzustand in der konjugierten Lignin-Struktur und durch die begrenzte Beweglichkeit der Substituenten erklärt.

Im zweiten Teil wurde das Lignin als einzelne Holzkomponente thermisch untersucht, um das komplexe Holz-System zu vereinfachen und die Zersetzungsmechanismen leichter $\mathrm{zu}$ interpretieren. Dafür wurden drei Lignin-Arten (das technische Lignin (Indulin, Organosolv), das extrahierte aus der TM Buche und der TM Kiefer und das aus dem Coniferylalkohol synthetisierte Lignin Modell) thermisch behandelt, mit zahlreichen Analysemethoden charakterisiert und mit den Referenzproben verglichen. 
Die NMR und IR Analysen haben sehr informative Ergebnisse geliefert. Mit dem Austieg der Behandlungstemperatur sinkt der Anteil der $\mathrm{OCH}_{3}-$ Gruppen im Indulin. Bis $150{ }^{\circ} \mathrm{C}$ passiert es sehr langsam, aber von $150{ }^{\circ} \mathrm{C}$ bis $200^{\circ} \mathrm{C}$ wurde der Anteil der $\mathrm{OCH}_{3}-G r u p p e n$ um die Hälfte weniger als bei der Referenzprobe. Die $\beta$-O-4-Bindungen waren bis $150{ }^{\circ} \mathrm{C}$ im Indulin sehr stabil, aber bei $200{ }^{\circ} \mathrm{C}$ wurden diese Bindungen fast komplett gebrochen. Im Organosolv haben die $\beta$-O-4-Bindungen gefehlt, weil sie schon während der Gewinnungsprozesse gebrochen wurden. Dafür wurden die ${ }^{1} \mathrm{H}-\mathrm{NMR}$-Signale von Protonen aus dem Zimtaldehyd und der phenolischen $\mathrm{OH}-G r u p p e n$ bei $200{ }^{\circ} \mathrm{C}$ im Organosolv fast nicht mehr erkennbar.

Für das Lignin Modell haben die NMR-Spektren bestätigt, dass die Synthese aus dem Coniferylalkohol erfolgreich war und die Struktur des Lignin Modells sehr nah zur Struktur des Indulins liegt. Unter der Hitze wurden auch hier die $\mathrm{OCH}_{3}-G r u p p e n$, die phenolische und die alifatische $\mathrm{OH}-\mathrm{Gruppen}$ abgebaut. Die $\beta$-O-4-Bindungen waren ähnlich wie im Lignin bis $150{ }^{\circ} \mathrm{C}$ stabil und danach wurden sie komplett zersetzt.

Unter der Hitzeeinwirkung bilden sich neue intramolekulare Verknüpfungen. Die zahlreichen Analysemethoden haben diese These bestätigt. Dadurch wurden die Molmasse und der Vernetzungsgrad größer als in der Referenzprobe.

Die Steigerung des Massenverlusts des Lignins unter der Hitze wurde bestätigt. Unerwartet war, dass der Massenverlust vom Indulin höher als vom Organosolv war, obwohl im thermisch modifizierten Holz der Trend in die Gegenrichtung verläuft und der Massenverlust von der Buche größer ist als von der Kiefer. Der Unterschied wurde durch die Zersetzungsprozesse von anderen Holzkomponenten erklärt. Diese Ergebnisse unterstützen ebenfalls die These, dass das Lignin aus der Kiefer sich schneller abbaut als das Lignin aus der Buche.

Im letzten dritten Teil dieser Arbeit wurde bewiesen, dass sich die freien Radikalen im TMT mittels einer radikalischen Polymerisation mit Styrol gewinnbringend einsetzen lassen. Eine radikalische Polymerisation wurde in der Substanz ohne den Initiator erfolgreich durchgeführt. Die zahlreichen Analysemethoden haben gezeigt, dass die Proben mit der höheren Radikalkonzentration eine Menge vom Polymer enthalten. Proben mit der niedrigen Radikalkonzentration haben keine Anwesenheit vom Polymer gezeigt. Vermutlich war die Menge des Polystyrols so klein, dass sie durch diese Methoden nicht identifiziert wurden. 
Zum Schluss wurden die ESR-Ergebnisse vorgestellt, die direkt während der radikalischen Polymerisation in einem ESR-Röhrchen bei $60^{\circ} \mathrm{C}$ drei Stunden lang durchgeführt wurden. Die Radikalkonzentration im TMT ist in der ersten Stunde gesunken und in den nächsten zwei Stunden war sie relativ stabil. Daraus folgt, dass gerade diese freien Radikalen aus dem TMT in der radikalischen Polymerisationsreaktion eingesetzt wurden. Dies ermöglicht, die radikalische Polymerisationsreaktion um einen Initiierungsschritt zu verkürzen und keinen Initiator anzuwenden. 


\section{Kapitel 6 Experimenteller Teil}

\subsection{Geräte und Analysemethoden}

Im experimentellen Teil werden die verwendeten Geräte und die Analysemethoden genauer beschrieben.

\subsubsection{Gelpermeationschromatographie (GPC)}

Die Molmassenverteilung wurde mithilfe von GPC bestimmt. Die GPC-Anlage der Firma AGILENT TECHNOLOGY vom Typ 1260 INFINITY bestand aus einer HPLC-Pumpe (G1310B, 1260 ISOPUMP); einem Autosampler (G1329B, 1260 ALS), einer Säulenkolonne, welche sich aus einer Vorsäule (PSS SDV, $8 \times 50 \mathrm{~mm}$, Partikelgröße $5 \mu \mathrm{m}$ ) und drei Trennsäusel (PSS SDV, $8 \times 300 \mathrm{~mm}$, Partikelgröße $5 \mu \mathrm{m}$, Porengrößen $10^{6}$, $10^{5}$ und $10^{3} \AA$ ) zusammensetzte, und einem Detektorsystem (RI-Detektor G1314B, 1260 VWDVL; UV-Detektor G1362A, 1260 RID). Als Elutionsmittel wurden THF bei $35^{\circ} \mathrm{C}$ und einer Fließgeschwindigkeit von $1,0 \mathrm{~mL} \mathrm{~min}^{-1}$ und DMAc bei $45^{\circ} \mathrm{C}$ und einer Fließgeschwindigkeit von $0,8 \mathrm{~mL} \mathrm{~min}^{-1}$ verwendet. In beiden Fällen wurde Toluol als interner Standard verwendet und die Eichung erfolgte gegen Polystyrol.

Die Auswertungen wurden mithilfe des Programms WINGPC UNICHROM der Firma PSS gemacht und danach mit dem Programm ORIGINPRO 8.5G graphisch bearbeitet.

\subsubsection{Kernspinresonanz Spektroskopie (NMR)}

Für die Messungen der NMR-Spektren (englisch Nuclear Magnetic Resonance) wurde ein BRUKER AVANCE III HD 500 verwendet. Die chemische Verschiebung $\delta$ ist in ppm gegen TMS angegeben. Die ${ }^{1} \mathrm{H}$-Spektren wurden bei einer Temperatur von $70{ }^{\circ} \mathrm{C}$ und einer Frequenz von $500 \mathrm{MHz}$ aufgenommen. Als Lösungsmittel wurde DMSO- $\mathrm{d}_{6}$ verwendet. Der Lösungsmittelpeak bei $\delta=2,50 \mathrm{ppm}$ diente als interner 
Standard. Für die ${ }^{13} \mathrm{C}$-Spektren wurden Messungen bei $125 \mathrm{MHz}, 70{ }^{\circ} \mathrm{C}$ und mit dem Lösungsmittel DMSO-d 6 über längere Zeit (ca. $11 \mathrm{~h}$ ) aufgenommen. Hierbei diente das Signal des Lösungsmittels bei $\delta=39,520 \mathrm{ppm}$ als interner Standard. Die Konzentration der Substanzen betrug ca. $20 \mathrm{mg} \mathrm{mL}^{-1}$.

Alle Auswertungen der Spektren wurden mithilfe des Programms MESTRENOVA der Firma MESTELAB RESEARCH gemacht.

\subsubsection{Fourier-Transform-Infrarotspektroskopie (FT-IR)}

Zur Messung der FT-IR-Spektren wurden zwei Geräte eingesetzt.

Es wurde einmal FT-IR-Spektrometer der Firma PERKINELMER in der Fakultät Naturwissenschaften und Technik der HAWK Hochschule in der Abteilung der Prof. Dr. Gisela Ohms benutzt. Diese Spektren wurden im Bereich von $400 \mathrm{~cm}^{-1}$ bis $4000 \mathrm{~cm}^{-1}$ und bei einer Auflösung von $8 \mathrm{~cm}^{-1}$ aufgenommen. Jede Probe wurde zehn Mal gescannt. Hierbei ist zu beachten, dass für die pulverartigen Proben eine Methode mit $\mathrm{KBr}$-Pressling ausgesucht wurde. Alle Proben wurden folgendermaßen vorbereitet: $1 \mathrm{mg}$ des Prüfkörpers jeweils mit $299 \mathrm{mg}$ KBr im Mörser fein pulverisiert, danach unter Vakuum mit dem Druck von $10 \mathrm{t}$ zu einer Tablette gepresst und sofort gemessen. Für die Referenzmessung wurde eine Tablette aus reinem $\mathrm{KBr}$ vorbereitet und der Untergrund bestimmt.

Anschließend erfolgten die Auswertungen mit Software derselben Firma. Dabei wurden alle Spektren entsprechend normiert. Nachher wurden Spektren graphisch mithilfe des Programms ORIGINPRO 8.5G bearbeitet.

Für die ATR-IR Messungen wurde ein weiteres FTIR-Spektrometer der Firma BRUKER VERTEX 80v mit den folgenden Einstellungen benutzt: Messbereich zwischen $400 \mathrm{~cm}^{-1}$ und $4000 \mathrm{~cm}^{-1}$; Auflösung $8 \mathrm{~cm}^{-1}$; jeweils 10 Scans; alle Messungen wurden unter Vakuum durchgeführt.

Die Auswertungen wurden mithilfe des Programms OPUS 8.1 gemacht, entsprechend normiert und in ORIGINPRO 8.5G graphisch präsentiert. 


\subsubsection{Elektronenspinresonanz Spektroskopie (ESR)}

Die freien Radikale in den Prüfkörpern wurden mithilfe EPR CW/TRANSIENT Spektrometer System ELEXYS-II 500T der Firma BRUKER BIOSPIN untersucht. Die Spektren wurden mit dem Programm XEPR ebenfalls der Firma BRUKER aufgezeichnet und bearbeitet. Die Einstellungen des ESR-Spektrometers sind in der Tab. 5.1 angegeben.

Tabelle 5.1: Die Einstellungsparameter des ESR-Spektrometers.

\begin{tabular}{|c|c|}
\hline Parameter & Wert \\
\hline Modulationsfrequenz $[\mathrm{kHz}]$ & 100 \\
\hline Modulationsamplitude [G] & 3 \\
\hline Smooth Points & 0 \\
\hline Attenuation [dB] & 23 \\
\hline Frequenz [GHz] & 9,85 (X-Bande) \\
\hline Anzahl der Scans & 10 \\
\hline
\end{tabular}

Anschließend wurden die Auswertungen mit dem Programm ORIGINPRO 8.5G durchgeführt.

\subsubsection{Massenspektrometrie (MS)}

Die Massenspektren der Proben wurden mit einem Elektrospray-Ionisation MAXIS-Massenspektrometer (ESI-QTOF-MS) und einem AUTOFLEX SPEED Massenspektrometer (MALDI-TOF) der Firma BRUKER DALTONIK aufgenommen. Der Messbereich war zwischen 50 und $1650 \mathrm{~m} / \mathrm{z}$. Als Analysator diente in beiden Fällen die Time-of-flight (TOF) Methode. Die Massenspektren wurden mit dem BRUKER COMPASS DATAANALYSIS 4.0 ausgewertet.

\subsubsection{Dynamische Differenzkalorimetrie (DSC)}

Für die Untersuchung des thermischen Verhaltens und für die 
Bestimmung der Glasübergangstemperaturen $T_{\mathrm{g}}$ von den Prüfkörpern wurden DSC-Kurven (englisch Differential Scanning Calorimetry) mithilfe DSC 214 POLYMA der Firma NETZSCH aufgenommen und mit dem Programm PROTEUS THERMAL ANALYSIS der gleichen Firma ausgewertet. Jede Messung wurde im Temperaturbereich zwischen $-30{ }^{\circ} \mathrm{C}$ und $250{ }^{\circ} \mathrm{C}$ mit zwei Zyklen durchgeführt. Die Aufheizrate lag bei $10^{\circ} \mathrm{C} \mathrm{min}^{-1}$.

\subsubsection{Thermogravimetrische Analyse (TGA)}

Die Massenänderung der Proben in Abhängigkeit von der Temperatur und Zeit wurden mithilfe TG 209 F3 TARSUS der Firma NETZSCH untersucht. Der Temperaturbereich wurde zwischen $25^{\circ} \mathrm{C}$ und $1000^{\circ} \mathrm{C}$ mit einer Aufheizrate von $10^{\circ} \mathrm{C} \mathrm{min}{ }^{-1}$ ausgesucht. Alle Messungen wurden unter Stickstoff-Atmosphäre mit der Durchflussrate von $20 \mathrm{~mL} \mathrm{~min}^{-1}$ durchgeführt. Für die Auswertungen wurde das Programm PROTEUS THERMAL ANALYSIS 6.1.0 verwendet.

\subsubsection{Wasserkontaktwinkel (WCA)}

Zur Messung des Wasserkontaktwinkels (englisch Water Contact Angle) wurde DATAPHYSICS OCA 15EC mit einem Messbereich von $0^{\circ}$ bis $180^{\circ}$ verwendet. Das Gerät bestand aus einer VGA-Kamera mit 752 x 480 Pixel und einer LED-Hintergrundbeleuchtung. Alle Messungen wurden bei Raumtemperatur mit demineralisierten Wassertröpfchen mit dem Volumen von $5 \mu \mathrm{L}$ durchgeführt. Vor der WCA-Messung wurden alle Prüfkörper im Ofen bei $103{ }^{\circ} \mathrm{C}$ getrocknet und anschließend wurden die pulverartigen Proben in Tabletten gepresst.

\subsubsection{Dynamische Wasserdampfsorption (DVS)}

Mit Hilfe des DVS-Gerätes (englisch Dynamic Vapor Sorption) SURFACE MEASUREMENT SYSTEMS wurden die Sorption-Isothermen aufgenommen. Dafür wurden die Prüfkörper mit der Masse von 20 mg in einer abgeschlossenen Kammer bei einer konstanten Raumtemperatur von $25{ }^{\circ} \mathrm{C}$ gravimetrisch gemessen. Die relative Luftfeuchtigkeit wurde schrittweise zuerst von $0 \%$ auf $90 \%$ mit einer Rate von $10 \%$ und anschließend auf $95 \%$ erhöht und danach wieder auf $0 \%$ in der 
umgekehrten Reihenfolge abgesenkt. Als die gewünschte relative Luftfeuchtigkeit erreicht wurde, blieb sie konstant, solange die Gewichtsänderung der Probe pro Minute weniger als $0,002 \% \mathrm{~min}^{-1}$ über einen Zeitraum von 10 Minuten wird. Die Soll- und Ist-Messwerte der Massenänderung wurden als Zeitfunktionen aufgetragen und weiter mit dem Programm ORIGINPRO 8.5G ausgewertet.

\subsubsection{Thermische Modifikation des Holzes (TMT)}

Alle Prozesse der thermischen Modifikation des Holzes (englisch Thermally Modified Timber) wurden in einem Behandlungsreaktor im Labormaßstab an der Fakultät für Forstwissenschaften und Waldökologie durchgeführt. Die verwendete Apparatur war ein Nachbau einer industriellen Anlage für die thermische Holzmodifizierung von Dr. Wilhelmus P. M. Willems und bestand aus einem Edelstahlgefäß mit dem Volumen von 65 L für die Holzproben, einem Kühlsystem, einem Dampfgenerator, einer Vakuumpumpe und einer Gaswaschanlage. Der Temperaturbereich lag zwischen $180^{\circ} \mathrm{C}$ und $220^{\circ} \mathrm{C}$. Zwei Systeme wurden getestet: ein offenes System mit dem normalen Druck und ein geschlossenes System mit dem Druck von 5 bar. Die Modifikationsphase dauerte 3 Stunden und den gesamten Prozessen zwischen 20 und 30 Stunden. Alle Prozesse wurden unter Wasserdampfatmosphäre durchgeführt. 


\subsection{Verwendete Chemikalien und Materialien}

\subsubsection{Holzmaterialien}

Proben aus Kiefer und Buche wurden von der Georg-August-Universität der Fakultät für Forstwissenschaften und Waldökologie in Göttingen zur Verfügung vorgelegt. Holzmaterialien wurden entsprechend präpariert (s. Abs. 6.3.1) und im Ofen bei $103^{\circ} \mathrm{C}$ vor jeder Anwendung getrocknet.

\subsubsection{Lignin}

Zwei Arten von technischem Lignin wurden verwendet. INDULIN AT WESTVACO, USA Lignin auch als Kraft-Lignin bekannt, wurde von Prof. Dr. Carsten Mai und Dr. Marco Fleckenstein aus der Fakultät für Forstwissenschaften und Waldökologie in Göttingen gestellt. Dieses Lignin ist eine Mischung mit dem größten Anteil aus Kiefer. Und ORGANOSOLV Lignin, welches aus Buche gewonnen wurde und freundlicherweise von Herrn Gerd Unkelbach aus Fraunhofer-Zentrum für ChemischBiotechnologische Prozesse CBP aus Leuna zur Verfügung gestellt wurde.

\subsubsection{Monomere}

Methylacrylat (MA, 99\%, SIGMA ALDRICH, stabilisiert mit ca. 15 ppm 4-methoxyphenol), Styrol (St, 99\%, SIGMA ALDRICH) und Na-4-Vinylbenzolsulfonat (NaStS, 99\%, SIGMA ALDRICH) wurden verwendet. Inhibitor aus flüssigem Monomeren wurden vor der Verwendung durch säulenchromatographische Aufreinigung über inhibitor remover (SIGMA ALDRICH) entfernt. Monomere wurden immer frisch vorbereitet. 


\subsubsection{Andere Chemikalien}

Aceton (VWR CHEMIKALIEN), Coniferylalkohol (CoAl, 98\%, SIGMA ALDRICH), Dimethylsulfoxid (DMSO, SIGMA ALDRICH), Essigsäure (SIGMA ALDRICH), Natriumhydroxid (NaOH, SIGMA ALDRICH), Natriumchlorid $(\mathrm{NaCl}$, SIGMA ALDRICH), Peroxidase (horseradish, THRMOFISHER), Toluol (SIGMA ALDRICH), Wasserstoffperoxid Lösung ( $\mathrm{H}_{2} \mathrm{O}_{2}$-Lösung, ca. 35\%, SIGMA ALDRICH).

\subsection{Experimentelle Vorgehensweisen}

\subsubsection{Probenpräparation für TMT}

Alle Holzproben wurden von der Fakultät für Forstwissenschaften und Waldökologie zur Verfügung vorgelegt. Die Prüfkörper wurden mit der Größe $20 \times 50 \times 650 \mathrm{~mm}^{3}$ (radial $\times$ tangential $\times$ longitudinal) aus der Kiefer und Buche hergestellt. Um die Risse und andere negative Effekte während der Modifikation zu minimieren, wurden die Proben mit dem durchschnittlichen Feuchtigkeitsgehalt von $12 \%$ für die thermischen Modifikationsprozesse verwendet.

\subsubsection{Probenpräparation für ESR Messung}

Für die ESR Messungen wurden feste Proben in Form von Pulver oder Holzblock und auch flüssige Proben präpariert.

Proben aus dem Holzblock wurden in Zylinderform mit der Länge $5 \mathrm{~mm}$ und dem Durchmesser $3 \mathrm{~mm}$ herausgedrückt und in ESR-Röhrchen platziert.

Für die pulverartigen Proben wurde eine bestimmte Menge direkt in ESRRöhrchen eingewogen.

Flüssige Proben wurden vorher von Sauerstoff durch drei freeze-pump-andthaw-Zyklen an einer Hochvakuumpumpe entgast und in einer Glovebox in ESR-Röhrchen gefüllt. 


\subsubsection{Thermische Modifikation des Holzes (TMT)}

Alle Prozesse wurden mit dem Dr. Maximilian Wentzel Vietheer von der Fakultät für Forstwissenschaften und Waldökologie durchgeführt.

Für die thermische Modifikation wurden zwei Holzarten (Buche als Laubholz und Kiefer als Nadelholz) ausgesucht und präpariert. Jeder Prozess bestand aus drei Phasen. In der ersten Phase wurde Holz getrocknet und langsam erhitzt. Für ein offenes System mit dem Druck $p=1 \mathrm{~atm}$. wurde ein Verfahren ${ }^{111}$ mit der folgenden Aufheizungsrate gewählt: $12{ }^{\circ} \mathrm{Ch}^{-1}$ bis $100{ }^{\circ} \mathrm{C} ; 2^{\circ} \mathrm{C} \mathrm{h}^{-1}$ von $100^{\circ} \mathrm{C}$ bis $130{ }^{\circ} \mathrm{C}$ und wieder $12^{\circ} \mathrm{C} \mathrm{h}^{-1}$ von $130^{\circ} \mathrm{C}$ bis zur Modifikationstemperatur $T_{\text {mod. }}$. Die Zweite Phase war die Modifikationsphase bei der gewünschten $T$ mod. $\left(180{ }^{\circ} \mathrm{C}\right.$, $200{ }^{\circ} \mathrm{C}$ oder $220^{\circ} \mathrm{C}$ ) und dauerte drei Stunden. Während der dritten Phase sank die Temperatur mit einer Geschwindigkeit von $20^{\circ} \mathrm{C} \mathrm{h}^{-1}$, bis $65^{\circ} \mathrm{C}$ erreicht wurde.

Für ein geschlossenes System mit dem Druck $p$ bis 5 bar wurde das WTT-Verfahren ${ }^{112}$ verwendet. Am Anfang wurde ein Vorvakuum (ca. $13 \mathrm{kPa}$ ) angelegt, und erst dann wurde die Temperatur langsam um $12{ }^{\circ} \mathrm{C}$ pro Stunde erhöht. Als die Modifikationstemperatur erreicht wurde, startete die Modifikationsphase wieder für drei Stunden. Zum Schluss wurden die Holzproben mit der Temperaturrate von $20^{\circ} \mathrm{C}$ pro Stunde bis $65^{\circ} \mathrm{C}$ abgekühlt.

Die Zeit für die geschlossenen Prozesse war fast $30 \%$ kürzer als für die offenen Prozesse und lag zwischen 19 und 21 Stunden.

\subsubsection{Massenverlust der Holzproben (ML)}

Zur Bestimmung des durch die thermische Modifikation verursachten Massenverlustes (englisch Mass Loss) ist die Gleichung 6.1 angewendet,

$$
\mathrm{ML}=\frac{\left(m_{\mathrm{BM}}-m_{\mathrm{AM}}\right)}{m_{\mathrm{BM}}} \cdot 100 \%
$$

wobei $m_{\mathrm{BM}}$ die Masse der trockenen Holzprobe vor der thermischen Modifikation ist und $m_{\mathrm{AM}}$ die Masse der trockenen Holzprobe nach der thermischen Modifikation. 


\subsubsection{Bestimmung der chemischen Zusammensetzung von TMT}

Um die chemische Zusammensetzung des thermisch modifizierten Holzes zu bestimmen, wurden Methoden verwendet, die ursprünglich für nicht modifiziertes Holz beabsichtigt waren, aber, wie Experimente von Wentzel et al. gezeigt haben, ${ }^{113}$ diese Verfahren auch für thermisch modifiziertes Holz funktionieren.

Für die Extraktion wurden thermisch modifizierte Buche und Kiefer genommen. Die Modifikationsprozesse sind in dem Abs. 6.3.3 beschrieben. Es wurde ein geschlossenes System mit dem Druck von 5 bar gewählt, um die Dauer der Prozesse zu verkürzen, und die $T_{\text {mod. }}$ von $180^{\circ} \mathrm{C}, 200^{\circ} \mathrm{C}$ und $220^{\circ} \mathrm{C}$, um die Unterschiede im chemischen Molekularniveau $\mathrm{zu}$ untersuchen. Nächste Stufe der Probenvorbereitung war eine Pulverisierung des Holzes bei der niedrigsten Stufe mit Partikelgrößen von $0,2 \mathrm{~mm}$ bis $0,63 \mathrm{~mm}$. Anschließend wurden die Proben im Ofen bei $103^{\circ} \mathrm{C}$ über mehrere Tage getrocknet.

Im nächsten Schritt wurden die Holzproben nach einem Verfahren ${ }^{114}$ der Technical Association of Pulp and Paper Industry (TAPPI) T204 cm-07 von den Extraktstoffen befreit. Dafür wurden $5 \mathrm{~g}$ vom Holzpulver jedes Prozesses in einer Soxhlet-Apparatur mit $150 \mathrm{~mL}$ destillierten Wasser bei einer Temperatur von $100^{\circ} \mathrm{C}$ über 8 Stunden extrahiert. Primär wurde die Extraktion mit einer Mischung aus Ethanol und Cyclohexan (1:2) durchgeführt, aber die Distinktion der Extraktstoffen-Menge liegt in beiden Fällen nur bei 0,2 und 0,7\%, wie in den Experimenten von Altgen et al. gezeigt wurde. ${ }^{115}$ Aus diesem Grund wurde die Extraktion weiter mit dem destillierten Wasser geleitet.

Der Extraktionstoffen-Gehalt ist gravimetrisch nach der Gleichung 6.2 berechnet,

$$
\text { Extraktstoffe }=\frac{\left(m_{1}-m_{2}\right)}{m_{1}} \cdot 100 \%
$$

wobei $m_{1}$ die Masse der trockenen Probe vor der Extraktion ist und $m_{2}$ die Masse der trockenen Probe nach der Extraktion. 
Aus dem extraktfreien Holz wurde Lignin nach Sluiter et al. Methode gewonnen. ${ }^{116}$ Dafür wurden jeweils $300 \mathrm{mg}$ des thermisch modifizierten Holzpulvers aus jedem Modifikationsprozess mit $3 \mathrm{~mL}$ 72-\%iger Schwefelsäure bei $30^{\circ} \mathrm{C}$ verrührt, danach mit demineralisiertem Wasser auf $4 \%$ verdünnt und im Autoklav bei $121^{\circ} \mathrm{C}$ für zwei Stunden platziert. Abfiltrierte und mit Wasser gewaschene Proben wurden im Ofen darrgetrocknet und gravimetrisch bestimmt.

Holocellulose wurde als nächste aus dem thermisch modifizierten und extraktfreien Holzpulver nach Wiese et al. extrahiert. ${ }^{117}$ Dafür wurden $2 \mathrm{~g}$ des Pulvers aus jedem Prozess mit $80 \mathrm{~mL}$ demineralisiertem Wasser, 0,75 g Natriumchlorit und 0,25 mL Essigsäure verrührt und im Autoklav bei der Temperatur von $80^{\circ} \mathrm{C}$ für eine Stunde gelassen. Danach wurde Essigsäure und Natriumchlorit mit den gleichen Mengen wie beim ersten Mal dazugegeben und wieder im Autoklav für eine Stunde gelassen. Dies wurde insgesamt vier Mal wiederholt. Zum Schluss wurde Holocellulose abfiltriert, mit $100 \mathrm{~mL}$ Eiswasser und $25 \mathrm{~mL}$ Aceton gewaschen, im Ofen darrgetrocknet und gravimetrisch bestimmt.

Als letzter Schritt war die Cellulosebestimmung nach TAPPI Test Methode T203 cm-09.118 Hier wurde die Holocellulose mit $75 \mathrm{~mL}$ 17,5-\%iger Natronlauge bei Raumtemperatur gerührt. Nach zwei Stunden wurde die Cellulose abfiltriert und mit $25 \mathrm{~mL}$ 17,5-\%iger Natronlauge, $25 \mathrm{~mL}$ 10-\%iger Essigsäure, $25 \mathrm{~mL}$ Aceton und $150 \mathrm{~mL}$ demineralisiertem Wasser gewaschen. Vor der gravimetrischen Bestimmung wurden die Proben wieder im Ofen getrocknet.

\subsubsection{Synthese eines Ligninmodells aus Coniferylalkohol}

Die Synthese des Lignin Modells aus Coniferylalkohol erfolgte nach Zutropfverfahren von Tanahashi und Higuchi. ${ }^{119}$ Dazu wurde die benötigte Phosphatpufferlösung mit $\mathrm{pH}$-Wert von 6,1 aus den Stammlösungen Kaliumdihydrogenphosphat $\left(1 / 15 \mathrm{~mol} \mathrm{~L}^{-1}\right.$, Stammlösung A) und di-Natriumhydrogenphosphat (1/15 $\mathrm{mol} \mathrm{L}^{-1}$, Stammlösung B) angesetzt. Für $100 \mathrm{~mL}$ der Pufferlösung wurden $15 \mathrm{~mL}$ der Stammlösung B mit $85 \mathrm{~mL}$ der Stammlösung A gerührt. ${ }^{120}$ Insgesamt wurde 1,7 L Pufferlösung benötigt.

Als nächstes wurden drei Lösungen vorbereitet. (I) $1 \mathrm{~g}$ Coniferylalkohol (3,33188 mmmol, Ald rich Chemistry) wurde in 33,33 mL Aceton gelöst und mit 333,33 mL Phosphatpuffer verdünnt. (II) 1,67 mL $\mathrm{H}_{2} \mathrm{O}_{2}$-Lösung (30\%) 
wurden mit 333,33 mL Pufferlösung verdünnt. (III) 16,67 mg Enzym horseradish, Peroxidase (THERMOFISHER (Kandel) $\mathrm{GmbH}$ ) wurden in $1 \mathrm{~L}$ Puffer gelöst. Lösungen (I) und (II) wurden nach dem Zutropfverfahren in der Lösung (III) langsam dazu getropft und bei Raumtemperatur 36 Stunden gerührt. Zum Schluss wurde das Produkt abfiltriert, mit dem destillierten Wasser gewaschen und getrocknet.

\subsubsection{Ligninreinigung}

Die Verunreinigungen in Lignin wurden durch folgendes Verfahren entfernt. ${ }^{121}$ Das Lignin wurde in $1 \mathrm{M} \mathrm{NaOH}$ bei einer Ligninkonzentration von $1 \%$ gelöst und bei Raumtemperatur unter eine Argonatmosphäre 2 Stunden gerührt. Die Lösung wurde zentrifugiert und mit $1 \mathrm{M} \mathrm{NaOH}$ gewaschen. Danach wurde die Lösung mit HCL auf $\mathrm{pH}$-Wert von 2,5 gebracht und erneut zentrifugiert. Der Niederschlag wurde mit $2 \mathrm{M} \mathrm{NaOH}$ bis zu 1-\%iger Ligninkonzentration gelöst und bei $70{ }^{\circ} \mathrm{C} 48 \mathrm{~h}$ unter der Argonatmosphäre gerührt. Mit der Essigsäure wurde die Lösung neutralisiert und in einen Dialyseschlauch übertragen. Die Lösung wurde gegen destilliertes Wasser $48 \mathrm{~h}$ dialysiert, danach mit der Essigsäure auf $\mathrm{pH} 3$ die nächsten $24 \mathrm{~h}$ verdünnt und erneut gegen destilliertes Wasser $24 \mathrm{~h}$ dialysiert. Anschließend wurde das gereinigte Lignin durch Gefriertrocknung gewonnen.

\subsubsection{Thermische Behandlung des Lignins und Bestimmung des Massenverlusts (ML)}

Alle Lignin-Sorten wurden im Ofen bei $150{ }^{\circ} \mathrm{C}, 200^{\circ} \mathrm{C}$ und $220^{\circ} \mathrm{C}$ für $1 \mathrm{~h}$ bzw. $3 \mathrm{~h}$ erhitzt und anschließend der Massenverlust nach der Gleichung 6.3 bestimmt,

$$
\mathrm{ML}=\frac{\left(m_{\mathrm{BML}}-m_{\mathrm{AML}}\right)}{m_{\mathrm{BML}}} \cdot 100 \%
$$

wobei $m_{\mathrm{BML}}$ die Masse des Lignins vor der thermischen Behandlung ist und $m_{\mathrm{AML}}$ die Masse des Lignins nach der thermischen Behandlung. 


\subsubsection{Behandlung des TMT Pulvers mit Styrol durch radikalische Polymerisation in der Substanz}

In dieser elementaren radikalischen Polymerisation von St wurde den durch Initiatoransetzung Radikalbildungsschritt speziell weggelassen, aufgrund der hohen Konzentration der freien Radikalen in TMT.

Vor den Experimenten wurde TMT Pulver erst im Ofen bei $103^{\circ} \mathrm{C}$ ca. 24 Stunden getrocknet. Je Suspension von $150 \mathrm{mg}$ Holzpulver aus thermischen Modifikationsprozessen (Kiefer, Buche, $180^{\circ} \mathrm{C}, 200^{\circ} \mathrm{C}, 220^{\circ} \mathrm{C}$, 5 bar) im 1,5 mL St wurde von Sauerstoff durch die Spülung mit dem Argon 20 Minuten entgast. Anschließend wurde die Polymerisation bei $60^{\circ} \mathrm{C}$ für 3 Stunden durchgeführt. Um die Polymerisationsreaktion abzubrechen, wurde die Polymerisations-mischung mit Luft geflutet und im Eisbad abgekühlt. Nachher wurde TMT Pulver von den PSt-Resten mit dem Toluol gewaschen und getrocknet.

\subsubsection{Behandlung des TMT Pulvers mit Styrol durch elementare radikalische Polymerisation im Lösungsmittel}

TMT Pulver (aus Kiefer und Buche; TMT-Parameter: $180{ }^{\circ} \mathrm{C}$, $200{ }^{\circ} \mathrm{C}$ und $220^{\circ} \mathrm{C}$; 5 bar) wurde $24 \mathrm{~h}$ bei $103{ }^{\circ} \mathrm{C}$ im Ofen vom Wasser befreit. Je $110 \mathrm{mg}$ TMT Pulver wurden in $2 \mathrm{~mL}$ DMSO über längere Zeit gelöst und anschließend mit je 1,1 mL St gerührt. Alle Proben wurden mit dem Argon von Sauerstoff 20 Minuten lang entgast. Die Polymerisationsreaktion wurde bei $60^{\circ} \mathrm{C} 20 \mathrm{~h}$ durchgeführt. Zum Polymerisationsabbruch wurden die Mischungen mit Luft geflutet und im Eisbad abgekühlt. Zum Schluss wurde behandelte TMT Pulver mit dem Toluol jeweils 3 Mal gewaschen und getrocknet.

\subsubsection{Behandlung des TMT Pulvers mit NaStS durch elementare radikalische Polymerisation im Lösungsmittel}

Zuerst wurde TMT Pulver (aus Kiefer und Buche; TMT-Parameter: $180^{\circ} \mathrm{C}$, $200{ }^{\circ} \mathrm{C}$ und $220^{\circ} \mathrm{C}$; 5 bar) im Ofen bei $103^{\circ} \mathrm{C} 24 \mathrm{~h}$ getrocknet. Danach wurden $110 \mathrm{mg}$ TMT Pulver in $2 \mathrm{~mL}$ DMSO lange gelöst und mit $100 \mathrm{mg}$ NaStS gerührt. Zur Sauerstoffbefreiung der Proben wurde Spülung mit dem Argon jeweils 20 Minuten lang verwendet. Die Polymerisationsreaktion wurde bei $75{ }^{\circ} \mathrm{C} 20 \mathrm{~h}$ durchgeführt. Um die Polymerisationsreaktion abzubrechen, wurde die Polymerisations- 
mischung mit Luft geflutet und im Eisbad abgekühlt. Anschließend wurde behandelte TMT Pulver von PNaStS mehrmals mit Wasser ausgewaschen und getrocknet.

\subsubsection{Behandlung des TMT Pulvers mit St durch elementare radikalische Polymerisation in Substanz während ESR- Messung}

Für den Versuch wurde nur thermisch modifizierte pulverisierte Buche mit $T_{\text {mod }} 220^{\circ} \mathrm{C}$ und $p 5$ bar verwendet. Vorher wurde Holz Pulver bei $103{ }^{\circ} \mathrm{C}$ einen Tag lang getrocknet und anschließend mit dem Argon 2 Stunden entgast. Das Monomer wurde durch drei freeze-pump-and-thaw-Zyklen an einer Hochvakuumpumpe (EDWARDS, EXC 120) von Sauerstoff entgast. Das entgaste Monomer sowie das entgaste TMT Pulver wurden in eine Glovebox mit der Argon-Atmosphäre geschleust. Dort wurde eine Probe aus $15 \mathrm{mg}$ TMT Pulver und 0,2 mL St direkt in den ESR-Röhrchen vorbereitet und luftdicht verschlossen.

Während der permanenten ESR-Messungen wurde eine Polymerisationsreaktion bei $60^{\circ} \mathrm{C} 3 \mathrm{~h}$ lang direkt in einem ESR-Röhrchen durchgeführt. Jede 10 Minuten wurde drei Mal die Radikalkonzentration gemessen und anschließend als Funktion gegen Zeit mit dem Programm ORIGINPRO 8.5G aufgetragen. Nach drei Stunden wurde die Reaktion abgebrochen. 
6.1 Geräte und Analysemethoden 


\section{Anhang}

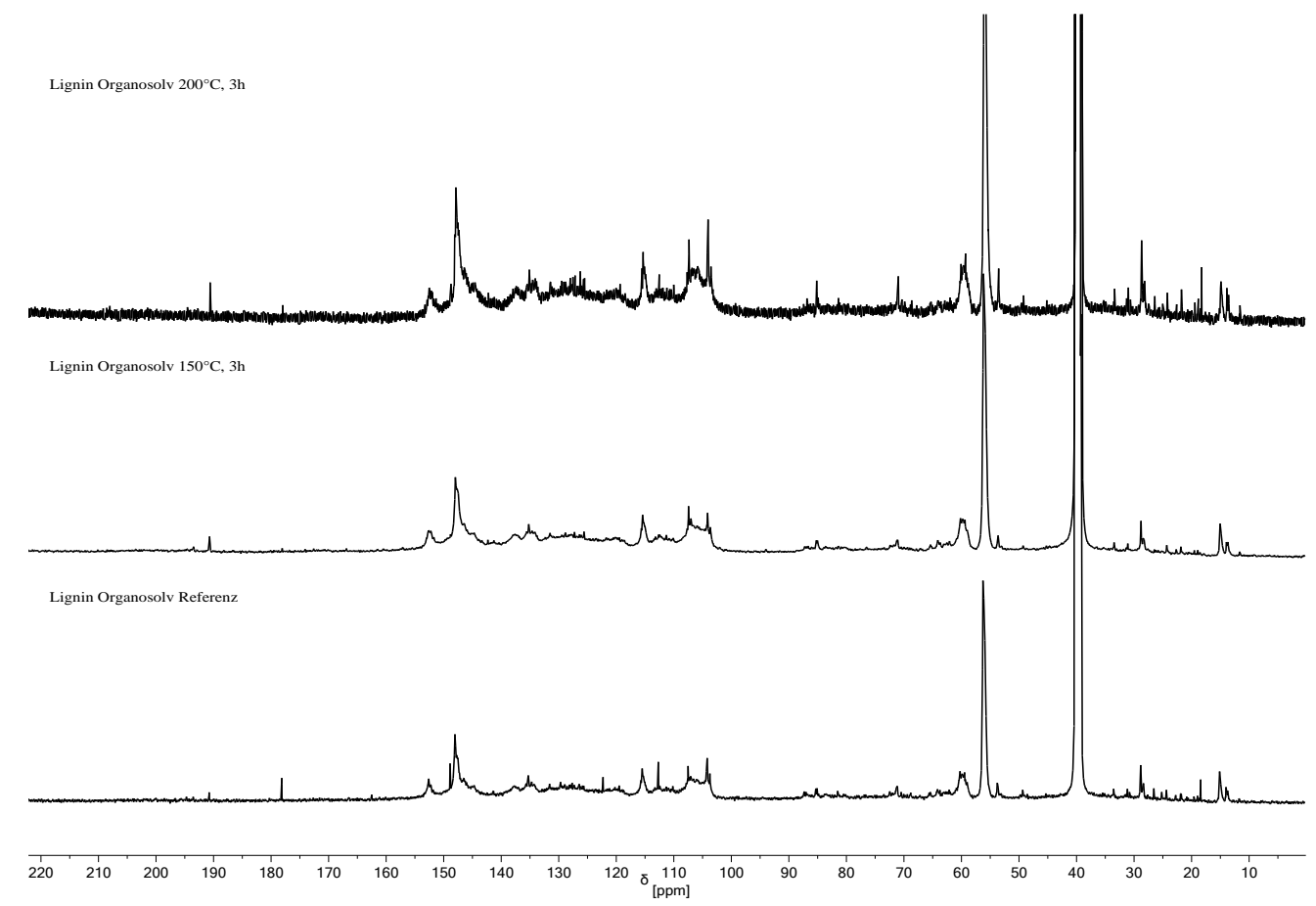

Abb. A: Vergleich der ${ }^{13} \mathrm{C}-\mathrm{NMR}$ Spektren vom thermisch behandelten Organosol Lignin bei $200^{\circ} \mathrm{C}$ (oben), $150^{\circ} \mathrm{C}$ (in der Mitte) und der Referenzprobe (unten). 


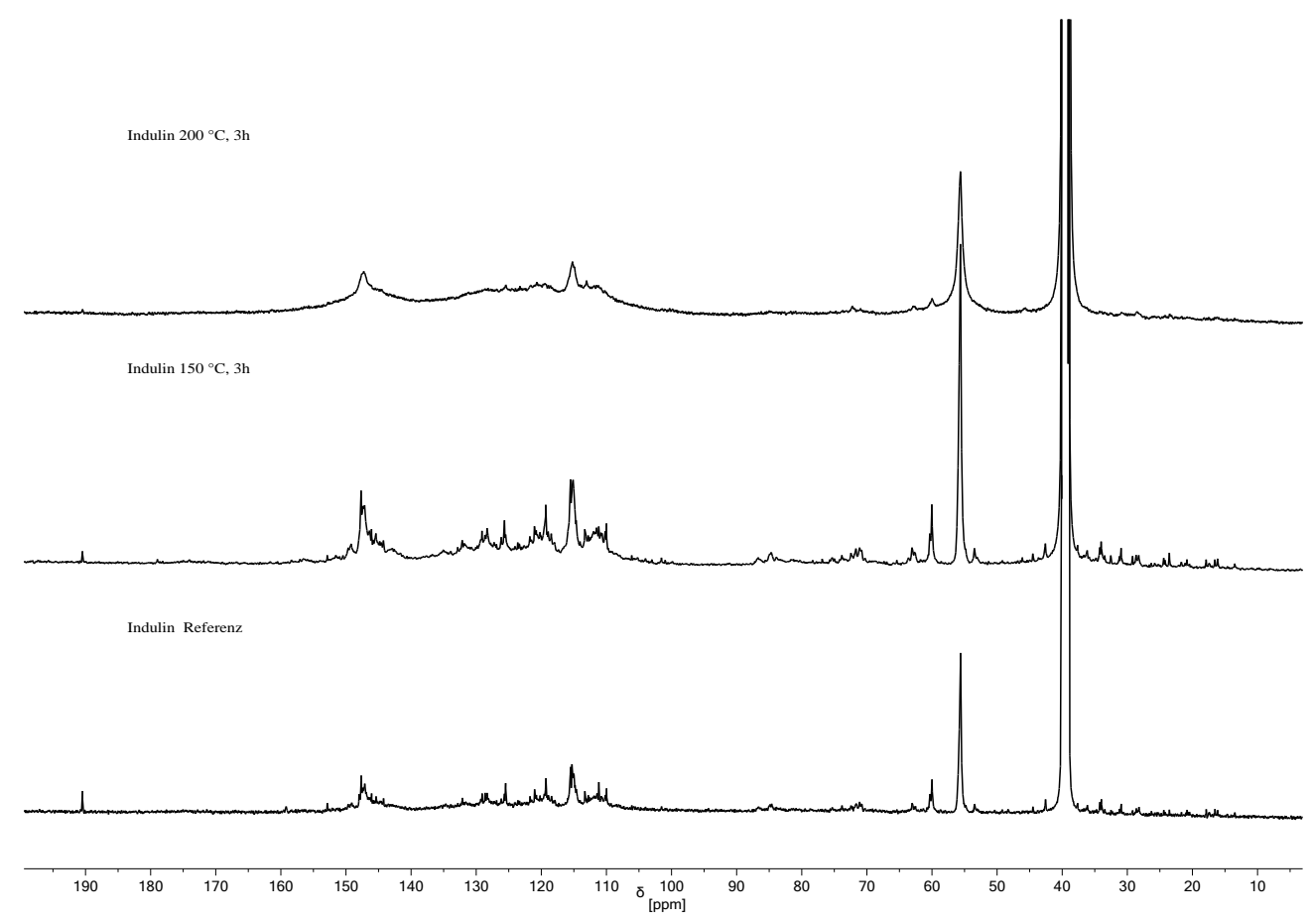

Abb. B: Vergleich der ${ }^{13} \mathrm{C}-\mathrm{NMR}$ Spektren vom thermisch behandelten Indulin Lignin bei $200{ }^{\circ} \mathrm{C}$ (oben), $150{ }^{\circ} \mathrm{C}$ (in der Mitte) und der Referenzprobe (unten).

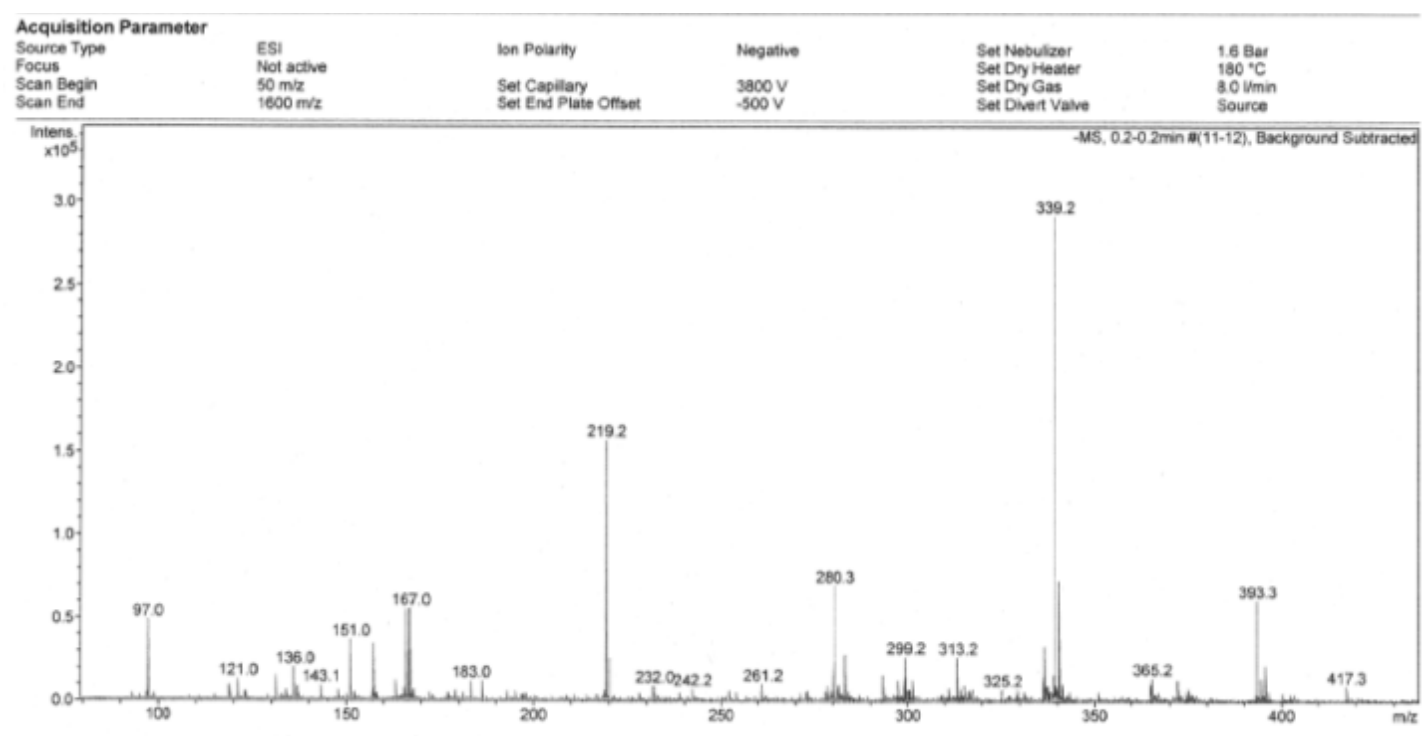

Abb. C: ESI-MS negativ für Indulin Lignin Referenzprobe. 


\section{Abkürzungsverzeichnis}

$$
\%
$$

$\%$ Prozent

$\Delta$

$\Delta \mathrm{B}_{\mathrm{pp}}$ Abstand zwischen den positiven und negativen Peak

0

${ }^{\circ} \mathrm{C}$ Grad Celsius

$$
\mu
$$

$\mu \mathrm{L}$ Mikroliter

A

Abb. Abbildung

Abs. Abschnitt

atm Atmosphere

B

$\mathrm{B}_{0}$ magnetisches Feld am Maximum der Absorptionskurve bzw. beziehungsweise

C

$\mathrm{cm}$ Zentimeter

CML Mittellamelle

Đ

$Ð$ Polydispersität

D

dB Dezibel

DMAc Dimethylacetamid

DMSO Dimethylsulfoxid

DSC Dynamische Differenzkalorimetrie

DVS dynamische Wasserdampsorption 
EMC Gleichgewichtsfeuchtigkeit

$\mathrm{EMC}_{\mathrm{R}}$ reduzierter Gleichgewichtsfeuchtigkeitsgehalt

ESI-QTOF-MS Elektrospray-Ionisation MAXIS-Massenspektrometer ESR Elektronenspinresonanz

$\mathrm{eV}$ Elektronvolt

\section{$\mathbf{F}$}

FT-IR Fourier-Transform-Infrarotspektroskopie

G

g Gramm,

g-Faktor

G Gauß

$\mathrm{GHz}$ Gigahertz

GPC Gelpermeationschromatographie

$\mathbf{H}$

h Stunde, Planck Konstante

I Intensität der Absorptionskurve

IR Infrarot

K

KBr Kaliumbromid

kPa Kilopascal

L

L Liter

M

MALDI Matrix-assisted Laser Desorption/Ionization

mg Milligramm

min Minute

$\mathrm{mL}$ Milliliter

ML Massenverlust, Massenverlust

mm Millimeter 
mol Mol

MS Massenspektroskopie

$\mathrm{NaOH}$ Natriumhydroxid

NaStS Na-4-Vinylbenzolsufonat

NMR Nuclear Magnetic Resonance

\section{$\mathbf{P}$}

P Primärwand

Pa Pascal

$p_{\text {mod }}$ Modifikation Druck

ppm parts per million

PSt Polystyrol

\section{$\mathbf{R}$}

$\mathrm{RH}$ relative humidity

$[\mathrm{R} \bullet]$ Konzentration der freien Radikalen

S Sekundärwand

St Styrol

$t$ Tonnen

Tab. Tabelle

$T$ Anf Anfangszersetzungstemperatur

TAPPI Technical Association of Pulp and Paper Industry

$T_{\mathrm{g}}$ Glasübergangstemperatur

TG Thermogramm

TGA thermogravimetrische Analyse

THF Tetrahydrofuran

TM thermische Modifikation

$T_{\max }$ maximale Temperatur

$T$ mod Modifikation Temperatur

TMT Thermally Modified Timber, Thermally Modified Timber

TOF Time-of-flight 
WCA Wasserkontaktwinkel

$\mathbf{Z}$

z. B. zum Beispiel

$\Delta$

$\delta$ chemische Verschiebung

$\mathbf{M}$

$\mu_{\mathrm{B}}$ Bohr Magneton Moment

$\mathbf{N}$

$v$ Frequenz 


\section{Danksagung}

Zunächst danke ich Herrn Prof. Dr. Philipp Vana dafür, dass er mir die Möglichkeit gegeben hat, das spannende, herausfordernde und vielseitige Thema zu bearbeiten, für sein Vertrauen in meine Fähigkeiten, um eigene Ideen zu entwickeln und kreativ zu sein, und dafür, dass ich meine Promotion in seiner Arbeitsgruppe durchführen durfte, wo ich nicht nur Kollegen aber auch neue Freunde gefunden habe.

Bei Herrn Prof. Dr. Carsten Mai bedanke ich mich für die Übernahme des Korreferats und seine Diskussionsbereitschaft während meiner Promotion. Trotz seines vollen Terminkalenders hatte er Zeit für die Korrekturen und Ratschläge gefunden.

Außerdem möchte ich mich bei meinem Prüfungskomitee bedanken.

Ein besonderer Dank gilt Herrn Prof. Dr. Reinhard Strey von der Universität zu Köln für die motivierende Gespräche und die Unterstützung auch nach meinem Studium.

Für eine äußerst produktive, stressfreie und immer unvergessliche Zeit während der Sommerschulen in Olsberg und in Wernigerode möchte ich mich bei allen Mitgliedern des „MaFoHolz-Programms“ bedanken.

Auch bei meinen fleißigen und motivierenden Bachelorstudentin Lisa Seefried und Bachelorstudent Niklas Klatt möchte ich mich bedanken. Ihre engagierte Arbeit hat zum Erfolg dieser Arbeit beigetragen.

Ein großer Dank gilt Herrn Dr. Maximilian Wentzel Vietheer, der immer ein offenes Ohr für mich hatte und auf dem Gebiet der thermischen Modifikation eine große Hilfe war. In dieser anstrengenden Zeit ist er ein guter Freund geworden.

Bei den ehemaligen Kollegen Herrn Dr. Michael Altgen, Herrn Dr. Wim Willems und Herrn Dr. Marco Fleckenstein möchte ich mich für die zahlreichen Diskussionen und freundliche Hilfe bei den Fragen zum Thema Holz bedanken.

Bei Frau Heike Rohmann, Frau Stefanie Kindler, Frau Sandra Lotze und ehemaligem Mitarbeiter Herrn Dr. Hans-Peter Vögele bedanke ich mich für ihre Hilfe bei praktischen Problemen sowie für die freundliche Atmosphäre und viele lustige Gespräche. 
Zudem möchte ich mich bei Herrn Dr. Tim Koddenberg, Herrn Dr. Gerhard Büttner und Herrn Dr. Markus Hold für die Unterstützung bei administrativen Aufgaben bedanken.

Ein besonderer Dank gilt Herrn Lukas Emmerich für das Messen der dynamischen Wasserdampfsorption, Herrn Ghiath Jnido für die Hilfe bei den IR-Messungen und der Abteilung für Massenspektrometrie des Instituts für Organische und Biomolekulare Chemie für die Aufnahme der Massenspektren.

Für das Korrekturlesen dieser Arbeit danke ich Lara Riemann, Pia Kästner, Stefanie Kindler, Dr. Maximilian Wentzel, Herrn Prof. Dr. Carsten Mai und meinem Ehemann Marco Mühlig.

Bei meinen Freundinnen und Bürokolleginnen Lara Riemann und Katharina Hendrich bedanke ich mich für die sehr gute Arbeitsatmosphäre und die gleichsam inspirierenden und lustigen Momente.

Der Abteilung Vana gilt ein besonders großer Dank für die freundliche Zusammenarbeit, die Hilfsbereitschaft sowie den unvergesslichen Momenten im Theater und in der Oper.

Von ganzen Herzen möchte ich mich bei meiner Familie bedanken, insbesondere bei meinen Eltern Frau Lidia Holstein und Herrn Alexander Holstein, die mich jederzeit während meines Studiums unterstützt haben.

Ein besonders großer Dank gilt meinem verständnisvollen Ehemann Marco! Du warst immer für mich da und hast mir Kraft jeden Tag aufs Neue gegeben! Durch dich sind diese Jahre besonders schön geworden!

Danke! 


\section{Literaturverzeichnis}

${ }^{1}$ C.A.S. Hill, Wood Modifikation. Chemical, Thermal and Other Processes, John Wiley\&Sons, England, 2006

2 T. C. Scheffer, W. E: Eslyn, Effect of heat on the decay resistance of wood, Forest products Journal, 1961

${ }^{3}$ A. J. stamm, H. K. Burr, A. A. Kline, Staybwood-Heat-Stabilized Wood, Industrial and Engineering Chemistry, Vol. 38, 1946

${ }^{4}$ D. Mayers, O. Oksanen, Thermo Wood Handbook. Finnish Thermowood Association, Finland, 2002

${ }^{5}$ W.P. M. Willems, A novel economic large-scale production technology for high quality thermally modified wood. In: F. Engelung, C. A. S. Hill, H. Militz, B. K. Segerholm, The 4th European Conference on Wood Modification, Sweden, 2009

${ }^{6}$ P. Kamdem, A. Pizzi, A. Jermannaud, Durability of heat-treted wood, Holz als Roh- und Werkstoff, Vol. 60, 2002

7 B. F. Tjeerdsma, M. Boonstra, A. Pizzi, P. Tekely, H. Militz, Characterisation of thermally modified wood: molecular reasons for wood performance improvement, Holz als Roh- und Werkstoff, Vol. 56, 1998

8 A. Wagenführ, F. Scholz, Taschenbuch der Holztechnik, Carl Hanser Verlag, München, 2012

9 M. Altgen, C. Welzbacher, M. Humar, W. Willems, H. Militz, Bestimmung der Behandlungsqualität von Thermoholz mithilfe von Schnellverfagren. Teil 1: Elektronenspin-Resonanz-Spektroskopie, Holztechnologie, Vol. 53, 2012

${ }^{10}$ P. K. Kim, J. Kuk, Recent bAdvances in the Processing of wood-Plastic Composites, Springer Berlin, 2010

${ }^{11}$ E. Sjostrom, Wood chemistry: fundamentals and applications, 2. Edition, Academic Press, San Diego, 2013

${ }^{12}$ Y. Li, Advances in Composite Materials - Analysis of Natural and ManMade Materials, Edited by P. Tesinova, InTech, Rijeka, 2011

${ }^{13}$ A. Glazer, H. Nikaido, Microbial Biotechnology - Fundamentals of applied Microbiology, Band 2, Cambridge University Press, 2007

${ }_{14}$ W. Scheiding, P. Grabes, T. Haustein, V. Haustein, N. Nieke, H. Urban, B. Weiß, Holzschutz: Holzkunde - Pilze und Insekten - Konstruktive und chemische Maßnahmen - Technische Regeln - Praxiswissen, Carl Hanser Verlag GmbH Co KG, 2016 
${ }^{15}$ E. Strasburger, Lehrbuch der Botanik, Spektrum Akademischer Verlag Heidelberg, 36. Auflage, 2008

${ }^{16}$ A. Blazej, Chemie des Holzes, VEB Fachbuchverlag Leipzig, 1979

${ }_{17}$ K. Haider, Von der toten organischen Substanz zum Humus, Journal of Plant Nutrition and Soil Science, 162(4), 1998

18 S. J. Eichhorn, A. Dufresne, M. Aranguren, N. E. Marcovich, J. R. Capadona, S. J. Rowan, C. Weder, W. Thielemans, M. Roman, S. Rennecker, W. Gindl, S. Veigel, J. Keckes, H. Yano, K. abe, M. Nogi, A. N. Nakagaito, A. Mangalam, J. Simonsen, A. s. Benight, A. Bismarck, L. A. Berglund, T. Peijs, Journal of Materials Science, Vol. 45, 1-33, 2010

${ }^{19}$ L. J. Gibson, The hierarchical structure and mechanics of plant materials, Journal of the Royal Society Interface, Vol. 9, 2749-2766, 2012

20 D. Fengel, G. Wegener, Wood: chemistry, ultrastructure, reactions, Kessel, München, 2003

${ }^{21}$ O. Faix, Grundlagen der Holzchemie. Vorlesungsskript I, Universität Hamburg, BFH Hamburg, 2004

${ }^{22}$ C. A. S. Hill, Wood Modofication: Chemical, Thermal and Other Processes, Wiley, New York, 2, 2006

${ }^{23}$ A. Glazer, H. Nikaido, Microbial Biotechnology - Fundamentals of applied Microbiology, Band 2, Cambridge University Press, 2007

${ }^{24}$ S. Y. Lin, C. W. Dence, Methods in lignin chemistry, Springer Science \& Business Media, 2012

${ }^{25}$ M. Lewin, I. S. Goldstein, Wood Structures and Composition, Band 11, International Fiber Science and Technology Series, 1991

${ }^{26}$ B. E. Khaldi-Hansen, M. Schulze, B. Kamm, Qualitative and quantitative analysis of lignins from different sources and isolation methods for an application as a biobased chemical resource and polymeric material, Analytical Techniques and Methods for Biomass, Springer, 2016

${ }^{27}$ P. Karlson, D. Doenecke, Karlsons Biochemie und Pathobiochemie, Geotg Thieme Verlag, 2005

${ }^{28}$ A. Tolbert, H. Akinosho, R. Khunsupat, A. K. Naskar, A. J. Ragauskas, Characterization and analysis of the molecular weight of lignin for biorefining studies, Biofpr, Vol. 8,2014

${ }^{29}$ W. E. Hillis, Wood Extractives and Their Significance to the Pulp and Paper Industry, Academic Press, New York, 1962

${ }_{30}$ M. Franck, E.-U. Hartge, S. Heinrich, J. Werther, P. Eidam, I. Fortmann, D. Meier, Gewinnung von Phenolen aus Lignin durch Flash-Pyrolyse 
in einer zirkulierenden Wirbelschicht (ZWS) - Prozessentwicklung und Produktanalytik, DGMK-Tagungsbericht, 2012

${ }^{31}$ S. Kubo, J. F. Kadla, Lignin-based carbon fibers: Effect of synthetic polymer blending on fiber properties, Journal of Polymers and the Environment, Vol 13 (2), 2005

32 A. J. Ragauskas, C. K. Williams, B. H. Davison, G. Britovsek, J. Cairney, C. A. Eckert, W. J. Frederick, J. P. Hallett, D. J. Leak, C. L. Liotta, The path forward for biofuels and biomaterials, Science 311 (5760), 2006

33 J. H. Lora, W. G. Glassrt, Recent industrial applications of lignin: a sustainable alternative to nonrenewable materials, Lournal of Polymers and the Environment, 10(1), 2002

${ }^{34}$ R. Rinaldi, R. Jastrebski, M. T. Clough, J. Ralph, M. Kennema, P. C. Bruijnincx, B. M. Weckhuysen, Wege zur Verwendung von Lignin: Fortschritte in der Biotechnik, der Bioraffination und der Katalyse, Angewandte Chemie, 128(29), 2016 2013

35 E. Sjostrom, Wood chemistry: fundamentals and applications, Elsevier,

${ }^{36}$ H. H. Nimz, M. Roth, U. Tschirner, R. Casten, Organosolv pulping with chloroethanol, Holzforschung, 1986

37 R. El-Hage, N. Brosse, L. Chrusciel, C. Sanchez, . Sannigrahi, A. Ragauskas, Characterization of milled wood lignin and ethanol organosolv lignin from Miscanthus, Polymer Degradation an dStability, Vol. 94, 2009

38 A. J. Stamm, H. K. Burr, A. A. Kline, Heat stabilized Wood (Staywood), Technischer Bericht 1621, Forest Service U. S. Department of Agriculture, 1955

${ }^{39}$ H. D. Tiemann, Effect of different methods of drying on the strength and the hygroscopicity of wood, Vol. 3, Lippincott Co, Philadelphia and London, 1917

${ }^{40}$ E. Giebeler, Dimensional stabilization of wood by moisture-heat-pressuretreatment, Holz Roh Werkst, 41, 1983

41 P. Bekhte, P. Niemz, Effect of high temperature on the change in color, dimensional stability and mechanical properties of spruce wood, Holzforschung, Vol. 57, 2003

42 B. Esteves, H. Pereira, Wood modification by heat treatment, BioRes, Vol. 04, 2009

${ }^{43}$ H. Militz, M. Altgen, Processes and properties of thermally modified wood manufactured in Europe, Deterioration and protection of sustainable biomaterials, Vol. 1158, Oxford University Press, 2014 
${ }^{44}$ D. Mayes, O. Oksanen, ThermoWood Handbook, Finnish Thermowood Association, Helsinki, Finland, 2002

${ }^{45} \mathrm{~W}$. Willems, A novel economic large-scale production technology for high quality thermally modified wood, Proceedings of the $4^{\text {th }}$ European Conference on Wood Modification, Stockholm, Sweden, 2009

${ }^{46} \mathrm{M}$. J. Boonstra, B. Tjeerdsma, H. Groeneveld, Thermal modification of non-durable wood species. Part 1. The Plato thechnology: thermal modification of wood, The International Research Group on Wood Protection Document No IRG/WP 98-40123, 1998

${ }^{47}$ O. Dagbro, P. Torniainen, O. Karlsson, T. Moren, Colour responses from wood, thermally modified in superheated steam and pressurized steam atmospheres, Wood Mater Sci Eng, Vol. 5, 2010

${ }^{48}$ O. Allegretti, M. Brunetti, I. Cuccui, S. Ferrari, M. Nocetti, N. Terziev, Thermo-vacuum modification of spruce (Picea abies Karst.) and fir (Abies alba mill.) wood, BioRes, Vol. 7, 2012

${ }^{49} \mathrm{~W}$. Scheiding, TMT in the year 2018 - an update, Proceedings of the $10^{\text {th }}$ European TMT Workshop, Dresden, Germany, 2018

50 F. Shafizadeh, P. P. S. Chin, Thermal deterioration of wood, Wood Technology: Chemical Aspect, ACS Symposium Series, Vol. 43, 1977

${ }^{51}$ D. Fung, J. Stevenson, J. Shields, The effect of heat and $\mathrm{NH}_{4} \mathrm{H}_{2} \mathrm{PO}_{4}$ on the dimensional and anatomical properties of Douglas-fir, Wood Science, Vol. 7, 1974

${ }^{52} \mathrm{H}$. Rusche, Thermal degradation of wood at temperatures up to $200 \mathrm{deg} C$. Part II. Reaction kinetics of loss of mass during heat treatment of wood, Holz als Roh- und Werkstoff, Vol. 31, 1973

${ }^{53}$ E. Breitmaier, G. Jung, Organische Chemie - Grundlagen, Stoffklassen, Reaktionen, Konzepte, Molekülstruktur, 5. Auflage, Georg Thieme Verlag, Stuttgart, 2005

${ }^{54} \mathrm{H}$. D. Tiemann, The effect of different methods of drying on the strength of wood, Lumber World Review, Vol. 28(7), 1915

${ }_{55}$ F. Shafizaden, P. P. S. Chin, Thermal deterioration of wood, Wood Technology: Chemical Aspects, Vol. 43, 1977

${ }^{56}$ F. Kollmann, E. Schmidt, M. Kufner, D. Fengel, A. Schneider, Gefügeund Eigenschaftsänderungen im Holz durch mechanische und thermische Beanspruchung, Holt Roh Werks, Vol. 27, 1969

${ }^{57}$ F. C. Beall, H. W. Eickner, Thermal degradation of wood components: a review of the literature, Forest Products Laboratory, Forest Service, U.S. 
Department of Agriculture, FPL 130, 1970

${ }^{58}$ C. A. S. Hill, Wood Modification. Chemical, Thermal and Other Processes, Wiley, New York, 2006

${ }^{59}$ J. Bourgois, R. Guyonnet, Characterization and analysis of torrefied wood, Wood Science and Technology, Saint-Etienne, France, 1988

60 B. F. Tjeerdsma, M. Boonstra, A. Pizzi, P. Tekely, H. Militz, Characterisation of thermally modified wood: molecular reasons for wood performance improvement, Holz als Roh- und Werkstoff, Vol. 56, 1998

61 A. Glazer, H. Nikaido, Microbial Biotechnology: Fundamentals of applied Microbiology, Band 2, Cambridge University Press., 2007

62 W. Schneiding, P. Grabes, T. Haustein, V. Haustein, N. Nieke, H. Urban, B. Weiß, Holzschutz: Holzurkunde - Pilze und Insekten - Konstruktive und chemische Maßnahmen - Technische Regeln - Praxiswissen, 2. Auflage, Hanser Verlag, 2016

63 Y. Hao, Y. Pan, R. Du, Y. Wang, Zh. Chen, X. Zhang, X. Wang, The Influence of a Thermal Treatment on the Decay Resistance of Wood via FTIR Analysis, Advances in Materials Science and Engineering, 2018

64 A. A: Shabaka, A. M. A. Nada, Infrared spectroscopic study of thermally treated lignin, Journal of Materials Science, Vol. 25, 1990

${ }^{65}$ R. M. Seborg, H. Tarkow, A. J. Stamm, Effect of heat upon the dimensinal stabilization of wood, Journal of the Forest Products Research Society, Vol. 3, 1953

${ }^{66} \mathrm{H}$. Rusche, Thermal degradation of wood at temperatures up to 200 deg C. Part II. Reaction kinetics of loss of mass during heat treatment of wood, Holz als Roh und Werkstoff, Vol. 31(8), 1973

67 M. A. Millett, G. C. Gerhards, Accelerated aging: residual weight and flexural properties of wood heated in air at $115^{\circ} \mathrm{C}$ to $175^{\circ} \mathrm{C}$, Wood Science, Vol. 4, 1972

${ }^{68}$ F. Gerson, W. Huber, Electron Spin Resonance Spectroscopy of Organic Radicals, Wiley, 2003

69 Ch. Chatgilialoglu, A. Studer, Encyclopedia of Radical in Chemistry, Biology and Materials. Vol. 1: Basic Concepts and Methodologies, Wiley, 2012

${ }^{70}$ G. R. Eaton, S. S. Eaton, D. P. Barr, R. T. Weber, Quantitative EPR, Springer, Wien, 2010

71 M. Altgen, C. Welzbacher, M. Humar, W. Willems, H. Militz, Bestimmung der Behandlungsqualität von Thermoholz mithilfe von Schnellverfahren. Teil 1: Elektronenspin-Resonanz-Spektroskopie, Holztechnologie, Vol. 53, 2012 
${ }^{72}$ R. W. Rex, Electron Paramagnetic Resonance studies of Stable Free Radicals in Lignin and Humic Acids, Nature 188, 1960

${ }^{73}$ S. Barsberg, LG. Thygesen, Spectroscopic properties of oxidation species generated in the lignin of wood fibers by a laccase catalyzed treatment: electronic hole state migration and stabilization in the lignin matrix, Biochim Biophys Acta, 1472(3), 1999

${ }^{74}$ J. K. Kim, K. Pal, Recent Advances in the Processing of Wood-Plastic Composites, Springer, Berlin, Heidelberg, 2010

75 T. Higuchi, Biosynthesis and Biodgeradation of Wood Components, Academic Press, Orlando, Florida, 1985

${ }^{76}$ H.-G. Elias, Makromolekule. Band I: Chemische Struktur und Synthesen, Wiley-VCH, 6 Auflage, 1999

77 R. Grün, Die ESR-Alterbestimmungsmethode, Springer-Verlag, Berlin, 1989

${ }^{78}$ K. Kopitzki, P. Herzog, Einführung in die Festkörperphysik, 5. Auflage, Teubner-Verlag, Stuttgart, 2004

${ }^{79}$ J. K. Kim, K. Pal, Recent Advances in the Processing of Wood-Plastic Composites, Springer, Berlin, Heidelberg, 2010

${ }^{80}$ R. W. Rex, Electron Paramagnetic Resonance studies of Stable Free Radicals in Lignin and Humic Acids, Nature 188, 1960

${ }^{81} \mathrm{~S}$. Barsberg, LG. Thygesen, Spectroscopic properties of oxidation species generated in the lignin of wood fibers by a laccase catalyzed treatment: electronic hole state migration and stabilization in the lignin matrix, Biochim Biophys Acta, 1472(3), 1999

82 A. Sluiter, B. Hames, R. Ruiz, C. Scarlata, J. Sluiter, D. Templeton, D. Crocker, Determination of structural carbohydrates and lignin in biomass, US National Renewable Energy Laboratory, Golden, Colorado, 2008

83 M. Tanahashi, T. Higuchi, Dehydrogenative Polymerization of Monolignols by Peroxidase and $\mathrm{H}_{2} \mathrm{O}_{2}$ in a Dialysis Tube. I. Preparation of Highly Polymerized DHPs, Wood research: bulletin of the Wood Research Institute Kyoto University, Vol. 67, 1981

${ }^{84}$ O. Faix, Classification of Lignins from Different Botanical Origins by FT-IT Spectroscopy, Holzforschung, Vol. 45, 1991

${ }_{85}$ R. El-Hage, N. Brosse, L. Chrusciel, C. Sanchez, . Sannigrahi, A. Ragauskas, Characterization of milled wood lignin and ethanol organosolv lignin from Miscanthus, Polymer Degradation an dStability, Vol. 94, 2009

${ }^{86}$ A. A. Shabaka, A. M. A. Nada, Infrared spectroscopic study of thermally 
treated lignin, Journal of Materials Science, Vol. 25, 1990

87 W. Kubel, Coniferin, ein Glucosid aus dem Cambialsafte der Nadelhölzer, Journal für Praktische Chemie, Vol. 97, 1866

$88 \mathrm{~S}$. Li, K. Lundquist, A new method for the analysis of phenolic groups in lignins by ${ }^{1} \mathrm{H}$ NMR spectrometry, Nordis Pulp \& Paper Research Journal, Vol. 9, 1994

${ }^{89}$ K. Lundquist, NMR Studies of Lignins. 4. Investigation of Spruce Lignin by ${ }^{1} H$ NMR Spectroscopy, Acta Chemica Scandinavica, Vol. 34, 1980

${ }^{90} \mathrm{~K}$. Lundquist, NMR Studies of Lignins. 5. Investigation of Non-derivatized Spruce and Birch Lignin by ${ }^{1} \mathrm{H}$ NMR Spectroscopy, Acta Chemica Scandinavica, Vol. 35, 1981

${ }^{91}$ K. Lundquist, NMR Studies of Lignins. 3. ${ }^{1} \mathrm{H}$ NMR Spectroscopic Data for Lignin Model Compounds, Acta Chemica Scandinavica, Vol. 33, 1979

${ }_{92}$ A.-A. M. A. Nada, M. A. Yousef, K. A. Shaffei, A. M. Salah, Infrared spectroscopy of some treated lignins, Polymer Degradation and Stability, Vol. 62, 1998

93 M. Brebu, C. Vasile, Thermal degradation of lignin, Cellulose Chemistry \& Technology, Vol. 44, 2010

94 M. A. Millett, G. C. Gerhards, Accelerated aging: residual weight and flexural properties of wood heated in air at $115^{\circ} \mathrm{C}$ to $175^{\circ} \mathrm{C}$, Wood Science, Vol. 4, 1972

95 Ch. Chatgilialoglu, A. Studer, Encyclopedia of Radical in Chemistry, Biology and Materials. Vol. 1: Basic Concepts and Methodologies, Wiley, 2012

${ }^{96}$ G. R. Eaton, S. S. Eaton, D. P. Barr, R. T. Weber, Quantitative EPR, Springer, Wien, 2010

97 T. Higuchi, Biosynthesis and Biodgeradation of Wood Components, Academic Press, Orlando, Florida, 1985

98 W. Keim, Kunststoffe. Synthese, Herstellungsverfahren, Apparaturen, Wiley-VCH Verlag, Weinheim, 2006

99 G. Habenicht, Kleben. Grundlagen, Technologien, Anwendungen, 6. Auflage, Springer-Verlag, Berlin, 2009

100 S. Seleem, M. Hopkins, J. Olivio, D. Schiraldi, Comparison of Thermal Decomposition of Polystyrene Products vs. Bio-Based Polymer Aerogels, The Ohio journal of science, 117(2), 2017

101 B. Esteves, A. V. Marques, I. Domingos, H. Pereira, Chemical changes of heat treated pine and eucalypt wood monitored by FTIR, Maderas. Ciencia y tecnologia, Vol. 15, 2013 
102 A.-Y. L.-Bermudez, R. Salazar, Synthesis and characterization of the polystyrene - asphaltene graft copolymer by FT-IR spectroscopy, C.T.F Cienc. Tecnol. Futuro, Vol. 3, Bucaramanga, 2008

${ }^{103}$ S. T. Aruna, P. Binsy, R. Edna, J. B. Bharathibai, Properties of phase separation method synthesized superhydrophobic polystyrene films, Applied Surface Science, Vol. 258, 2012

104 A. Wagenführ, F. Scholz, Taschenbuch der Holztechnik, 2. Auflage, Hanser Verlag, München, 2012

${ }^{105}$ C. Driemeier, F. M. Mendes, M. M. Oliveira, Dynamic vapor sorption and thermoporometry to probe water in celluloses, Cellulose, Vol. 19, 2012

106 C. A. S: Hill, A. J. Norton, G. Newman, The water vapour sorption properties of Sitka spruce determined using a dynamic vapour sorption apparatus, Wood Science and Technology, 2010

107 S. V. Glaass, S. L. Zelinka, Moisture Relation and Physical Properties of Wood, Wood-handbook-Wood as an engineering material, Chapter: 4, 2010

${ }_{108}$ C. M. Popescu, C. A. S. Hill, S. Curling, G. Ormondroyd, Y. Xie, Journal of Materials Science, 49, 2014

${ }^{109}$ Ch. Skaar, Wood-Water Relation, Springer-Verlag, Berlin Heidelberg, 1988

${ }^{110}$ L. Gasparovic, Z. Korenova, L. Jelemensky, Kinetic study of wood chips decomposition by TGA, Chemical Papers, Vol. 65, 2010

${ }^{111}$ D. Mayes, O. Oksanen, ThermoWood Handbook. Finnish Thermowood Association, Helsinki, Finland, 2002

$112 \mathrm{~W}$. Willems, A novel economic large-scale production technology for highquality thermally modified wood. In Proceedings of the $4^{\text {th }}$ European Conference on Wood Modification, Stockholm, Sweden, 2009

113 M. Wentzel, M. Altgen, H. Militz, Analyzing reversible changes in hydroscopicity of thermally modified eucalypt wood from open and closed reactor systems, Wood Science and Technology, Vol. 52, 2018

114 TAPPI, Solvent extractives of wood and pulp. Test method T204 cm-7, Technical Association of the Pulp and Paper Industry Atlanta, Georgia, United States, 1997

${ }^{115}$ M. Altgen, W. Willems, H. Militz, Wood degradation affected by process conditions during thermal modification of European beech in a high-pressure reactor system, European Journal of Wood and Wood Products, Vol. 5, 2016

116 A. Sluiter, B. Hames, R. Ruiz, C. Scarlata, J. Sluiter, D. Templeton, D. Crocker, Determination of structural carbohydrates and lignin in biomass, US 
National Renewable Energy Laboratory, Golden, Colorado, 2008

117 L. E. Wise, M. Murphy, A. A. Daddieco, Chlorite holocelluloses, its fractionation and bearing on summative wood analysis and on studies on the hemicelluloses. Technical Association Papers 29, 1946

118 TAPPI, Alpha-, beta- and gamma-celullose in pulp, Test method T203 cm09, Technical Association of the Pulp ans Paper Industry, Atlanta, Georgia, United States, 2009

119 M. Tanahashi, T. Higuchi, Dehydrogenative Polymerization of Monolignols by Peroxidase and $\mathrm{H}_{2} \mathrm{O}_{2}$ in a Dialysis Tube. I. Preparation of Highly Polymerized DHPs, Wood research: bulletin of the Wood Research Institute Kyoto University, Vol. 67, 1981

120 M. Mulisch, U. Welsch, Romeis-Mikroskopische Technik, Springer Spektrum, 19. Aufl., 2015

${ }^{121}$ S. Y. Lin, C. W. Dence, Methods in Lignin Chemistry, Springer Series in Wood Science, Springer Verlag, Berlin, 1992 